Elsevier Editorial System(tm) for Energy Manuscript Draft

Manuscript Number: EGY-D-19-02887R1

Title: Perturbed stochastic fractal search for solar PV parameter estimation

Article Type: Full length article

Keywords: Photovoltaic (PV) modeling; parameters estimation; stochastic fractal search; chaotic elitist perturbation

Corresponding Author: Dr. Xu Chen,

Corresponding Author's Institution: School of Electrical and Information Engineering, Jiangsu university

First Author: Xu Chen

Order of Authors: Xu Chen; Hong Yue; Kunjie Yu

Abstract: Following the widespread use of solar energy all over the world, the design of high quality photovoltaic (PV) cells has attracted strong research interests. To properly evaluate, control and optimize solar PV systems, it is crucial to establish a reliable and accurate model, which is a challenging task due to the presence of non-linearity and multi-modality in the PV systems. In this work, a new meta-heuristic algorithm (MHA), called perturbed stochastic fractal search (pSFS), is proposed to estimate the PV parameters in an optimization framework. The novelty lies in two aspects: (i) employ its own searching operators, i.e., diffusion and updating, to achieve a balance between the global exploration and the local exploitation; and (ii) incorporate a chaotic elitist perturbation strategy to improve the searching performance. To examine the effectiveness of pSFS, this method is applied to solve three PV estimation problems for different PV models, including single diode, double diode and PV modules. Experimental results and statistical analysis show that the proposed pSFS has improved estimation accuracy and robustness compared with several other algorithms recently developed. 


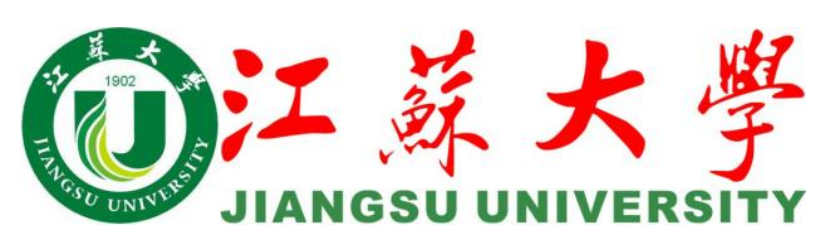

School of Electrical \& Information Engineering Jiangsu University

E-mail address: xuchen@ujs.edu.cn

Dear Editor,

We would like to submit our paper entitled "Perturbed stochastic fractal search for solar PV parameter estimation" for possible publication in Energy. This work is original and has not been published elsewhere nor is it currently under consideration for publication elsewhere.

This paper focuses on modeling the solar photovoltaic (PV) systems based on a new metaheuristic algorithm called perturbed stochastic fractal search algorithm. We think it is suitable for Energy, and would attract wide scientific audience's attention.

We deeply appreciate your consideration of our manuscript, and look forward to receiving comments from the reviewers. Thank you.

Sincerely,

Xu Chen, Hong Yue, Kunjie Yu 


\section{Responses to Reviewers' comments on the manuscript entitled "Perturbed stochastic fractal search for solar PV parameter estimation" (Paper ID: EGY-D-19-02887)}

Dear Professor Kalogirou, dear editor and reviewers,

We appreciate to have this opportunity to revise and improve our manuscript. We have made our best efforts to address all comments and suggestions provided by the Editor and the three reviewers, and have made careful modifications all through the paper. We hope the revised version would satisfy the requirements and standards for the Journal. Thank you.

\section{Response to Reviewer \#1:}

1. Abstracts need to be edited because the necessary material has not been raised.

Our response: Thanks for this suggestion. We have re-written the abstract and added necessary material.

"Following the widespread use of solar energy all over the world, the design of high quality photovoltaic (PV) cells has attracted strong research interests. To properly evaluate, control and optimize solar PV systems, it is crucial to establish a reliable and accurate model, which is a challenging task due to the presence of non-linearity and multi-modality in the PV systems. In this work, a new meta-heuristic algorithm (MHA), called perturbed stochastic fractal search (pSFS), is proposed to estimate the PV parameters in an optimization framework. The novelty lies in two aspects: (i) employ its own searching operators, i.e., diffusion and updating, to achieve a balance between the global exploration and the local exploitation; and (ii) incorporate a chaotic elitist perturbation strategy to improve the searching performance. To examine the effectiveness of pSFS, this method is applied to solve three PV estimation problems for different PV models, including single diode, double diode and PV modules. Experimental results and statistical analysis show that the proposed pSFS has improved estimation accuracy and robustness compared with several other algorithms recently developed."

\section{Explain Figure 5 clearly.}

Our response: Thank you very much for this useful suggestion. To describe more clearly the implementation of the pSFS algorithm, in the revised version, we added the following pseudocode into Appendix 1. We will be happy to move this to the main article if that is helpful.

Appendix 1 Pseudocode of the pSFS algorithm 


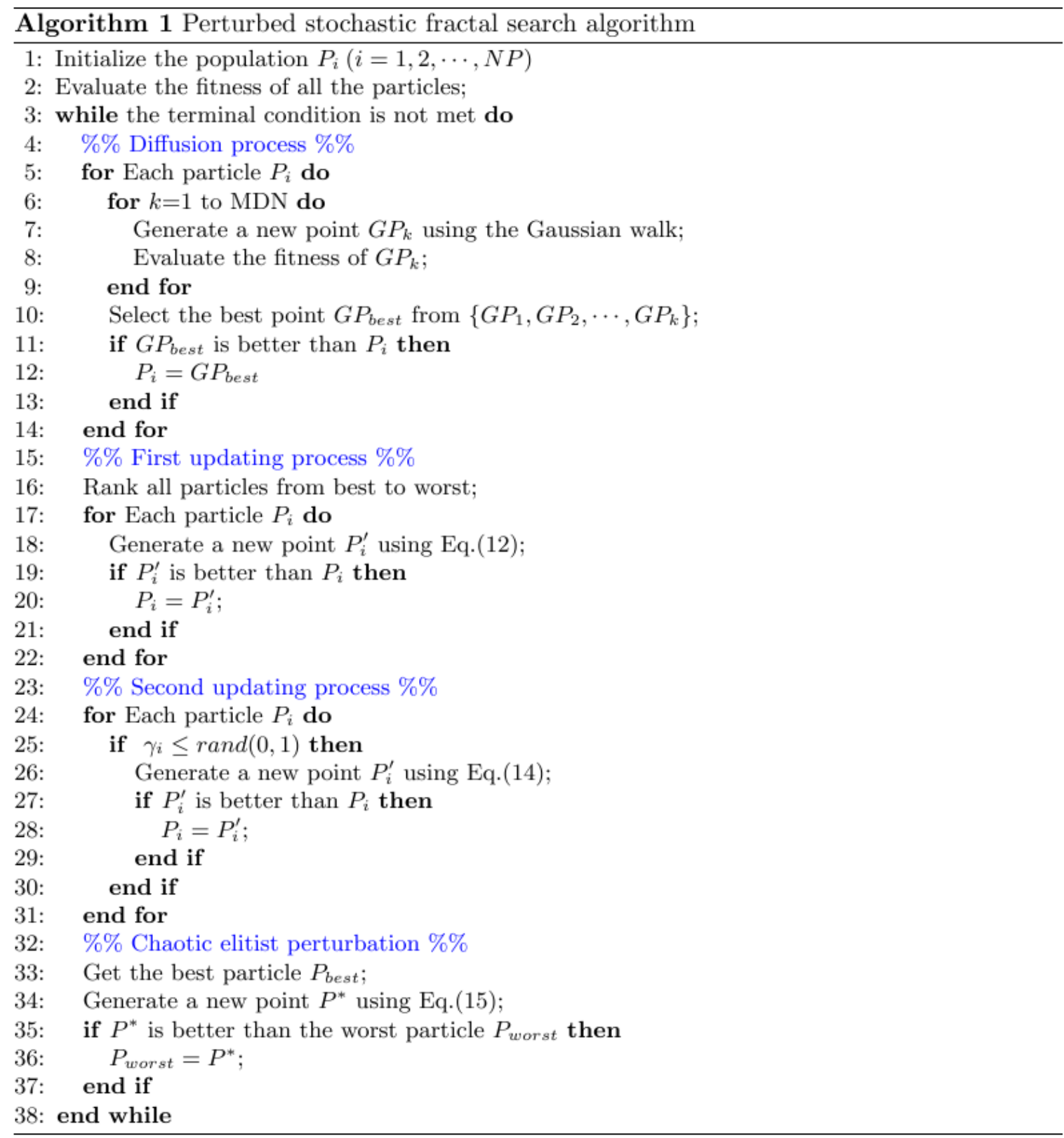

3. The conclusions should present quantitative evaluations of the advantages of the "new method" of control, comparing to the other considered methods.

Our response: Thanks for this comment. We have added quantitative evaluations of the proposed pSFS approach, in particular its high robustness based on standard deviation (SD) and its high accuracy based on Wilcoxon rank sum test.

"The proposed pSFS algorithm is evaluated on PV parameter estimation problems with different diode models. Also, the performance of pSFS is compared with the basic SFS and six non-SFS algorithms. The following conclusions can be drawn from the numerical results.

- $\quad$ pSFS achieves high parameter estimation accuracy for different PV models. The statistical results demonstrate that pSFS has the best results in terms of the optimal, the mean and the worst RMSE values. Moreover, for the 


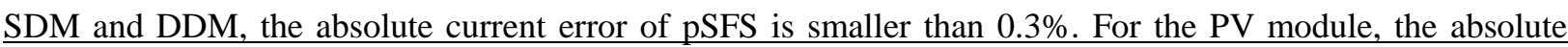
current error of pSFS is smaller than $8 \%$.

- pSFS also has superiority in robustness compared with the recently developed algorithms including SFS, IJAVA, TLABC and GOTLBO. For the SDM, pSFS achieves the smallest SD, the value of which is smaller than 1e-7. For the PV module, pSFS achieves the smallest SD value less than 1e-16.

- $\quad$ pSFS performs better than the other compared algorithms under comparison according to the Wilcoxon rank sum test.

- $\quad$ pSFS has a reasonably fast convergence speed, and keeps a good balance between global exploration and local exploitation during the searching process by employing two search operators.

- $\quad$ PSFS is robust to various environment conditions. The tests on three real PV modules at different irradiance and temperature levels show that pSFS achieves accurate results under all circumstances.

The proposed pSFS gains the above benefits mainly from two aspects. The first is the diffusion and updating processes from the basic SFS, which helps to achieve balance between global and local search. The second is the chaotic elitist perturbation strategy, which further enhances the estimation accuracy and robustness".

4. How do you choose the operating points in case study section?

Our response: Thanks for your comments. The experimental $V$-I data of single diode model (SDM) and double diode model are measured from a RTC France solar cell (under $1000 \mathrm{~W} / \mathrm{m} 2$ at $33{ }^{\circ} \mathrm{C}$ ), and the experimental V-I data of the PV module are from Photowatt-PWP201 module (under 1000 $\mathrm{W} / \mathrm{m} 2$ at $45^{\circ} \mathrm{C}$ ). All the operating points and experimental data are taken from reference [5]:

[5] Easwarakhanthan T, Bottin J, Bouhouch I, Boutrit C. Nonlinear minimization algorithm for determining the solar cell parameters with microcomputers. International Journal of Solar Energy. 1986;4:1-12"

We have added these details to the revised manuscript.

5. The abbreviation is used for the first time whose full form should be mentioned.

Our response: Thank you for this reminder. We have checked the manuscript to make sure that the abbreviation terms are introduced the first time the full form appears in the paper. Once the abbreviations are defined, they are used for the rest of the writing. A nomenclature and abbreviation table is added in the revised manuscript.

\begin{tabular}{|llll|}
\hline Nomenclature & & & \\
$a, a_{1}, a_{2}$ & Diode ideality constant & $k$ & Boltzmann constant $\left(1.3806503 \times 10^{23} \mathrm{~J} / \mathrm{K}\right)$ \\
$D$ & Problem dimension & $N P$ & Population size \\
$F E S$ & Function evaluations & $P_{\text {best }}$ & Position of the best particle \\
$F E S_{\max }$ & Maximum number of function evaluations & $P_{i}$ & Position of the $i$-th particle \\
$G_{\max }$ & Maximum number of generations & $q$ & Electron charge $\left(1.60217646 \times 10^{-19} \mathrm{C}\right)$ \\
$I_{d}, I_{d 1}, I_{d 2}$ & Diode current $(\mu \mathrm{A})$ & $R_{S}$ & Series resistance $(\Omega)$ \\
$I_{L}$ & Output current $(\mathrm{A})$ & $R_{s h}$ & Shunt resistance $(\Omega)$ \\
\hline
\end{tabular}




\begin{tabular}{|llll|}
\hline$I_{p h}$ & Photo-generated current (A) & $T$ & Cell temperature (K) \\
$I_{s d}$ & Reverse saturation current (A) & $V_{L}$ & Cell output voltage (V) \\
$I_{s d 1}$ & Diffusion current (A) & $V_{t}$ & Junction thermal voltage (V) \\
$I_{s d 2}$ & Saturation current (A) & $\mu_{B P}, \mu_{P}$ & Gaussian parameters \\
$I_{s h}$ & shunt resistor current (A) & $\gamma_{i}$ & Selection probability for the $i$-th particle \\
Abbreviations & & & \\
ABC & Artificial bee colony & MDN & Maximum diffusion number \\
BHCS & Biogeography-based heterogeneous cuckoo search & MHA & Meta-heuristic algorithms \\
BLPSO & Biogeography-based learning PSO & pSFS & Perturbed stochastic fractal search \\
CLPSO & Comprehensive learning PSO & P-DE & Penalty based differential evolution \\
DDM & Double diode mode & PSO & Particle swarm optimization \\
DE & Differential evolution & PV & Photovoltaic \\
ELPSO & Enhanced leader PSO & RMSE & Root mean square error \\
FPA & Flower pollination algorithm & SD & Standard deviation \\
FWA & Fireworks algorithm & SDM & Single diode model \\
GOTLBO & Generalized opposition TLBO & SFS & Stochastic fractal search \\
HFAPS & Hybrid firefly algorithm and pattern search & SSA & Salp swarm algorithm \\
HS & Harmony search & TLBO & Teaching-learning-based optimization \\
IACE & Individual absolute current error & TLABC & Teaching-learning-based artificial bee colony \\
IADE & Improved adaptive differential evolution & TRR & Trust-region reflective \\
IAPE & Individual absolute power error & WOA & Whale optimization algorithm \\
\hline
\end{tabular}

\section{Add the measurement unit to the vertical axis of some of figures}

Our response: Thank you for the good observation. We have checked all the figures, and found the measurement units are lost for the absolute current and power error in Fig.7, Fig.9 and Fig.11. We have added the units (A) and (W) in the legend.

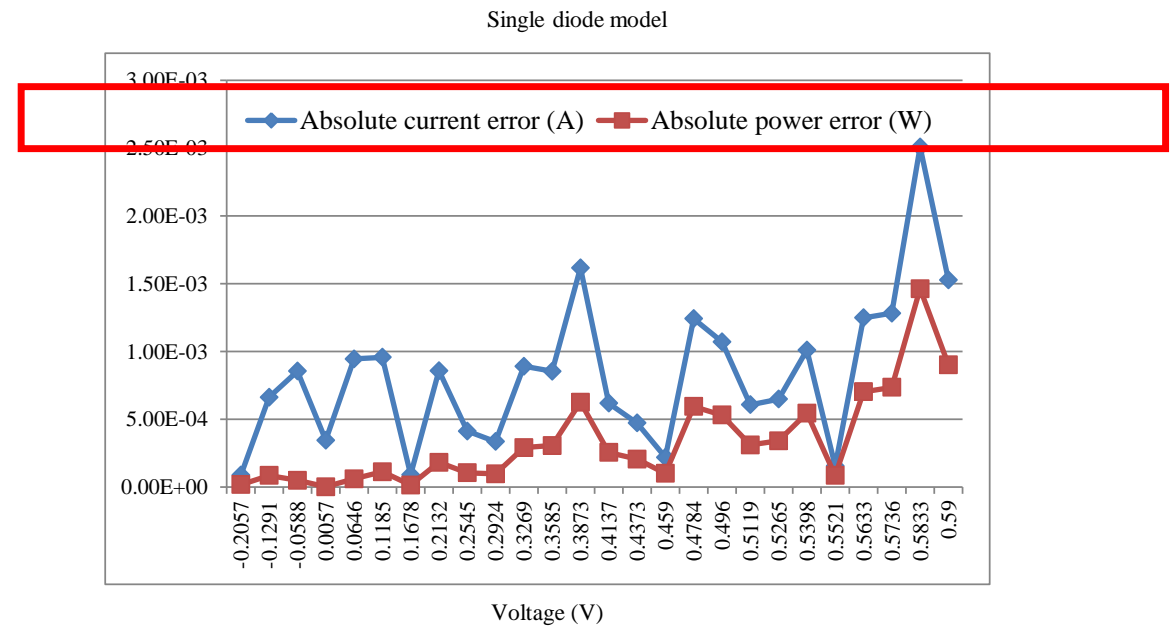

Fig. 7 Individual absolute errors for current and power using pSFS (single diode model) 
Double diode model

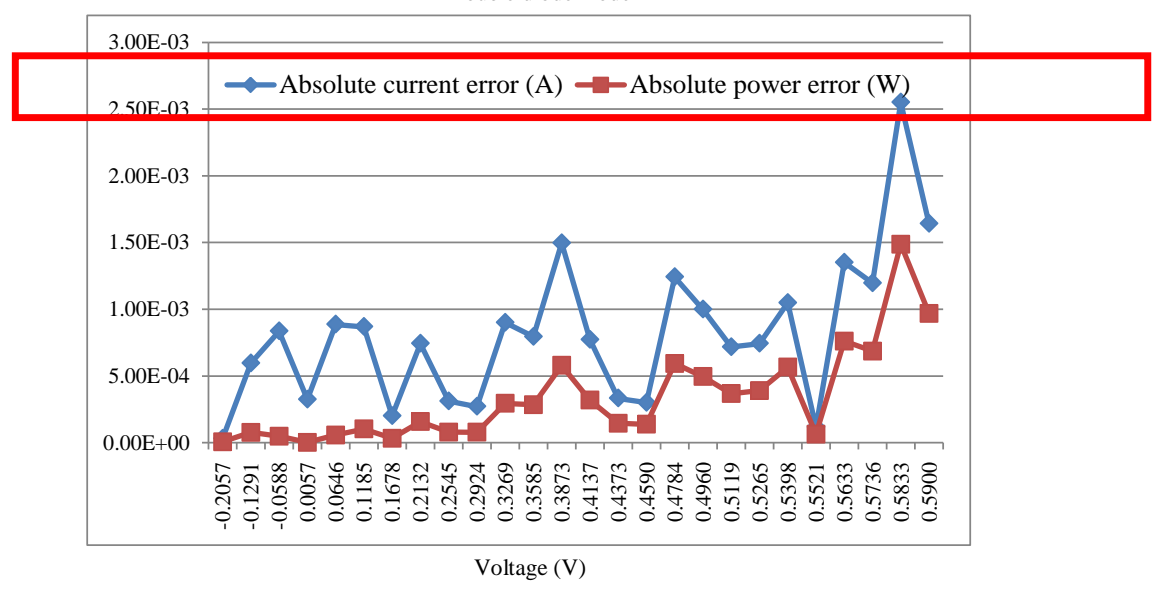

Fig. 9 Individual absolute errors for current and power using pSFS (double diode model)

PV module model

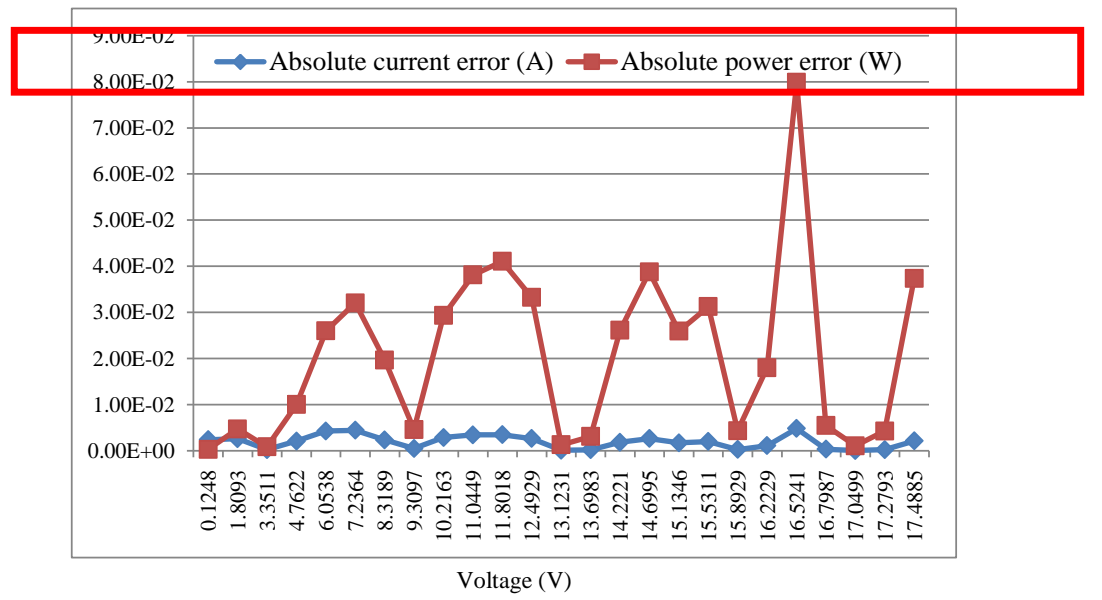

Fig. 11 Individual absolute errors for current and power using pSFS (PV module model)

7. Equation fonts are different from text font. Also, the font size is not similar in the text. Make the font type and size consistent along the whole manuscript.

Our response: Thanks for your useful comments. We have carefully checked all the equations. Eqs(1),(2),(4),(5),(9) (16) have the normal font size, while Eqs. (3), (6) (8) have small font size. This is because Eqs. (3), (6) (8) have large size, the compiling software latex reduces the font size of these equations. These have been fixed. The font size in tables (1) (11) is meant to be smaller than other texts, following most journal styles.

8. In the introduction section there are parts that need to edit grammar and vocabulary Our response: Thank you for the comment. We have improved the writing. 


\section{Response to Reviewer \#2:}

The introduction of the paper is too long and should be broken down into introduction and literature review sections.

Our response: Thank you for your suggestions. To make a smooth reading, we have restructured the introduction, presenting it as the literature review, the contribution, and the organization. The meta-heuristic algorithms (MHAs) in literature review are divided into three separate groups: the classic, the latest, and the hybrid MHAs.

\section{Response to Reviewer \#3:}

The authors presented a novel algorithm employing the use of stochastic fractal search with chaotic elitist perturbation. The proposed model shows some potential with a simple and yet efficient approach in the diffusion and search process. There are, however, some concerns on the current paper:

1. The manuscript must be sent to a proofreader. Some grammatical and stylistic errors were found, i.e. "Till now," page 2 line 4 and "...parameters march well.." page 23, line 50.

Our response: Thank you for reading our paper carefully. We've checked and improved the writing all through the paper.

2. Add a nomenclature table.

Our response: Thanks for your comments. Following your suggestion, we have added a nomenclature and abbreviation table in the revised manuscript. Please find the added table in our responses to Reviewer \#1.

3. In section 1, the different categories of the MHAs are presented. But, they lack a meaningful context. The authors should try to discuss the various types of MHAs with in-depth summaries, discuss the advantage/disadvantage of the MHAs, the basic methodology/concept behind them. This is to have a proper flow from one paragraph to another.

Our response: Thank you for the insightful comments. In the revised manuscript, we have thoroughly re-written the literature review, and added in-depth views MHAs following a proper logic. Please see "Section 1.1 Literature review" for details. 
4. The results shown are compared against other developed algorithms and show some potentials when compared against them. However, in section 4.1 to 4.3 , because the RMSE values are very close between the pSFS, SFS and TLABC, I would suggest the authors should include further comparison data between the experimental values and the other algorithm (SFS and TLABC) to strengthen the claim (as evidence). Plus, discuss why the need for pSFS when the other algorithm show similar results?

Our response: Thank you for the careful reading. In the original manuscript, Sections 4.1 to 4.3, the comparisons are made based on the best RMSE values among 30 independent runs for three diode models. The experimental values from pSFS, SFS and TLABC are close to each other, but these data cannot be used to assess the average accuracy and robustness of the algorithms. The statistical results are used instead.

In the revised manuscript, we restructure the section of "Results and analysis". The original sections 4.1 to 4.3 are merged into one single section, Section 4.1, "Comparisons based on the best results". Section 4.2 gives the "Comparisons based on the statistical results".

From the statistical results in Section 4.2, it can be concluded that the proposed pSFS achieves the overall best parameter estimation accuracy and robustness among the eight algorithms.

5. In section 4.6.1 and 4.6.2, please further explain why the different population size yields different results for the currently proposed method (instead of just reporting the data). The discussion must be critical with a strong mathematical/engineering logic.

Our response: That makes good sense. Thanks. In the revised manuscript, we have explained the impacts of parameters in the proposed method to the estimation results. See Section 4.4 in for details. 


\section{*Highlights}

\section{Highlight}

- A new algorithm pSFS is proposed for solar photovoltaic parameter estimation

- The pSFS employs the diffusion and updating based search operators

- Chaotic elitist perturbation strategy is used to enhance the searching efficiency

- The pSFS outperforms the recently-developed algorithms in terms of accuracy and robustness 


\title{
Perturbed stochastic fractal search for solar PV parameter estimation
}

\begin{abstract}
Following the widespread use of solar energy all over the world, the design of high quality photovoltaic (PV) cells has attracted strong research interests. To properly evaluate, control and optimize solar PV systems, it is crucial to establish a reliable and accurate model, which is a challenging task due to the presence of non-linearity and multi-modality in the PV systems. In this work, a new meta-heuristic algorithm (MHA), called perturbed stochastic fractal search (pSFS), is proposed to estimate the PV parameters in an optimization framework. The novelty lies in two aspects: (i) employ its own searching operators, i.e., diffusion and updating, to achieve a balance between the global exploration and the local exploitation; and (ii) incorporate a chaotic elitist perturbation strategy to improve the searching performance. To examine the effectiveness of pSFS, this method is applied to solve three PV estimation problems for different PV models, including single diode, double diode and PV modules. Experimental results and statistical analysis show that the proposed pSFS has improved estimation accuracy and robustness compared with several other algorithms recently developed.
\end{abstract}

Keywords: Photovoltaic (PV) modeling, parameters estimation, stochastic fractal search, chaotic elitist perturbation

\section{Introduction}

Solar energy is considered to be a promising renewable energy because of its affluent availability and cleanliness. Solar photovoltaic (PV) systems can convert solar energy into electricity and supply power. It has been widely used for several decades and the rapid growth is still continuing [1].

Mathematical models are important for control, optimization and assessment of solar PV systems [2]. Several PV models have been developed, among which the single diode model (SDM) and the double diode model (DDM) are most widely used [3. The prediction quality of a PV model largely depends on the extracted model parameters. Therefore, accurate and robust estimation of model parameters is crucial for PV modeling.

\subsection{Literature review}

PV parameter estimation is often formulated as an optimization problem to minimize the residual errors statistically. Due to the non-linearity inherent in the dynamics and the noise involved in the experimental

\footnotetext{
*Corresponding author

Email addresses: xuchen@ujs.edu.cn (Xu Chen), hong.yue@strath.ac.uk (Hong Yue), yukunjie@zzu.edu.cn (Kunjie Yu)
} 


\begin{tabular}{lll} 
Diode ideality constant & $k$ & Boltzmann constant $\left(1.3806503 \times 10^{23} J / K\right)$ \\
Problem dimension & $N P$ & Population size \\
Function evaluations & $P_{b e s t}$ & Position of the best particle \\
Maximum number of function evaluations & $P_{i}$ & Position of the $i$-th particle \\
Maximum number of generations & $q$ & Electron charge $\left(1.60217646 \times 10^{-19} C\right)$ \\
Diode current $(\mu \mathrm{A})$ & $R_{S}$ & Series resistance $(\Omega)$ \\
Output current (A) & $R_{s h}$ & Shunt resistance $(\Omega)$ \\
Photo-generated current (A) & $T$ & Cell temperature (K) \\
Reverse saturation current (A) & $V_{L}$ & Cell output voltage (V) \\
Diffusion current (A) & $V_{t}$ & Junction thermal voltage (V) \\
Saturation current (A) & $\mu_{B P}, \mu_{P}$ & Gaussian parameters \\
shunt resistor current (A) & $\gamma_{i}$ & Selection probability for the $i$-th particle \\
& & \\
Artificial bee colony & MDN & Maximum diffusion number \\
Biogeography-based heterogeneous cuckoo search & MHA & Meta-heuristic algorithms \\
Biogeography-based learning PSO & pSFS & Perturbed stochastic fractal search \\
Comprehensive learning PSO & P-DE & Penalty based differential evolution \\
Double diode mode & PSO & Particle swarm optimization \\
Differential evolution & PV & Photovoltaic \\
Enhanced leader PSO & RMSE & Root mean square error \\
Flower pollination algorithm & SD & Standard deviation \\
Fireworks algorithm & SDM & Single diode model \\
Generalized opposition TLBO & SFS & Stochastic fractal search \\
Hybrid firefly algorithm and pattern search & SSA & Salp swarm algorithm \\
Harmony search & TLBO & Teaching-learning-based optimization \\
Individual absolute current error & TLABC & Teaching-learning-based artificial bee colony \\
Improved adaptive differential evolution & TRR & Trust-region reflective \\
Individual absolute power error & WOA & Whale optimization algorithm \\
\hline & &
\end{tabular}

current-voltage $(I-V)$ data, PV parameter estimation is usually a multimodal problem with multiple local optimums [4. Deterministic techniques have been proposed for solving this problem, including the Lambert W-functions [5], the Newton-Raphson method [6], and the iterative curve fitting [7. Most of the deterministic techniques are based on the gradient information. They show powerful local search abilities, but likely fall into local optimums. In addition, deterministic techniques require strict conditions such as the differentiability and convexity, which restricts their wide applications.

More recently, meta-heuristic algorithms (MHAs) have been developed and employed for PV parameters estimation problems. Inspired by natural phenomena, MHAs have advantages in solving complex global optimization problems [8, 9, and have no requirements such as continuity, differentiability or convexity for the optimization problems [10, 11. There are three groups of MHAs used for parameter estimation, i.e., the classic MHAs, the latest MHAs and the hybrid MHAs.

The classic MHAs include algorithms such as differential evolution (DE), particle swarm optimization (PSO), harmony search (HS), artificial bee colony (ABC) and teaching-learning-based optimization (TLBO). DE has a simple structure and exhibits good accuracy, but its optimization results highly depend on two control parameters, namely the scaling factor and the crossover rate. In [12, the fitness information was used to tune the control parameters, and an improved adaptive DE (IADE) was proposed for estimating parameters of PV models. PSO is also simple to implement and has fast convergence speed, but it often suffers from premature convergence. To overcome this drawback, several PSO variants including the enhanced leader PSO (ELPSO) [13], the adaptive mutation strategy (MPSO) [14] and the chaotic heterogeneous comprehensive learning PSO (CHCLPSO) [15, have been developed and applied to extract the PV model parameters. In [16], three HS variants were used to determine the unknown parameters of solar cell models. The simulation studies show that HS algorithms achieve improved results compared to the simulated annealing and the pattern search. In [17, artificial bee colony $(\mathrm{ABC})$ algorithm was used to identify the parameters of two solar cells. It is observed that 
ABC has good search ability for multimodal objective functions compared with HS, PSO, genetic algorithm and bacterial foraging algorithm. In [18, the generalized oppositional TLBO (GOTLBO) algorithm was proposed to identify the parameters of solar cell models. By using the generalized opposition-based learning, the GOTLBO algorithm accelerates the convergence speed compared to the basic TLBO.

There is not a single classic MHA that is competent for all optimization problems. The latest MHAs are used for PV parameter estimation. In [19, the whale optimization algorithm (WOA) was proposed for PV parameters estimation, in which the chaotic map was used to automatically adjust the internal parameters of WOA. This helps to avoid local optimum and also improves the convergence rate. In 20, two prey searching strategies were embedded into WOA, which overcomes the problem of premature convergence, and the improved WOA was applied to estimate the model parameters of two practical PV power stations. Alam et al. 3] proposed a flower pollination algorithm (FPA) based method for PV parameters estimation method to improve the convergence performance. Improved JAYA algorithms were proposed to accurately and reliably identify the parameters of several PV models [4, 21. However, JAYA algorithms need relatively large number of iterations to converge. Several other latest MHAs including the salp swarm algorithm (SSA) 22] and the fireworks algorithm (FWA) 23. were also utilized to extract PV model parameters, and achieve competitive performance in the reported case studies.

Hybrid MHAs combine advantages from more than one MHA, thus provide more accurate parameters than the individual algorithms. Several hybrid MHAs were also developed for PV parameter estimation problems, including the hybrid firefly algorithm and pattern search (HFAPS) [24, the teaching-learning-based artificial bee colony (TLABC) 25, the biogeography-based heterogeneous cuckoo search (BHCS) 26, and the trustregion reflective artificial bee colony (ABC-TRR) [27.

\subsection{Contribution}

The above short literature review shows that parameter estimation of PV models is still a challenging task that requires more effective and efficient tools. Recent development suggests that MHAs have good potential for parameter estimation of PV models. In this work, we will explore a particular MHA, the stochastic fractal search (SFS), for PV modeling.

SFS is an MHA which uses the diffusion and update processes based on random fractal properties [28. This algorithm is developed to overcome the weaknesses of MHAs such as premature convergence and low robustness. SFS has been applied in solving several real-world problems, such as system reliability optimization [29, PID controller design for an automatic voltage regulator system [30, and electric power economic dispatch [31].

Inspired by the recent progress of SFS in applications, in this study, we investigate the further development of SFS for the challenging PV parameter estimation problems. Specifically, we propose a perturbed stochastic fractal search (pSFS) algorithm, which employs the diffusion and updating operators in searching. Moreover, a simple but efficient chaotic elitist perturbation is incorporated into the pSFS, which drives the local search around the best solution during the searching process. This designed perturbation can further improve the solution accuracy and robustness. The proposed pSFS is applied to solve three PV parameter estimation problems with different diode models, and the results are compared with several recently-developed MHAs to 
verify its performance.

The contributions of this paper are listed as follows:

(1) A new perturbed SFS (pSFS) approach is proposed for solving PV parameter estimation problems.

(2) A chaotic elitist perturbation strategy is implemented to improve the search efficiency.

(3) pSFS is applied to three PV parameter estimation problems with different diode models, as well as three PV modules using survey data.

(4) By comparing with the recently-developed algorithms, the superiority of pSFS in solution accuracy and robustness are demonstrated.

\subsection{Paper organization}

The remaining of this paper is organized as follows. In Section 2, three PV parameter estimation problems are defined for SDM, DDM and PV module, respectively. The basic SFS and the proposed pSFS algorithms are presented in Section 3. Comparisons are made in Section 4 between the pSFS and several recently-developed algorithms. In Section 5, the practical use of pSFS is examined by using surveyed experimental data from three different PV modules. Finally, conclusions are given in Section 6.

\section{Problem statement}

This section presents the mathematical formulation of PV parameter estimation problems with three different diode models, i.e., SDM, DDM, and PV module models.

\subsection{Single diode model}

The equivalent electric circuit of a SDM is shown in Fig.1. In SDM, the output current $I_{L}$ is calculated as follows [32, 33]:

$$
I_{L}=I_{p h}-I_{d}-I_{s h}=I_{p h}-I_{s d} \cdot\left[\exp \left(\frac{V_{L}+R_{S} \cdot I_{L}}{a \cdot V_{t}}\right)-1\right]-\frac{V_{L}+R_{S} \cdot I_{L}}{R_{s h}}
$$

where $I_{p h}, I_{d}$ and $I_{s h}$ are the photo-generated current, diode current and shunt resistor current, respectively; $V_{L}$ is the cell output voltage; $I_{s d}$ is the reverse saturation current; $R_{S}$ and $R_{s h}$ are the series resistance and shunt resistance, respectively; $a$ is the diode ideality constant; and $V_{t}$ is the junction thermal voltage calculated as follows:

$$
V_{t}=\frac{k T}{q}
$$

where $k=1.3806503 \times 10^{23} \mathrm{~J} / \mathrm{K}$ is the Boltzmann constant, $q=1.60217646 \times 10^{-19} \mathrm{C}$ is the electron charge, and $T$ is the cell temperature $(K)$. 


\subsection{Double diode model}

The equivalent electric circuit of the DDM is shown in Fig.2. In DDM, the cell output current $I_{L}$ can be calculated as follows 32,33 :

$$
\begin{aligned}
I_{L} & =I_{p h}-I_{d 1}-I_{d 2}-I_{s h} \\
& =I_{p h}-I_{s d 1} \cdot\left(\exp \left(\frac{V_{L}+I_{L} R_{S}}{a_{1} V_{t}}\right)-1\right)-I_{s d 2} \cdot\left(\exp \left(\frac{V_{L}+I_{L} R_{S}}{a_{2} V_{t}}\right)-1\right)-\frac{V_{L}+I_{L} R_{s}}{R_{s h}}
\end{aligned}
$$

where $I_{d 1}$ and $I_{d 2}$ denote the first and the second diode currents, respectively; $I_{s d 1}$ and $I_{s d 2}$ denote the diffusion and saturation currents, respectively; $a_{1}$ and $a_{2}$ are the diffusion and recombination diode ideality factors, respectively.

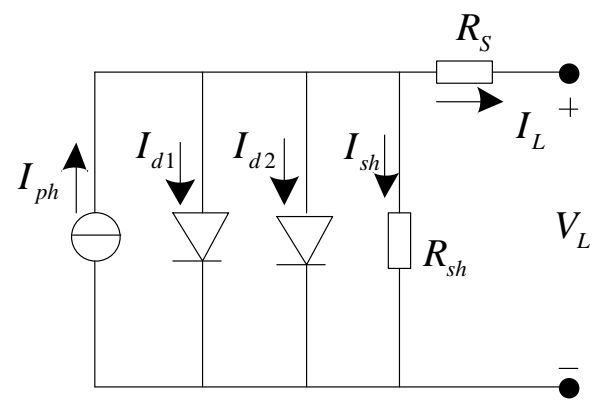

Figure 2: Equivalent electric circuit of double diode model

\subsection{PV module model}

The PV module model consists of $N_{s} \times N_{p}$ solar cells connected in series and/or in parallel as shown in Fig.3. The cell output current $I_{L}$ can be formulated as follows [32, 33]:

$$
I_{L}=N_{p} \cdot I_{p h}-N_{p} \cdot I_{s d} \cdot\left[\exp \left(\frac{V_{L} / N_{S}+R_{S} \cdot I_{L} / N_{p}}{a \cdot V_{t}}\right)-1\right]-\frac{N_{p} \cdot V_{L} / N_{S}+R_{S} \cdot I_{L}}{R_{s h}}
$$

where $N_{p}$ and $N_{s}$ are the numbers of solar cells in parallel and in series, respectively. 


\subsection{Objective function of $P V$ estimation problem}

In order to extract the unknown parameters of PV models based on the experimental data, the PV parameter estimation is converted into an optimization problem. The objective function is defined as the overall root mean square error (RMSE) between experimental data and simulated data as follows [18, 34]:

$$
\operatorname{RMSE}(\boldsymbol{x})=\sqrt{\frac{1}{N} \sum_{k=1}^{N} f_{k}\left(V_{L}, I_{L}, \boldsymbol{x}\right)^{2}}
$$

where $N$ is the number of experimental data, and $\boldsymbol{x}$ is the vector of unknown model parameters.

For the SDM,

$$
\begin{gathered}
f_{k}\left(V_{L}, I_{L}, \boldsymbol{x}\right)=I_{p h}-I_{s d}\left(\exp \left(\frac{V_{L}+I_{L} R_{S}}{a V_{t}}\right)-1\right)-\frac{V_{L}+I_{L} R_{s}}{R_{s h}}-I_{L} \\
\boldsymbol{x}=\left\{I_{p h}, I_{s d}, R_{S}, R_{s h}, a\right\}
\end{gathered}
$$

For the DDM,

$$
\begin{gathered}
f_{k}\left(V_{L}, I_{L}, \boldsymbol{x}\right)=I_{p h}-I_{s d 1}\left(\exp \left(\frac{V_{L}+I_{L} R_{S}}{a_{1} V_{t}}\right)-1\right)-I_{s d 2}\left(\exp \left(\frac{V_{L}+I_{L} R_{S}}{a_{2} V_{t}}\right)-1\right)-\frac{V_{L}+I_{L} R_{s}}{R_{s h}}-I_{L} \\
\boldsymbol{x}=\left\{I_{p h}, I_{s d 1}, I_{s d 2}, R_{S}, R_{s h}, a_{1}, a_{2}\right\}
\end{gathered}
$$

For the PV module model,

$$
\begin{gathered}
f_{k}\left(V_{L}, I_{L}, \boldsymbol{x}\right)=N_{p} I_{p h}-N_{p} I_{s d}\left[\exp \left(\frac{V_{L} / N_{S}+R_{S} I_{L} / N_{p}}{a V_{t}}\right)-1\right]-\frac{N_{p} V_{L} / N_{S}+R_{S} I_{L}}{R_{s h}}-I_{L} \\
\boldsymbol{x}=\left\{I_{p h}, I_{s d}, R_{S}, R_{s h}, a\right\}
\end{gathered}
$$




\section{Perturbed stochastic fractal search for $\mathbf{P V}$ model estimation}

\subsection{Stochastic fractal search}

SFS is a recently developed MHA proposed by Salimi [28, taking inspiration from the natural growth phenomenon of random fractal. The SFS algorithm mainly uses two processes namely diffusion and updating to improve the searching. In the diffusion process, each particle (i.e., candidate solution) diffuses around its own location, and carries out the exploitation task. By contrast, in the updating process, each particle is updated according to the location of other particles, and this process leads to exploration properties. The two processes of SFS are shown in Fig. 4.
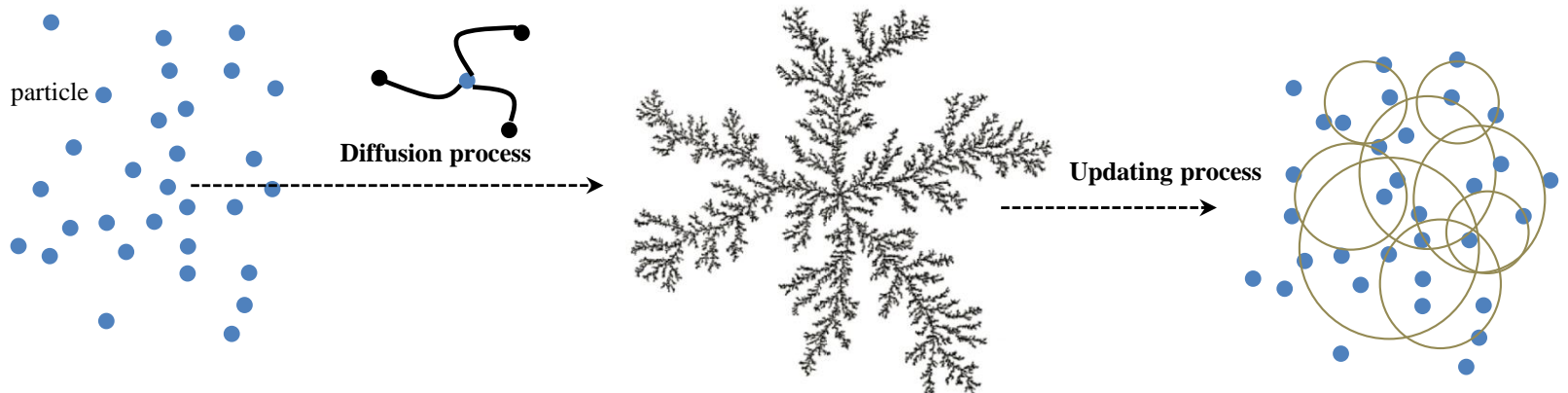

Figure 4: The main procedure of SFS algorithm

The diffusion process uses Gaussian random walks to generate points around each particle until a predetermined maximum diffusion number $(\mathrm{MDN})$ is reached. There are two types of Gaussian walks in the diffusion process, which are described as follows:

$$
\begin{gathered}
G W_{1}=\operatorname{Gaussian}\left(\mu_{B P}, \delta\right)+\left(\operatorname{rand}(0,1) \times P_{\text {best }}-\operatorname{rand}(0,1) \times P_{i}\right) \\
G W_{2}=\operatorname{Gaussian}\left(\mu_{P}, \delta\right)
\end{gathered}
$$

where $P_{i}$ and $P_{b e s t}$ are the positions of the $i$-th and the best particles, respectively; $i=1,2, \cdots, N P, N P$ is the population size; $\operatorname{rand}(0,1)$ is a random number generated within $[0,1]$. Gaussian parameters $\mu_{B P}$ and $\mu_{P}$ are equal to $P_{\text {best }}$ and $P_{i}$, respectively. The standard deviation $\delta$ is dynamically adjusted based on the number of the generation $G$ :

$$
\delta=\left|\frac{\log (G)}{G} \times\left(P_{i}-P_{\text {best }}\right)\right|
$$

The update process employs two statistical procedures to undertake the exploration in SFS. In the first updating process, each particle position is updated as follows:

$$
P_{i}^{\prime}(j)=\left\{\begin{array}{l}
P_{r 1}(j)-\operatorname{rand}(0,1) \times\left(P_{r 2}(j)-P_{i}(j)\right) \text { if } \gamma_{i}<\operatorname{rand}(0,1) \\
P_{i}(j) \quad \text { otherwise }
\end{array}\right.
$$

where $j \in\{1,2, \cdots, D\}$ is the index for each optimization variable; $D$ is the number of optimization variables; 
$P_{i}^{\prime}$ is the new position of $P_{i} ; P_{r 1}$ and $P_{r 2}$ are the positions of two randomly selected particles; $\gamma_{i}$ is the selection probability for particle $P_{i}$ that can be calculated as:

$$
\gamma_{i}=1-\frac{\operatorname{rank}\left(P_{i}\right)}{N P}
$$

where $\operatorname{rank}\left(P_{i}\right)$ is the fitness order from the best to the worst of the $i$-th particles in the population. Obviously, the worse particle has a smaller $\gamma_{i}$ undergoing the first update process.

In the second updating process, the probability $\gamma_{i}$ is recalculated and used to determine whether a particle $P_{i}$ should be updated as follows:

$$
P_{i}^{\prime}(j)= \begin{cases}P_{i}(j)-\operatorname{rand}(0,1) \times\left(P_{r 1}^{\prime}(j)-P_{\text {best }}(j)\right) & \text { if } \operatorname{rand}(0,1)<0.5 \\ P_{i}(j)+\operatorname{rand}(0,1) \times\left(P_{r 1}^{\prime}(j)-P_{r 2}^{\prime}(j)\right) & \text { otherwise }\end{cases}
$$

where $P_{r 1}^{\prime}$ and $P_{r 2}^{\prime}$ are the positions of two randomly selected particles.

\subsection{Perturbed stochastic fractal search}

\subsubsection{Chaotic elitist perturbation strategy}

During the search process of SFS, the best solution found is recorded in each generation. Its quality affects both the search process and the final solution. Chaotic elitist perturbation strategy is introduced to perform adaptive local search around the best solution as follows:

$$
P^{*}(j)=\left\{\begin{array}{l}
P_{\text {best }}(j)+\operatorname{rand}(0,1) \cdot\left(2 z_{k}-1\right) \quad \text { if } \operatorname{rand}(0,1)<1-F E S / F E S_{\max } \\
P_{\text {best }}(j) \text { otherwise }
\end{array}\right.
$$

where the logistic map $z_{k}=4 z_{k-1}\left(1-z_{k-1}\right)$ is used to generate the $k$-th chaotic iteration value, with its initial value $z_{0}$ randomly generated within $[0,1] ; F E S$ and $F E S_{\max }$ are the current and maximum number of function evaluations. The new solution $P^{*}$ is compared with the worst solution $P_{\text {worst }}$ in current population, and the better one is selected for the next generation.

According to Eq. (18), the chaotic elitist perturbation strategy can perform a self-adaptive perturbation during the optimization process. To be specific, the value of FES is relative small in the early search stage, thus more perturbations will be added to the best solution, which is useful for global exploration. By contrast, the value of $F E S$ is close to 1 in the latter search stage, therefore more information will be inherited from the best solution, which is beneficial for local exploitation.

\subsubsection{Algorithmic framework}

The flowchart of the proposed pSFS algorithm is presented in Fig.5. A more detailed description of pSFS using the pseudocode is presented in Appendix 1. It can be seen that, the proposed pSFS takes four searching stages, including the diffusion process, the first and second updating processes, and the chaotic elitist perturbation. These four stages have different roles in the searching process. The diffusion process focuses on 


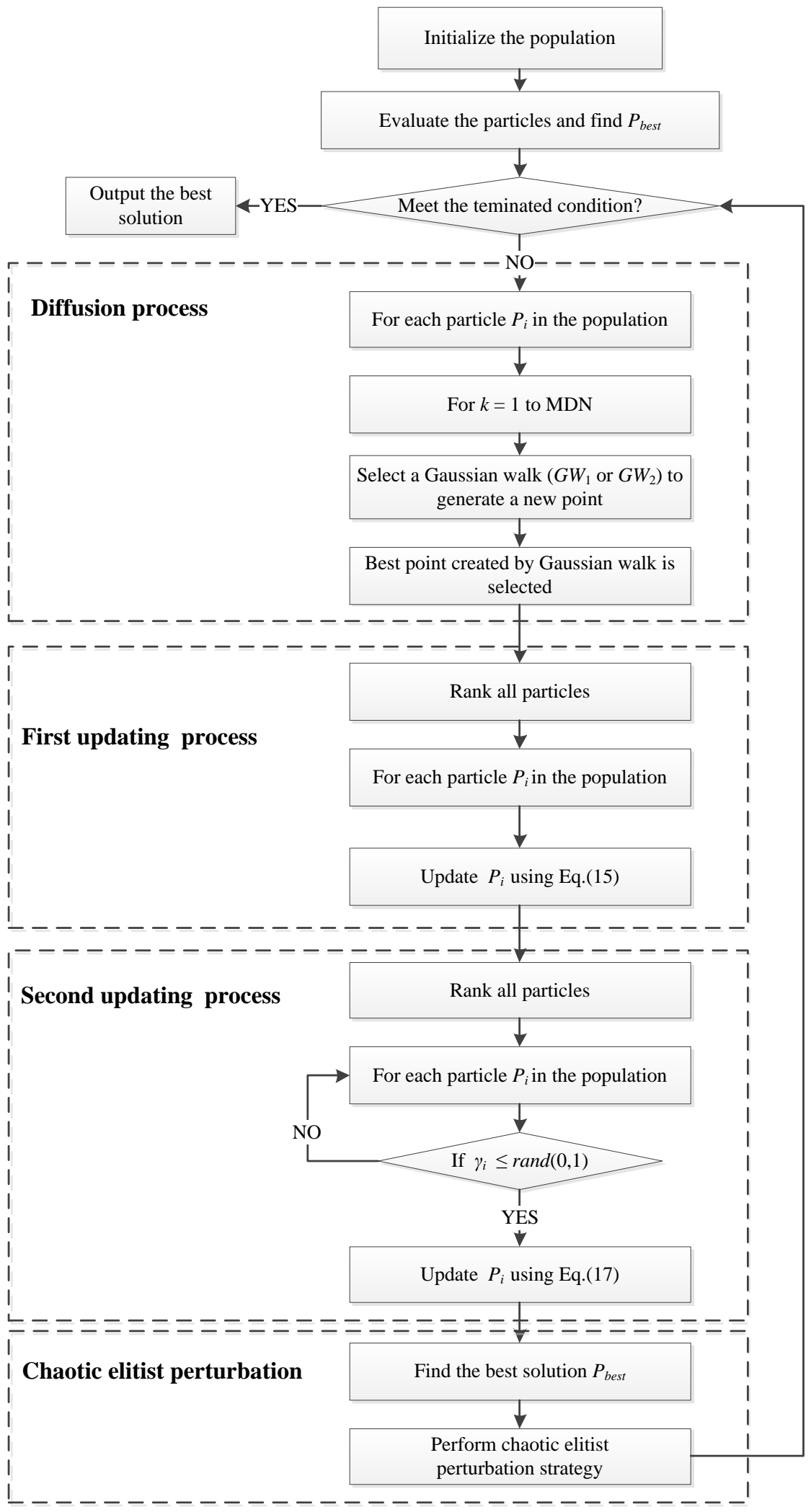

Figure 5: Flowchart of the pSFS algorithm 
local exploitation, the two updating processes focus on global exploration, and the chaotic elitist perturbation strategy performs adaptive local search to further improve the solution accuracy and robustness.

The computational complexity of pSFS mainly includes: (a) the time for the diffusion process $T_{d i}$, (b) the time for the first updating process $T_{u p 1}$, (c) the time for the second updating process $T_{u p 2}$, and (d) the time for the chaotic elitist perturbation strategy $T_{p e}$. The total computational complexity of pSFS can be represented by a time metric as follows:

$$
\begin{aligned}
T_{p S F S}= & \left(T_{d i}+T_{u p 1}+T_{u p 2}+T_{p e}\right) \cdot G_{\max } \\
= & (O(N P \cdot M D N \cdot D)+O(N P \cdot \log (N P)+N P \cdot D) \\
& +O(N P \cdot \log (N P)+N P \cdot D)+O(D)) \cdot G_{\max } \\
= & O(M D N \cdot D+2 \log (N P)+2 D) \cdot N P \cdot G_{\max } \\
= & O(M D N \cdot D+2 \log (N P)+2 D) \cdot F E S_{\max }
\end{aligned}
$$

where $D$ is the problem dimension, $N P$ is the population size, $M D N$ is the maximum diffusion number, $G_{\max }$ is the maximum number of generations, and $F E S_{\max }$ is the maximum number of function evaluations.

\section{Results and analysis}

The proposed pSFS algorithm is evaluated by solving three PV parameter estimation problems as described in Section 2. The experimental current-voltage data of the SDM and DDM are measured from a RTC France solar cell (under $1000 \mathrm{~W} / \mathrm{m}^{2}$ at $33{ }^{\circ} \mathrm{C}$ ) and are taken from [6]. The experimental current-voltage data of the PV module are from Photowatt-PWP201 module (under $1000 \mathrm{~W} / \mathrm{m}^{2}$ at $45{ }^{\circ} \mathrm{C}$ ) $[\underline{6}$. The searching ranges for the model parameters are listed in Table 1, which are the same as those in [4, 35, 36. This will ensure comparisons are made under the same system settings.

The pSFS is compared with seven recently-developed algorithms: basic SFS 28], CLPSO (comprehensive learning PSO) [37, BLPSO (biogeography-based learning PSO) [38, ABC (artificial bee colony) [39, GOTLBO (generalized oppositional TLBO) [18], TLABC (teaching-learning-based ABC) [25], and IJAYA (improved JAYA) 33. These algorithms show good performance for PV parameter estimation, therefore chosen for comparison in this study.

The parameter settings for these algorithms under comparison are given in Table 2, mainly taken from their corresponding literature. The tuning parameters for the proposed pSFS are also given in Table 2. The maximum number of function evaluations is set to be $F E S_{\max }=50000$ for all three PV parameter estimation problems 25, 4, 36. All the algorithms are coded in MATLAB and run 30 times independently to obtain the statistical results. The experiments are carried out on a DELL computer with a Core i5-4460 processor and 8G RAM.

\subsection{Comparisons based on the best results \\ 4.1.1. Results on the single diode mode}

We first compare the proposed pSFS with the other seven algorithms on the SDM. There are five unknown parameters to be estimated in the SDM model. Table 3 presents the comparison results for all these algorithms. 


\begin{tabular}{ccccc}
\multicolumn{2}{c}{ Table 1: Parameter boundaries of three PV models. } \\
\hline Parameter & \multicolumn{2}{c}{ SDM/DDM } & \multicolumn{2}{c}{ PV module } \\
& Lower & Upper & Lower & Upper \\
\hline$I_{p h}(\mathrm{~A})$ & 0 & 1 & 0 & 2 \\
$I_{s d}, I_{s d 1}, I_{s d 2}(\mu \mathrm{A})$ & 0 & 1 & 0 & 50 \\
$R_{S}(\Omega)$ & 0 & 0.5 & 0 & 2 \\
$R_{s h}(\Omega)$ & 0 & 100 & 0 & 2000 \\
$a, a_{1}, a_{2}$ & 1 & 2 & 1 & 50 \\
\hline
\end{tabular}

Table 2: Parameter settings for pSFS and the other algorithms.

\begin{tabular}{lll}
\hline Algorithm & Year & Parameter settings \\
\hline CLPSO & 2006 & $N P=40, w=0.9 \sim 0.2, c=1.496, m=5$ \\
BLPSO & 2017 & $N P=40, w=0.9 \sim 0.2, c=1.496, I=E=1$ \\
ABC & 2007 & $N P=50$, limit $=200$ \\
GOTLBO & 2016 & $N P=50$, Jr $=0.3$ \\
TLABC & 2018 & $N P=50$, limit $=200, F=\operatorname{rand}(0,1)$ \\
IJAYA & 2017 & $N P=20$ \\
SFS & 2015 & $N P=30, M D N=1$, Gaussian walk $G W_{1}$ \\
pSFS & proposed & $N P=30, M D N=1$, Gaussian walk $G W_{1}$ \\
\hline
\end{tabular}

The extracted model parameters are also presented. Note that, the results reported in Table 3 are the best RMSE values among 30 independent runs. The statistical results will be compared in Section 4.2.

From the results in Table 3, the proposed pSFS, together with SFS and TLABC achieve the best RMSE value (i.e., 9.8602E-4). The RMSE reflects the estimation accuracy of the algorithms. Thus, the highest estimation accuracy has been achieved by pSFS, SFS and TLABC. IJAVA achieves the RMSE value of 9.8603E04, followed by GOTLBO (9.8658E-04) and ABC (9.8815E-04). The performance of CLPSO and BLPSO stays on the lower end of the comparison, and their RMSE values are 9.9207E-04 and 1.1239E-03, respectively.

The extracted parameters of pSFS are used to plot the $I-V$ and $P-V$ curves. As shown in Fig.6, both $I-V$ and $P-V$ characteristics curves demonstrate that the estimated model curves are highly consistent with the experimental data. Besides, Fig.7 plots the individual absolute current error (IACE) and individual absolute power error (IAPE) over the whole voltage range. The maximal IACE value is smaller than 2.5E-3, and the maximal IAPE value is smaller than 1.5E-3. All these observations demonstrate the high estimation accuracy of the pSFS algorithm.

Table 3: Results of pSFS and the compared algorithms on the single diode model

\begin{tabular}{cccccccc}
\hline Algorithm & $I_{p h}(\mathrm{~A})$ & $I_{s d}(\mu \mathrm{A})$ & $R_{s h}(\Omega)$ & $R_{S}(\Omega)$ & $a$ & RMSE & Rank \\
\hline CLPSO & 0.76064 & 0.33454 & 56.03420 & 0.03623 & 1.48469 & $9.9207 \mathrm{E}-04$ & 7 \\
BLPSO & 0.76063 & 0.42518 & 62.58528 & 0.03523 & 1.50940 & $1.1239 \mathrm{E}-03$ & 8 \\
ABC & 0.76085 & 0.33016 & 53.59884 & 0.03629 & 1.48339 & $9.8815 \mathrm{E}-04$ & 6 \\
GOTLBO & 0.76077 & 0.32256 & 53.33877 & 0.03637 & 1.48106 & $9.8658 \mathrm{E}-04$ & 5 \\
TLABC & 0.76078 & 0.32302 & 53.71636 & 0.03638 & 1.48118 & $\mathbf{9 . 8 6 0 2 E - 0 4}$ & 1 \\
IJAYA & 0.76078 & 0.32304 & 53.71441 & 0.03638 & 1.48119 & $9.8603 \mathrm{E}-04$ & 4 \\
SFS & 0.76078 & 0.32302 & 53.71852 & 0.03638 & 1.48118 & $\mathbf{9 . 8 6 0 2 E - 0 4}$ & 1 \\
pSFS & 0.76078 & 0.32302 & 53.71852 & 0.03638 & 1.48118 & $\mathbf{9 . 8 6 0 2 E - 0 4}$ & 1 \\
\hline
\end{tabular}

The best results are highlighted in bold font. 


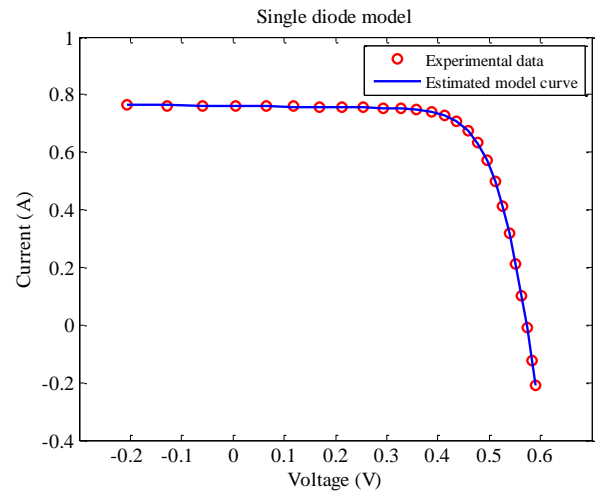

(a)

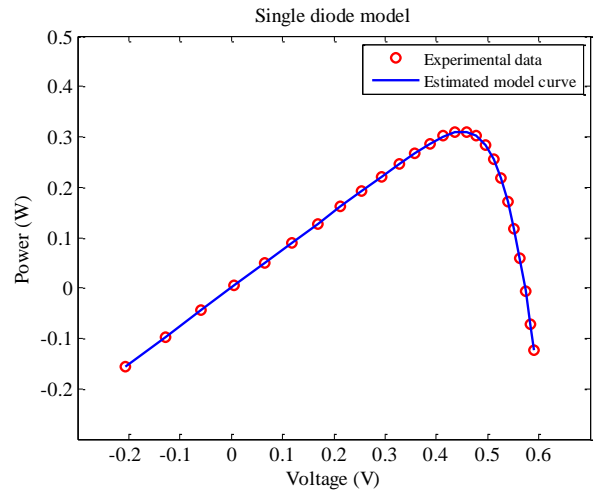

(b)

Figure 6: Comparisons between experimental data and the data estimated from pSFS for the single diode model: (a) $I$ - $V$ characteristics, (b) $P-V$ characteristics

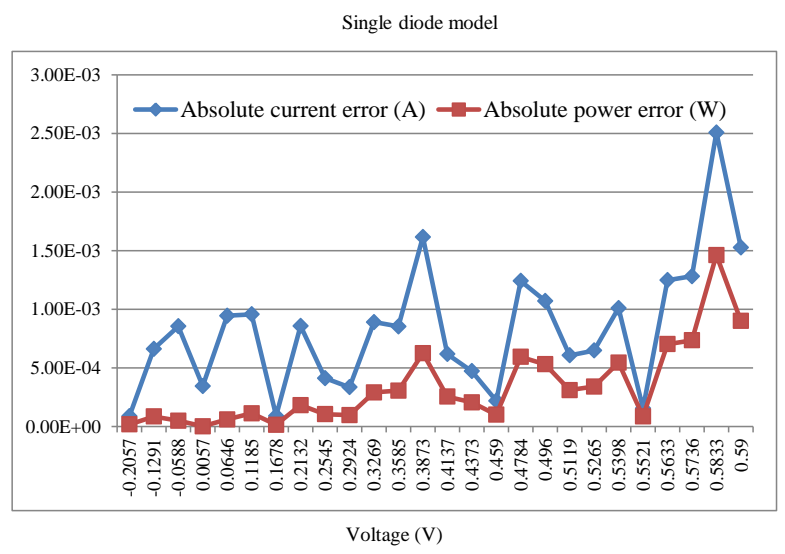

Figure 7: Individual absolute errors for current and power using pSFS (single diode model)

\subsubsection{Results on the double diode mode}

The comparison results of pSFS with the other seven algorithms for the DDM are presented in Table 4. Since there are seven unknown parameters for a DDM model, the estimation of the DDM is more complicated than that of the SDM.

From Table 4, it can be found that the proposed pSFS and the basic SFS attain the best RMSE value (i.e., 9.8255E-04). This indcates the higher accuracy of SFS algorithms over those non-SFS algorithms for PV parameter estimation. TLABC achieves the third best RMSE value (i.e., 9.8414E-04), and IJAVA obtains the fourth best RMSE value (i.e., 9.8423E-04). CLPSO and BLPSO exihit relative poor performance for this case, and their RMSE values are the worst among the eight algorithms.

To further evaluate the pSFS's estimation accuracy over the whole voltage range, Fig.8 plots the $I$ - $V$ and $P-V$ characteristic curves. The estimated model curves of pSFS are in good agreement with the experimental data. Meanwhile, from Fig.9, the maximal IACE value is less than 2.6E-3, and the maximal IAPE value is less than 1.5E-3. These results show that the proposed pSFS also achieves a very high estimation accuracy for the DDM. 


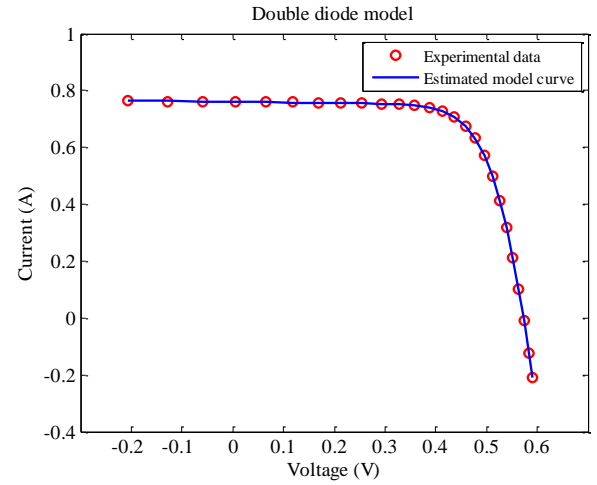

(a)

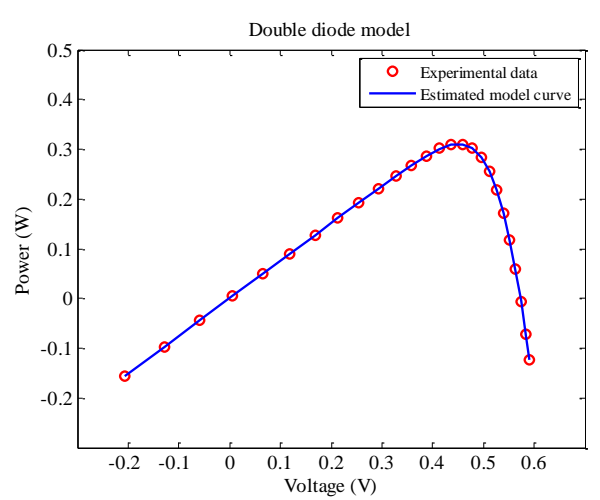

(b)

Figure 8: Comparisons between experimental data and the data estimated from pSFS for the double diode model: (a) $I$ - $V$ characteristics, (b) $P-V$ characteristics

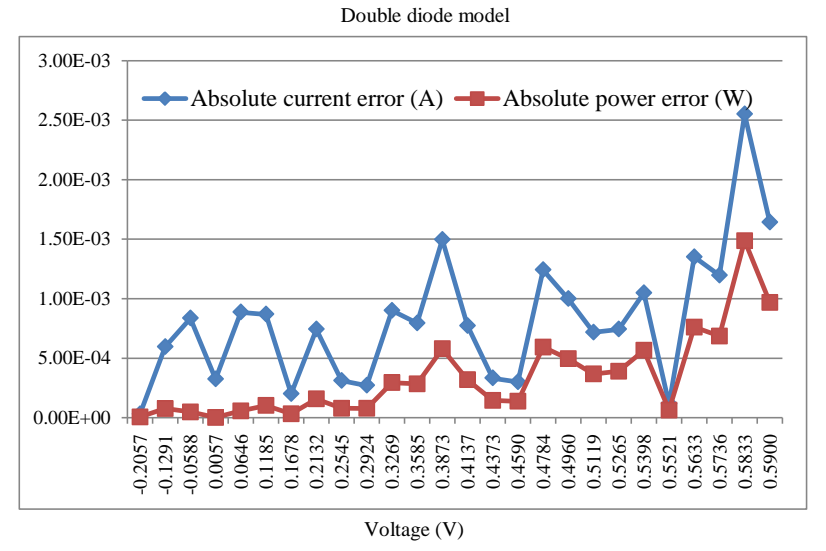

Figure 9: Individual absolute errors for current and power using pSFS (double diode model)

\subsubsection{Results on the PV module mode}

A PV module model that has five estimation parameters is taken to further evaluate the estimation accuracy of the proposed pSFS. Table 5 presents the comparison results of pSFS and the other algorithms. From the results in Table 5, three algorithms (i.e., pSFS, SFS and TLABC) achieve the best RMSE value (i.e., 2.42507E03), followed by IJAVA(2.42512E-03), GOTLBO(2.42513E-03), BLPSO (2.42520E-03), CLPSO (2.42661E-03) and $\mathrm{ABC}(2.44692 \mathrm{E}-03)$.

The optimal model parameters extracted by pSFS are used to construct the $I-V$ and $P-V$ curves. As 
shown in Fig 10, high consistency can be observed between the estimated model curves and the experimental data for both $I-V$ and $P-V$ characteristics. From Fig.11, it can be observed that the maximal IACE value is less than 8.0E-2, and the maximal IAPE value is less than 5.0E-3. All these comparisons demonstrate the high estimation accuracy of the proposed pSFS for the PV module model.

Table 5: Results of pSFS and the compared algorithms on the PV module model

\begin{tabular}{|c|c|c|c|c|c|c|c|}
\hline Algorithm & $I_{p h}(\mathrm{~A})$ & $I_{s d}(\mu \mathrm{A})$ & $R_{s h}(\Omega)$ & $R_{S}(\Omega)$ & $a$ & RMSE & Rank \\
\hline CLPSO & 1.03036 & 3.45208 & 1001.18353 & 1.20269 & 48.60837 & $2.42661 \mathrm{E}-03$ & 7 \\
\hline BLPSO & 1.03052 & 3.50258 & 983.15030 & 1.20062 & 48.66526 & $2.42520 \mathrm{E}-03$ & 6 \\
\hline $\mathrm{ABC}$ & 1.03008 & 3.30190 & 968.65121 & 1.20631 & 48.44085 & $2.44692 \mathrm{E}-03$ & 8 \\
\hline GOTLBO & 1.03046 & 3.49907 & 989.68885 & 1.20080 & 48.66113 & $2.42513 \mathrm{E}-03$ & 5 \\
\hline TLABC & 1.03052 & 3.48226 & 981.84265 & 1.20127 & 48.64284 & $2.42507 \mathrm{E}-03$ & 1 \\
\hline IJAYA & 1.03054 & 3.47922 & 981.13257 & 1.20145 & 48.63942 & $2.42512 \mathrm{E}-03$ & 4 \\
\hline SFS & 1.03051 & 3.48226 & 981.98237 & 1.20127 & 48.64283 & $2.42507 \mathrm{E}-03$ & 1 \\
\hline pSFS & 1.03051 & 3.48226 & 981.98223 & 1.20127 & 48.64283 & $2.42507 \mathrm{E}-03$ & 1 \\
\hline
\end{tabular}

The best results are highlighted in bold font.

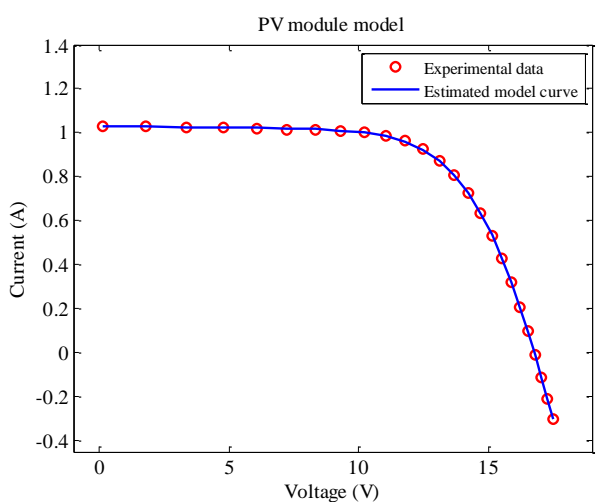

(a)

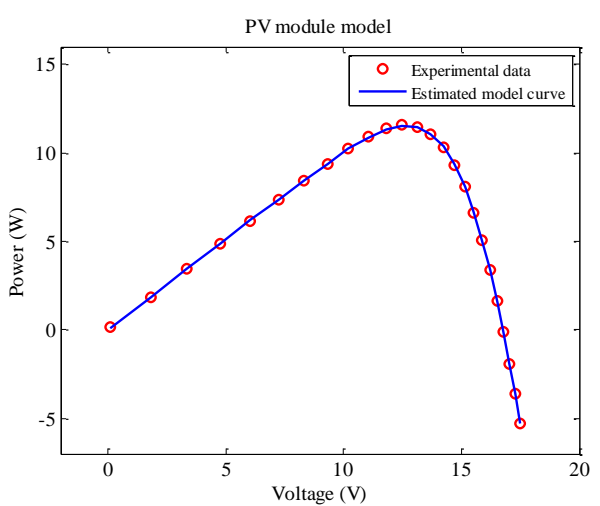

(b)

Figure 10: Comparisons between experimental data and the data estimated from pSFS for the PV module model: (a) $I$ - $V$ characteristics, (b) $P-V$ characteristics

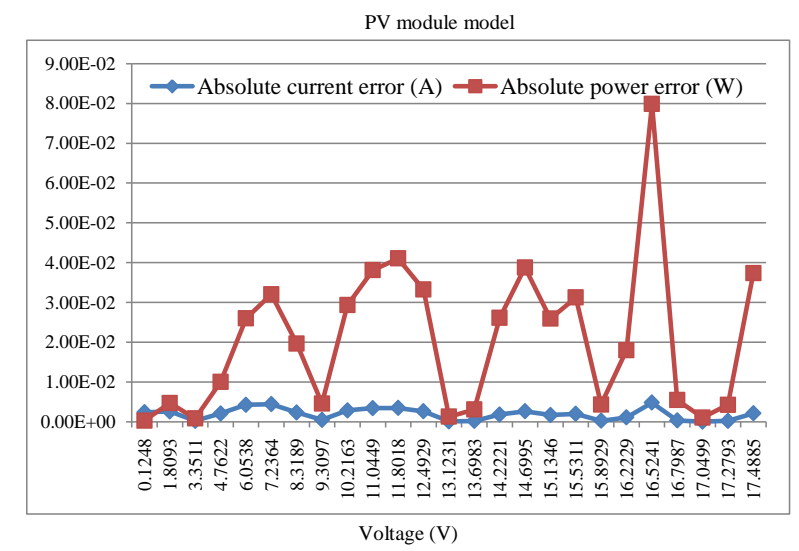

Figure 11: Individual absolute errors for current and power using pSFS (PV module model)

\subsection{Comparisons based on the statistical results}

Since all the eight PV parameter estimation algorithms are stochastic approaches, it is helpful to compare their statistical results. Table 6 presents the best, the mean, the worst and the standard deviation (SD) 
of RMSE of the eight algorithms over 30 independent runs. Again three PV models are considered in the comparison. In addition, the Wilcoxon rank sum test is conducted to compare pSFS with the other algorithms, so as to establish a statistical view. The symbols "+" and "=" indicate that pSFS performs significantly better or similar to others, with a significance level of $p=0.05$. The average computational times are also given in Table 6.

From Table 6, it can be found that:

- In terms of the best RMSE value, two SFS algorithms, i.e., pSFS and SFS, achieve the best results for all three PV models. TLABC achieves the best results for the SDM and PV module models. The other five algorithms, including CLPSO, BLPSO, ABC, GOTLBO and IJAVA, cannot obtain the best RMSE value on any $\mathrm{PV}$ models.

- Considering the mean and worst RMSE values, the proposed pSFS achieves the best results on two PV models, i.e., SDM and PV module. SFS gets the best result on the PV module model, but it performs worse than pSFS on SDM and DDM. This indicates that the chaotic elitist perturbation strategy improves the estimation accuracy of SFS. IJAVA is another competitive algorithm, as it attains the best result on DDM.

- The SD of RMSE can reflect the robustness of an algorithm in different runs. For this performance index, the proposed pSFS achieves the best results on the SDM and the PV module models. IJAVA gets the best SD result on DDM. It can also be observed that, pSFS achieves smaller SD values than SFS on all three PV models, which means the robustness of pSFS is enhanced by using the chaotic elitist perturbation strategy.

- According to the Wilcoxon rank sum test, the proposed pSFS performs significantly better than CLPSO, BLPSO, ABC, GOTLBO and TLABC on all three PV models. Compared with IJAVA and SFS, pSFS exhibits significantly better performance on the SDM and PV module.

- In terms of computational time, the eight algorithms use about 20 seconds for all three different PV models. Therefore, there are no clear differences among these algorithms.

Table 7 presents the Friedman rank values of the eight algorithms on the three problems PV parameter estimation problems. The proposed pSFS attains the best rank value (1.50), SFS the second(2.17), followed by IJAVA(2.67), TLABC(4.33), CLPSO(5.67), GOTLBO(6.33), ABC(6.33) and BLPSO(7.00).

Based on the above statistical comparisons and analysis, it can be concluded that the proposed pSFS achieves the overall best parameter estimation accuracy and robustness among the eight algorithms. The chaotic elitist perturbation strategy enhances the performance of the pSFS algorithm. Therefore, pSFS can be used as an accurate and robust method for PV parameter estimation problems with different diode models. 
Table 6: Statistical results of pSFS and the compared algorithms for different PV models

Table 7: Friedman rank values of theses algorithms on all three problems

\begin{tabular}{lcc}
\hline & Friedman rank & Final rank \\
\hline CLPSO & 5.67 & 5 \\
BLPSO & 7.00 & 8 \\
ABC & 6.33 & 6 \\
GOTLBO & 6.33 & 7 \\
TLABC & 4.33 & 4 \\
IJAYA & 2.67 & 3 \\
SFS & 2.17 & 2 \\
pSFS & 1.50 & 1 \\
\hline
\end{tabular}

\subsection{Comparisons of the convergence}

We also compare the convergence of the eight algorithms for the three PV models. Fig.12 plots the convergence curves in terms of average RMSE. From Fig.12, it can be observed that:

- IJAVA has the fastest convergence speed, followed by pSFS, SFS, TLABC and GOTLBO.

- The proposed pSFS has reasonably fast convergence speed. Meanwhile, it achieves high final estimation accuracy for all three PV models.

- pSFS converges faster than SFS for all three PV models. This indicates the chaotic elitist perturbation strategy accelerates its convergence speed.

- GOTLBO and TLABC have the similar convergence speed as that of pSFS, but their final estimation accuracy is worse than pSFS.

- The other three algorithms, BLPSO, CLPSO and ABC, converge relative slow. Also, their estimation accuracy are lower in comparison with other algorithms. 
Based on the above comparisions, it can be concluded that the proposed pSFS has a reasonably fast convergence speed, and its final convergence accuracy is the highest compared with all the other algorithms. In fact, an efficient seach process should balance the exploration and exploitation. In other words, too fast converge speed may make algorithms premature, while too solw converge speed may affect the final solution accuracy. For the multimodal PV parameter estimation problem, the proposed pSFS can keep an effective balance between global exploration and local exploitation. This is probably why pSFS can achieve high final estimation accuracy for all three PV models.

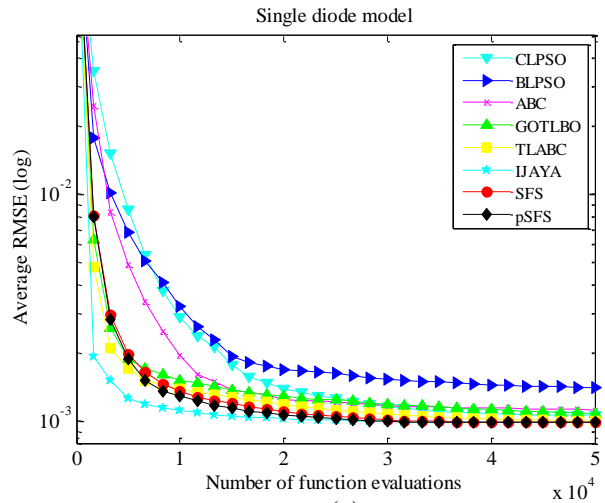

(a)

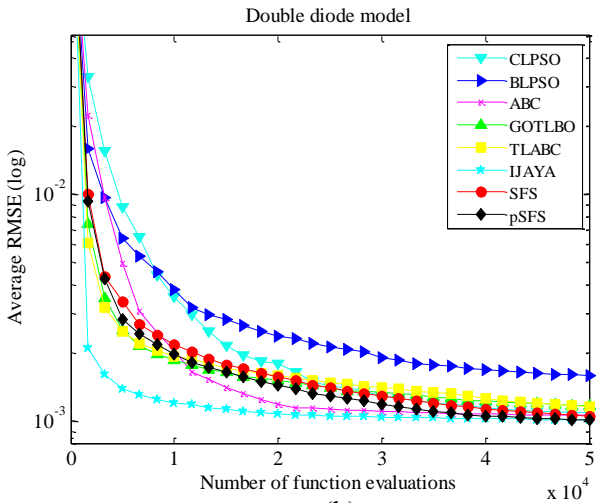

(b)

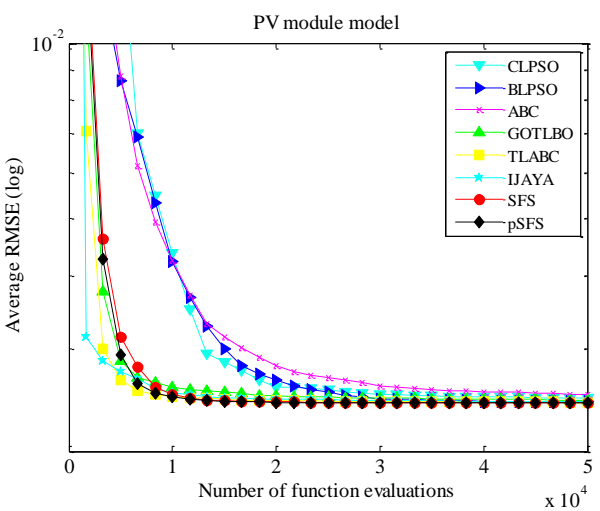

(c)

Figure 12: Convergence graphs of different algorithms for three PV models (a) SDM (b) DDM (c) PV module

\subsection{Parameter analysis}

To implement an efficient algorithm, it is also very important to analyze the tuning parameters. We analyze the impacts of the population size and the two Gaussian random walks on the performance of the proposed pSFS algorithm.

\subsubsection{Population size}

Table 8 shows the results of the proposed pSFS algorithm with population size $N P=10,20,30,40$ and 50 for three PV estimation problems. From the results listed in Table 8, it can be observed that:

- For the SDM, pSFS-NP30 yields the best performance for all four statistical results. Although pSFSNP20, pSFS-NP40, and pSFS-NP50 also provides the best results in terms of best RMSE, their results 
are not as good as pSFS-NP30 when comparing other performance indices.

- For the DDM, pSFS-NP30 obtains the best results in terms of worst and SD of RMSE.

- For the PV module model, pSFS-NP30 yields the best performance in terms of best, mean, worst and SD of RMSE. Meanwhile, pSFS-NP30 is the only algorithm which gets the minimal SD value.

These results indicate that pSFS with a moderate population size (i.e., pSFS-NP30) has the overall highest estimation accuracy and robustness. In fact, when the population size is too small, the population diversity is poor, which affects the global exploration and estimation accuracy. On the contrary, when the population size is too large, the algorithm needs more computational overheads (i.e., number of function evaluations) to converge. When the maximum number of function evaluations is fixed, too large population size may deteriorate the final performance. For the PV estimation problems, a moderate population size $N P=30$ is recommended for pSFS.

Table 8: Comparison of the pSFS algorithm with different population size for three PV models

\begin{tabular}{|c|c|c|c|c|c|}
\hline & & $\begin{array}{l}\text { RMSE } \\
\text { Min }\end{array}$ & Mean & $\operatorname{Max}$ & SD \\
\hline \multirow[t]{5}{*}{$\overline{\mathrm{SDM}}$} & pSFS-N10 & $9.8851 \mathrm{E}-04$ & $1.6630 \mathrm{E}-03$ & $3.6544 \mathrm{E}-03$ & $6.1567 \mathrm{E}-04$ \\
\hline & pSFS-N20 & $9.8602 \mathrm{E}-04$ & $1.0072 \mathrm{E}-03$ & $1.0854 \mathrm{E}-03$ & $3.2138 \mathrm{E}-05$ \\
\hline & pSFS-N30 & $9.8602 \mathrm{E}-04$ & $9.8602 \mathrm{E}-04$ & $9.8608 \mathrm{E}-04$ & $1.1063 \mathrm{E}-08$ \\
\hline & pSFS-N40 & 9.8602E-04 & $9.8603 \mathrm{E}-04$ & $9.8628 \mathrm{E}-04$ & $4.7319 \mathrm{E}-08$ \\
\hline & pSFS-N50 & $9.8602 \mathrm{E}-04$ & $9.8666 \mathrm{E}-04$ & $9.9449 \mathrm{E}-04$ & $1.6666 \mathrm{E}-06$ \\
\hline \multirow[t]{5}{*}{ DDM } & pSFS-N10 & $9.8774 \mathrm{E}-04$ & $1.8195 \mathrm{E}-03$ & $5.5085 \mathrm{E}-03$ & $1.0045 \mathrm{E}-03$ \\
\hline & pSFS-N20 & $9.8248 \mathrm{E}-04$ & $1.0946 \mathrm{E}-03$ & $2.2346 \mathrm{E}-03$ & $2.3917 \mathrm{E}-04$ \\
\hline & pSFS-N30 & $9.8255 \mathrm{E}-04$ & $1.0122 \mathrm{E}-03$ & 1.1930E-03 & 4.6733E-05 \\
\hline & pSFS-N40 & $9.8268 \mathrm{E}-04$ & $1.0118 \mathrm{E}-03$ & $1.3399 \mathrm{E}-03$ & $7.5648 \mathrm{E}-05$ \\
\hline & pSFS-N50 & $9.8291 \mathrm{E}-04$ & $1.0411 \mathrm{E}-03$ & $1.4825 \mathrm{E}-03$ & $1.0548 \mathrm{E}-04$ \\
\hline \multirow[t]{5}{*}{ PV module } & pSFS-N10 & $2.42570 \mathrm{E}-03$ & $1.22912 \mathrm{E}-02$ & $2.74251 \mathrm{E}-01$ & $4.94925 \mathrm{E}-02$ \\
\hline & pSFS-N20 & $2.42507 \mathrm{E}-03$ & $2.46508 \mathrm{E}-03$ & $3.17858 \mathrm{E}-03$ & $1.51147 \mathrm{E}-04$ \\
\hline & pSFS-N30 & 2.42507 E-03 & 2.42507 E-03 & 2.42507E-03 & $6.62209 \mathrm{E}-17$ \\
\hline & pSFS-N40 & $2.42507 \mathrm{E}-03$ & $2.42507 \mathrm{E}-03$ & $2.42508 \mathrm{E}-03$ & $2.53130 \mathrm{E}-10$ \\
\hline & pSFS-N50 & $2.42507 \mathrm{E}-03$ & $2.42507 \mathrm{E}-03$ & $2.42508 \mathrm{E}-03$ & $3.13602 \mathrm{E}-10$ \\
\hline
\end{tabular}

\subsubsection{Gaussian random walks}

Table 9 shows the results of the pSFS with two different Gaussian walks (GW). From Table 9, it can be found that:

- For the SDM, both pSFS-GW1 and pSFS-GW2 achieve the best results for the best RMSE. However, pSFS-GW1 yields better performance than pSFS-GW2 when considering the mean, worst and SD of RMSE.

- For the DDM, pSFS-GW2 achieves the better results for the best RMSE. pSFS-GW1 yields better performance than pSFS-GW2 when considering the mean, worst and SD of RMSE.

- For the PV module model, both pSFS-GW1 and pSFS-GW2 get the best results in terms of best, mean and worst RMSE. pSFS-GW2 obtains a smaller SD value than pSFS-GW1.

Our results indicate that there is no significant difference between pSFS-GW1 and pSFS-GW2 for the PV estimation problems. According to reports [28], pSFS-GW1 has a faster convergence speed and is more suitable for solving simple problems. pSFS-GW2 is more suitable for complicated problems, which needs more 
iteration number to converge. From the case study results in this work, pSFS-GW1 is recommended for the

Table 9: Comparison of the pSFS algorithm with two kinds of Gaussian walks

\begin{tabular}{|c|c|c|c|c|c|}
\hline & & $\begin{array}{c}\text { RMSE } \\
\text { Best }\end{array}$ & Mean & Worst & $\mathrm{SD}$ \\
\hline \multirow[t]{2}{*}{ SDM } & pSFS-GW1 & $9.8602 \mathrm{E}-04$ & $9.8602 \mathrm{E}-04$ & $9.8608 \mathrm{E}-04$ & $1.1063 \mathrm{E}-08$ \\
\hline & pSFS-GW2 & $9.8602 \mathrm{E}-04$ & $9.8608 \mathrm{E}-04$ & $9.8782 \mathrm{E}-04$ & $3.2842 \mathrm{E}-07$ \\
\hline \multirow[t]{2}{*}{ DDM } & pSFS-GW1 & $9.8255 \mathrm{E}-04$ & $1.0122 \mathrm{E}-03$ & 1.1930E-03 & 4.6733E-05 \\
\hline & pSFS-GW2 & 9.8249E-04 & $1.0113 \mathrm{E}-03$ & $1.4070 \mathrm{E}-03$ & $8.3206 \mathrm{E}-05$ \\
\hline \multirow[t]{2}{*}{ PV module } & pSFS-GW1 & 2.42507E-03 & 2.42507 E-03 & 2.42507E-03 & $6.62209 \mathrm{E}-17$ \\
\hline & pSFS-GW2 & 2.42507E-03 & 2.42507 E-03 & $2.42507 \mathrm{E}-03$ & $4.98125 \mathrm{E}-17$ \\
\hline
\end{tabular}

\section{Validation of pSFS with survey experimental data}

The above comparison studies demonstrate that pSFS has the highest estimation accuracy and robustness for parameter estimation for PV models. In this section, the practical use of pSFS is further examined by using experimental data from three different PV modules in the manufacturer data sheet: Thin-film ST40, Mono-crystalline SM55 and Multi-crystalline KC200GT [14].

The experimental $I-V$ data are extracted directly from the data sheet of five different irradiation levels at different temperature levels. The searching ranges for the five unknown parameters are: $I_{p h} \in\left[0,2 I_{s c}\right](\mathrm{A})$, $I_{s d} \in[0,100](\mu \mathrm{A}), R_{s} \in[0,2](\Omega), R_{s h} \in[0,5000](\Omega)$ and $a \in[1,4]$. The short circuit current $I_{s c}$ at nonstandard condition is calculated by Eq.(17):

$$
I_{s c}(G, T)=I_{s c \_S T C} \frac{G}{G_{S T C}}+\alpha\left(T-T_{S T C}\right)
$$

where $G$ and $T$ are the irradiation and the temperature levels, respectively; $G_{S T C}=1000 \mathrm{~W} / \mathrm{m}^{2}$ and $T_{S T C}=$ $25^{\circ} \mathrm{C} ; I_{\text {Sc_STC }}$ is short circuit current at standard test condition .

Table 10 and Table 11 present the optimal model parameters extracted by the proposed pSFS algorithm for three PV models under different irradiation and temperature levels, respectively. Furthermore, to verify the accuracy of the model parameters, the $I-V$ characteristics of the three different PV modules under different irradiation and temperature levels are plotted in Fig. 13 and Fig. 14, respectively.

From the results in Table 10 and Table 11, the optimal model parameters extracted by the proposed pSFS are in close with those by MPSO [14, and low RMSE values are achieved at different irradiation and temperature levels. From Fig. 13 and Fig. 14, the model curves calculated from the extracted model parameters match well with the experimental data under various environment conditions, i.e., at different irradiance and temperature levels. These observations indicate that the proposed pSFS approach can accurately extract the model parameters when the PV systems are under certain mismatch conditions such as partial shading. 
Table 10: Model parameters estimated by the pSFS algorithm for three PV modules at different irradiance and temperature of $25^{\circ} \mathrm{C}$

\begin{tabular}{|c|c|c|c|}
\hline Parameters & Thin-film ST40 & Mono-crystalline SM55 & Multi-crystalline $\mathrm{KC} 200 \mathrm{GT}$ \\
\hline \multicolumn{4}{|c|}{$G=1000 \mathrm{~W} / \mathrm{m}^{2}$} \\
\hline$I_{p h}(\mathrm{~A})$ & 2.67580 & 3.45010 & 8.21688 \\
\hline$I_{s d}(\mu \mathrm{A})$ & 1.52880 & 0.17115 & 0.00225 \\
\hline$R_{S}(\Omega)$ & 1.11323 & 0.32915 & 0.34376 \\
\hline$R_{s h}(\Omega)$ & 357.59844 & 483.90046 & 763.51258 \\
\hline$a$ & 1.50028 & 1.39575 & 1.07653 \\
\hline RMSE & $7.34099 \mathrm{E}-04$ & $1.14621 \mathrm{E}-03$ & $1.53933 \mathrm{E}-03$ \\
\hline \multicolumn{4}{|c|}{$G=800 \mathrm{~W} / \mathrm{m}^{2}$} \\
\hline$I_{p h}(\mathrm{~A})$ & 2.13801 & 2.76038 & 6.57104 \\
\hline$I_{s d}(\mu \mathrm{A})$ & 1.15810 & 0.14395 & 0.00098 \\
\hline$R_{S}(\Omega)$ & 1.12529 & 0.33759 & 0.35678 \\
\hline$R_{s h}(\Omega)$ & 332.88893 & 459.87849 & 754.80161 \\
\hline$a$ & 1.47315 & 1.38114 & 1.03680 \\
\hline RMSE & $7.73905 \mathrm{E}-04$ & $6.68579 \mathrm{E}-04$ & $1.64367 \mathrm{E}-03$ \\
\hline \multicolumn{4}{|c|}{$G=600 \mathrm{~W} / \mathrm{m}^{2}$} \\
\hline$I_{p h}(\mathrm{~A})$ & 1.60481 & 2.07090 & 4.93431 \\
\hline$I_{s d}(\mu \mathrm{A})$ & 1.44187 & 0.15551 & 0.00386 \\
\hline$R_{S}(\Omega)$ & 1.11261 & 0.33050 & 0.33734 \\
\hline$R_{s h}(\Omega)$ & 347.69469 & 450.06853 & 743.00159 \\
\hline$a$ & 1.49582 & 1.38753 & 1.10402 \\
\hline RMSE & $6.74036 \mathrm{E}-04$ & $8.23949 \mathrm{E}-04$ & $1.29767 \mathrm{E}-03$ \\
\hline \multicolumn{4}{|c|}{$G=400 \mathrm{~W} / \mathrm{m}^{2}$} \\
\hline$I_{p h}(\mathrm{~A})$ & 1.06754 & 1.38284 & 3.28785 \\
\hline$I_{s d}(\mu \mathrm{A})$ & 1.84875 & 0.10042 & 0.00149 \\
\hline$R_{S}(\Omega)$ & 1.08058 & 0.39665 & 0.35358 \\
\hline$R_{s h}(\Omega)$ & 362.51450 & 427.05044 & 752.08941 \\
\hline$a$ & 1.52445 & 1.35199 & 1.05504 \\
\hline RMSE & $6.30725 \mathrm{E}-04$ & $7.07608 \mathrm{E}-04$ & $1.42620 \mathrm{E}-03$ \\
\hline \multicolumn{4}{|c|}{$G=200 \mathrm{~W} / \mathrm{m}^{2}$} \\
\hline$I_{p h}(\mathrm{~A})$ & 0.53314 & 0.69151 & 1.64615 \\
\hline$I_{s d}(\mu \mathrm{A})$ & 1.42968 & 0.14641 & 0.00052 \\
\hline$R_{S}(\Omega)$ & 1.18572 & 0.28662 & 0.38111 \\
\hline$R_{s h}(\Omega)$ & 344.98324 & 448.21071 & 690.14660 \\
\hline$a$ & 1.49752 & 1.38066 & 1.00324 \\
\hline RMSE & $4.77201 \mathrm{E}-04$ & $3.20688 \mathrm{E}-04$ & $1.41847 \mathrm{E}-03$ \\
\hline
\end{tabular}

Table 11: Model parameters estimated by pSFS for three PV modules at different temperature and irradiance of $1000 \mathrm{~W} / \mathrm{m}^{2}$

\begin{tabular}{|c|c|c|c|c|c|c|c|}
\hline & Temperature & $I_{p h}(\mathrm{~A})$ & $I_{s d}(\mu \mathrm{A})$ & $R_{S}(\Omega)$ & $R_{s h}(\Omega)$ & $a$ & RMSE \\
\hline \multirow{4}{*}{ Thin-film ST40 } & $25^{\circ} \mathrm{C}$ & 2.67580 & 1.52880 & 1.11323 & 357.59844 & 1.50028 & 7.34099E-04 \\
\hline & $40^{\circ} \mathrm{C}$ & 2.68091 & 5.66610 & 1.12930 & 364.10973 & 1.47648 & $1.32141 \mathrm{E}-03$ \\
\hline & $55^{\circ} \mathrm{C}$ & 2.69197 & 18.68073 & 1.14959 & 295.02177 & 1.44978 & $1.82326 \mathrm{E}-03$ \\
\hline & $70^{\circ} \mathrm{C}$ & 2.69233 & 87.52185 & 1.12589 & 367.75322 & 1.54824 & $7.77718 \mathrm{E}-04$ \\
\hline \multirow{3}{*}{ Mono-crystalline SM55 } & $25^{\circ} \mathrm{C}$ & 3.45010 & 0.17115 & 0.32915 & 483.90046 & 1.39575 & $1.14621 \mathrm{E}-03$ \\
\hline & $40^{\circ} \mathrm{C}$ & 3.46914 & 1.14511 & 0.31310 & 533.06920 & 1.41784 & $3.78881 \mathrm{E}-03$ \\
\hline & $60^{\circ} \mathrm{C}$ & 3.49461 & 6.90950 & 0.31871 & 484.88387 & 1.40514 & $3.78039 \mathrm{E}-03$ \\
\hline \multirow{3}{*}{ Multi-crystalline KC200GT } & $25^{\circ} \mathrm{C}$ & 8.21688 & 0.00225 & 0.34376 & 763.51258 & 1.07653 & $1.53933 \mathrm{E}-03$ \\
\hline & $50^{\circ} \mathrm{C}$ & 8.29531 & 0.12595 & 0.33565 & 953.88989 & 1.11729 & $2.74651 \mathrm{E}-03$ \\
\hline & $75^{\circ} \mathrm{C}$ & 8.37766 & 1.63082 & 0.34250 & 790.55822 & 1.10148 & 4.47293E-03 \\
\hline
\end{tabular}




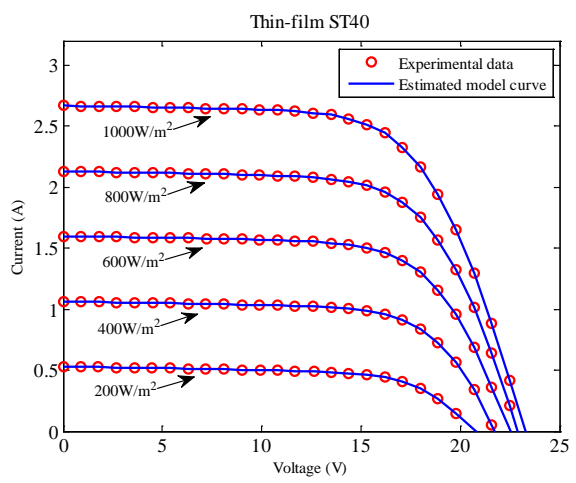

(a)

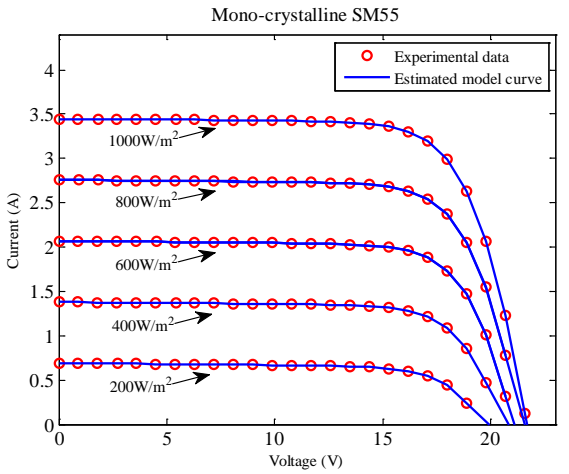

(b)

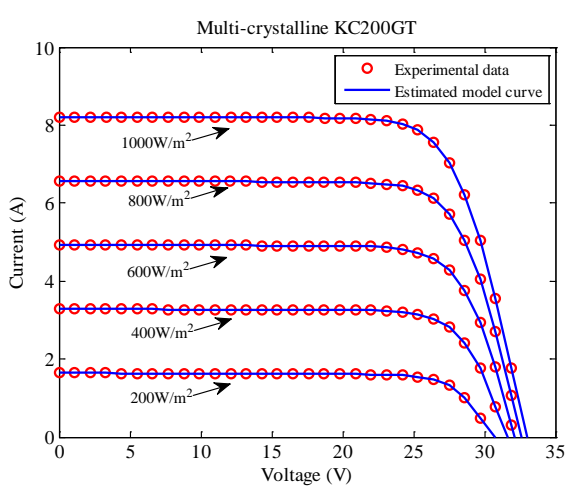

(c)

Figure 13: Comparisons between the experimental data and estimated data obtained by pSFS for three PV models at different irradiance, using real data from: (a) Thin-film ST40; (b) Mon-crystalline SM55; (c) Multi-crystalline KC200GT. 


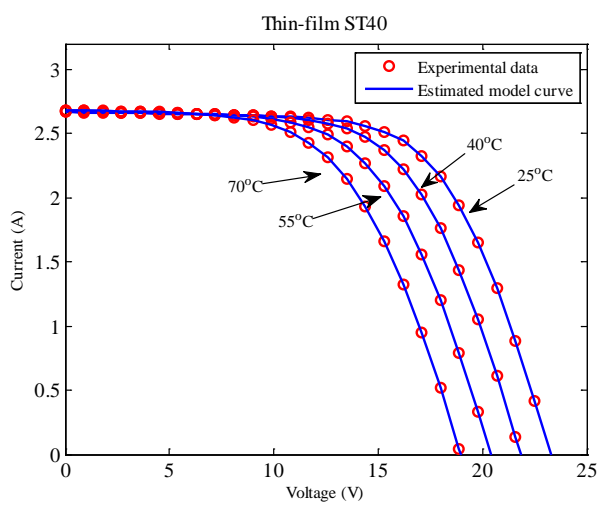

(a)

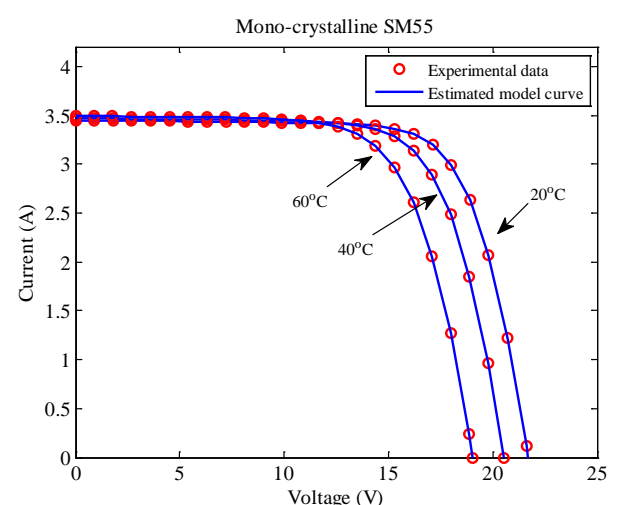

(b)

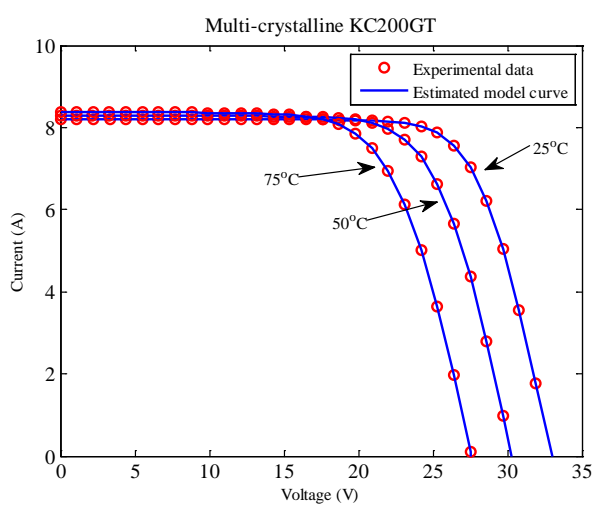

(c)

Figure 14: Comparisons between the experimental data and estimated data obtained by pSFS for three PV models at different temperatures, using real data from: (a) Thin-film ST40; (b) Mon-crystalline SM55; (c) Multi-crystalline KC200GT.

\section{Conclusion}

In this work, we have proposed a perturbed stochastic fractal search (pSFS) algorithm to accurately and robustly extract the PV model parameters. The proposed pSFS algorithm employs diffusion and updating processes inspired from random fractal properties. Meanwhile, a chaotic elitist perturbation strategy is also employed to perform self-adaptive local search around the best solution in each generation. The proposed pSFS algorithm is evaluated on PV parameter estimation problems with different diode models. Also, the performance of pSFS is compared with the basic SFS and six non-SFS algorithms. The following conclusions can be drawn from the numerical results.

- pSFS achieves high parameter estimation accuracy for different PV models. The statistical results demonstrate that pSFS has the best results in terms of the optimal, the mean and the worst RMSE values. Moreover, for the SDM and DDM, the absolute current error of pSFS is smaller than $0.3 \%$. For the PV module, the absolute current error of pSFS is smaller than $8 \%$.

- pSFS also has superiority in robustness compared with the recently developed algorithms including SFS, IJAVA, TLABC and GOTLBO. For the SDM, pSFS achieves the smallest SD value, the value of which 
is smaller than 1e-7. For the PV module, pSFS achieves the smallest SD value less than 1e-16.

- pSFS performs better than the other compared algorithms under comparison according to the Wilcoxon rank sum test.

- pSFS has a reasonably fast convergence speed, and keeps a good balance between global exploration and local exploitation during the searching process by employing two search operators.

- pSFS is robust to various environment conditions. The tests on three real PV modules at different irradiance and temperature levels show that pSFS achieves accurate results under all circumstances.

The proposed pSFS gains the above benefits mainly from two aspects. The first is the diffusion and updating processes from the basic SFS, which helps to achieve balance between global and local search. The second is the chaotic elitist perturbation strategy, which further enhances the estimation accuracy and robustness. In the future, we are interested in applying the pSFS algorithm to modeling of more complicated PV systems such as integral and fractional order dynamic PV system models. This novel algorithm can be applied to wider energy optimization problems, such as economic dispatch and unit commitment.

\section{Acknowledgement}

This work was supported by the UK Engineering and Physical Sciences Research Council (EPSRC) (Grant No. EP/R007497/1), the Natural Science Foundation of Jiangsu Province, China (Grant No. BK 20160540), and the National Natural Science Foundation of China (Grant No. 61806179).

\section{Appendix 1 Pseudocode of the pSFS algorithm}

\section{References}

[1] H. Chen, S. Jiao, A. A. Heidari, M. Wang, X. Chen, X. Zhao, An opposition-based sine cosine approach with local search for parameter estimation of photovoltaic models, Energy Conversion and Management 195 (2019) 927-942.

[2] S. Xu, Y. Wang, Parameter estimation of photovoltaic modules using a hybrid flower pollination algorithm, Energy Conversion and Management 144 (2017) 53-68.

[3] D. Alam, D. Yousri, M. Eteiba, Flower pollination algorithm based solar pv parameter estimation, Energy Conversion and Management 101 (2015) 410-422.

[4] K. Yu, J. Liang, B. Qu, Z. Cheng, H. Wang, Multiple learning backtracking search algorithm for estimating parameters of photovoltaic models, Applied Energy 226 (2018) 408-422.

[5] A. Ortiz-Conde, F. J. G. Sánchez, J. Muci, New method to extract the model parameters of solar cells from the explicit analytic solutions of their illuminated i-v characteristics, Solar Energy Materials and Solar Cells 90 (2006) 352-361. 


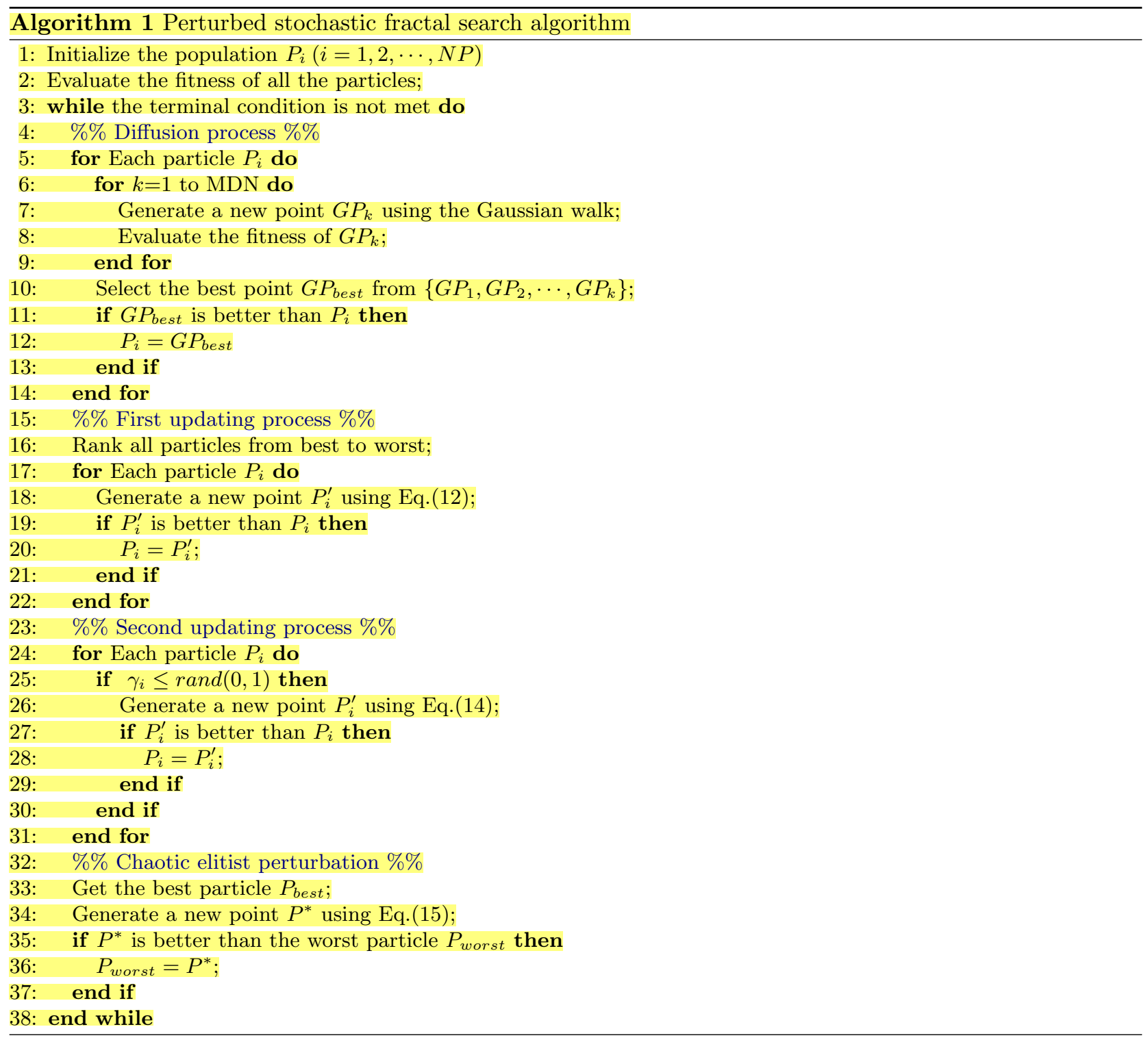

[6] T. Easwarakhanthan, J. Bottin, I. Bouhouch, C. Boutrit, Nonlinear minimization algorithm for determining the solar cell parameters with microcomputers, International Journal of Solar Energy 4 (1986) $1-12$.

[7] D. Chan, J. Phillips, J. Phang, A comparative study of extraction methods for solar cell model parameters, Solid-State Electronics 29 (1986) 329-337.

[8] X. Chen, H. Tianfield, , K. Li, Self-adaptive differential artificial bee colony algorithm for global optimization problems, Swarm and Evolutionary Computation 45 (2019) 70-91.

[9] B. Xu, X. Chen, L. Tao, Differential evolution with adaptive trial vector generation strategy and clusterreplacement-based feasibility rule for constrained optimization, Information Sciences 435 (2018) 240-262.

[10] Z. Zhu, L. Chen, C. Yuan, C. Xia, Global replacement-based differential evolution with neighbor-based memory for dynamic optimization, Applied Intelligence 48 (2018) 3280-3294. 
[11] A. A. Nagra, F. Han, Q.-H. Ling, S. Mehta, An improved hybrid method combining gravitational search algorithm with dynamic multi swarm particle swarm optimization, IEEE Access 7 (2019) 50388-50399.

[12] L. L. Jiang, D. L. Maskell, J. C. Patra, Parameter estimation of solar cells and modules using an improved adaptive differential evolution algorithm, Applied Energy 112 (2013) 185-193.

[13] A. R. Jordehi, Enhanced leader particle swarm optimisation (elpso): An efficient algorithm for parameter estimation of photovoltaic (pv) cells and modules, Solar Energy 159 (2018) 78-87.

[14] M. Merchaoui, A. Sakly, M. F. Mimouni, Particle swarm optimisation with adaptive mutation strategy for photovoltaic solar cell/module parameter extraction, Energy Conversion and Management 175 (2018) $151-163$.

[15] D. Yousri, D. Allam, M. Eteiba, P. N. Suganthan, Static and dynamic photovoltaic models parameters identification using chaotic heterogeneous comprehensive learning particle swarm optimizer variants, Energy Conversion and Management 182 (2019) 546-563.

[16] A. Askarzadeh, A. Rezazadeh, Parameter identification for solar cell models using harmony search-based algorithms, Solar Energy 86 (2012) 3241-3249.

[17] D. Oliva, E. Cuevas, G. Pajares, Parameter identification of solar cells using artificial bee colony optimization, Energy 72 (2014) 93-102.

[18] X. Chen, K. Yu, W. Du, W. Zhao, G. Liu, Parameters identification of solar cell models using generalized oppositional teaching learning based optimization, Energy 99 (2016) 170-180.

[19] D. Oliva, M. A. El Aziz, A. E. Hassanien, Parameter estimation of photovoltaic cells using an improved chaotic whale optimization algorithm, Applied Energy 200 (2017) 141-154.

[20] G. Xiong, J. Zhang, D. Shi, Y. He, Parameter extraction of solar photovoltaic models using an improved whale optimization algorithm, Energy Conversion and Management 174 (2018) 388-405.

[21] K. Yu, B. Qu, C. Yue, S. Ge, X. Chen, J. Liang, A performance-guided jaya algorithm for parameters identification of photovoltaic cell and module, Applied Energy 237 (2019) 241-257.

[22] R. Abbassi, A. Abbassi, A. A. Heidari, S. Mirjalili, An efficient salp swarm-inspired algorithm for parameters identification of photovoltaic cell models, Energy Conversion and Management 179 (2019) 362-372.

[23] T. S. Babu, J. P. Ram, K. Sangeetha, A. Laudani, N. Rajasekar, Parameter extraction of two diode solar pv model using fireworks algorithm, Solar Energy 140 (2016) 265-276.

[24] A. M. Beigi, A. Maroosi, Parameter identification for solar cells and module using a hybrid firefly and pattern search algorithms, Solar Energy 171 (2018) 435-446.

[25] X. Chen, B. Xu, C. Mei, Y. Ding, K. Li, Teaching-learning-based artificial bee colony for solar photovoltaic parameter estimation, Applied Energy 212 (2018) 1578-1588. 
[26] X. Chen, K. Yu, Hybridizing cuckoo search algorithm with biogeography-based optimization for estimating photovoltaic model parameters, Solar Energy 180 (2019) 192-206.

[27] L. Wu, Z. Chen, C. Long, S. Cheng, P. Lin, Y. Chen, H. Chen, Parameter extraction of photovoltaic models from measured iv characteristics curves using a hybrid trust-region reflective algorithm, Applied Energy 232 (2018) 36-53.

[28] H. Salimi, Stochastic fractal search: a powerful metaheuristic algorithm, Knowledge-Based Systems 75 (2015) 1-18.

[29] M. A. Mellal, E. Zio, A penalty guided stochastic fractal search approach for system reliability optimization, Reliability Engineering \& System Safety 152 (2016) 213-227.

[30] E. Çelik, Incorporation of stochastic fractal search algorithm into efficient design of pid controller for an automatic voltage regulator system, Neural Computing and Applications (2018) 1-12.

[31] J. Lin, Z.-J. Wang, Multi-area economic dispatch using an improved stochastic fractal search algorithm, Energy 166 (2019) 47-58.

[32] W. Gong, Z. Cai, Parameter extraction of solar cell models using repaired adaptive differential evolution, Solar Energy 94 (2013) 209-220.

[33] K. Yu, J. Liang, B. Qu, X. Chen, H. Wang, Parameters identification of photovoltaic models using an improved jaya optimization algorithm, Energy Conversion and Management 150 (2017) 742-753.

[34] D. H. Muhsen, A. B. Ghazali, T. Khatib, I. A. Abed, Parameters extraction of double diode photovoltaic modules model based on hybrid evolutionary algorithm, Energy conversion and management 105 (2015) $552-561$.

[35] X. Gao, Y. Cui, J. Hu, G. Xu, Z. Wang, J. Qu, H. Wang, Parameter extraction of solar cell models using improved shuffled complex evolution algorithm, Energy Conversion and Management 157 (2018) 460-479.

[36] S. Li, W. Gong, X. Yan, C. Hu, D. Bai, L. Wang, L. Gao, Parameter extraction of photovoltaic models using an improved teaching-learning-based optimization, Energy Conversion and Management 186 (2019) 293-305.

[37] J. J. Liang, A. K. Qin, P. N. Suganthan, S. Baskar, Comprehensive learning particle swarm optimizer for global optimization of multimodal functions, IEEE transactions on evolutionary computation 10 (2006) 281-295.

[38] X. Chen, H. Tianfield, C. Mei, W. Du, G. Liu, Biogeography-based learning particle swarm optimization, Soft Computing 21 (2017) 7519-7541.

[39] D. Karaboga, B. Basturk, A powerful and efficient algorithm for numerical function optimization: artificial bee colony (abc) algorithm, Journal of global optimization 39 (2007) 459-471. 


\title{
Perturbed stochastic fractal search for solar PV parameter estimation
}

\begin{abstract}
Following the widespread use of solar energy all over the world, the design of high quality photovoltaic (PV) cells has attracted strong research interests. To properly evaluate, control and optimize solar PV systems, it is crucial to establish a reliable and accurate model, which is a challenging task due to the presence of non-linearity and multi-modality in the PV systems. In this work, a new meta-heuristic algorithm (MHA), called perturbed stochastic fractal search (pSFS), is proposed to estimate the PV parameters in an optimization framework. The novelty lies in two aspects: (i) employ its own searching operators, i.e., diffusion and updating, to achieve a balance between the global exploration and the local exploitation; and (ii) incorporate a chaotic elitist perturbation strategy to improve the searching performance. To examine the effectiveness of pSFS, this method is applied to solve three PV estimation problems for different PV models, including single diode, double diode and PV modules. Experimental results and statistical analysis show that the proposed pSFS has improved estimation accuracy and robustness compared with several other algorithms recently developed.
\end{abstract}

Keywords: Photovoltaic (PV) modeling, parameters estimation, stochastic fractal search, chaotic elitist perturbation

\section{Introduction}

Solar energy is considered to be a promising renewable energy because of its affluent availability and cleanliness. Solar photovoltaic (PV) systems can convert solar energy into electricity and supply power. It has been widely used for several decades and the rapid growth is still continuing [1].

Mathematical models are important for control, optimization and assessment of solar PV systems [2]. Several PV models have been developed, among which the single diode model (SDM) and the double diode model (DDM) are most widely used [3. The prediction quality of a PV model largely depends on the extracted model parameters. Therefore, accurate and robust estimation of model parameters is crucial for PV modeling.

\subsection{Literature review}

PV parameter estimation is often formulated as an optimization problem to minimize the residual errors statistically. Due to the non-linearity inherent in the dynamics and the noise involved in the experimental

\footnotetext{
*Corresponding author

Email addresses: xuchen@ujs.edu.cn (Xu Chen), hong.yue@strath.ac.uk (Hong Yue), yukunjie@zzu.edu.cn (Kunjie Yu)
} 


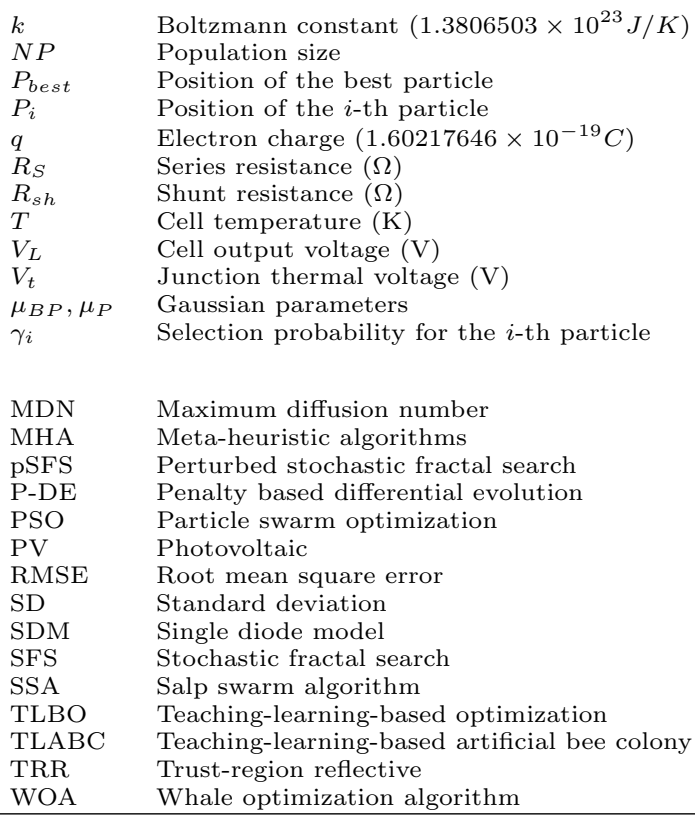

current-voltage $(I-V)$ data, PV parameter estimation is usually a multimodal problem with multiple local optimums [4. Deterministic techniques have been proposed for solving this problem, including the Lambert W-functions [5], the Newton-Raphson method [6], and the iterative curve fitting [7]. Most of the deterministic techniques are based on the gradient information. They show powerful local search abilities, but likely fall into local optimums. In addition, deterministic techniques require strict conditions such as the differentiability and convexity, which restricts their wide applications.

More recently, meta-heuristic algorithms (MHAs) have been developed and employed for PV parameters estimation problems. Inspired by natural phenomena, MHAs have advantages in solving complex global optimization problems [8, 9], and have no requirements such as continuity, differentiability or convexity for the optimization problems 10, 11. There are three groups of MHAs used for parameter estimation, i.e., the classic MHAs, the latest MHAs and the hybrid MHAs.

The classic MHAs include algorithms such as differential evolution (DE), particle swarm optimization (PSO), harmony search (HS), artificial bee colony (ABC) and teaching-learning-based optimization (TLBO). DE has a simple structure and exhibits good accuracy, but its optimization results highly depend on two control parameters, namely the scaling factor and the crossover rate. In [12], the fitness information was used to tune the control parameters, and an improved adaptive DE (IADE) was proposed for estimating parameters of PV models. PSO is also simple to implement and has fast convergence speed, but it often suffers from premature convergence. To overcome this drawback, several PSO variants including the enhanced leader PSO (ELPSO) [13, the adaptive mutation strategy (MPSO) [14] and the chaotic heterogeneous comprehensive learning PSO (CHCLPSO) [15, have been developed and applied to extract the PV model parameters. In [16], three HS variants were used to determine the unknown parameters of solar cell models. The simulation studies show that HS algorithms achieve improved results compared to the simulated annealing and the pattern search. In [17], artificial bee colony (ABC) algorithm was used to identify the parameters of two solar cells. It is observed that 
ABC has good search ability for multimodal objective functions compared with HS, PSO, genetic algorithm and bacterial foraging algorithm. In [18, the generalized oppositional TLBO (GOTLBO) algorithm was proposed to identify the parameters of solar cell models. By using the generalized opposition-based learning, the GOTLBO algorithm accelerates the convergence speed compared to the basic TLBO.

There is not a single classic MHA that is competent for all optimization problems. The latest MHAs are used for PV parameter estimation. In [19, the whale optimization algorithm (WOA) was proposed for PV parameters estimation, in which the chaotic map was used to automatically adjust the internal parameters of WOA. This helps to avoid local optimum and also improves the convergence rate. In 20, two prey searching strategies were embedded into WOA, which overcomes the problem of premature convergence, and the improved WOA was applied to estimate the model parameters of two practical PV power stations. Alam et al. 3. proposed a flower pollination algorithm (FPA) based method for PV parameters estimation method to improve the convergence performance. Improved JAYA algorithms were proposed to accurately and reliably identify the parameters of several PV models [4, 21. However, JAYA algorithms need relatively large number of iterations to converge. Several other latest MHAs including the salp swarm algorithm (SSA) 22] and the fireworks algorithm (FWA) 23] were also utilized to extract PV model parameters, and achieve competitive performance in the reported case studies.

Hybrid MHAs combine advantages from more than one MHA, thus provide more accurate parameters than the individual algorithms. Several hybrid MHAs were also developed for PV parameter estimation problems, including the hybrid firefly algorithm and pattern search (HFAPS) 24, the teaching-learning-based artificial bee colony (TLABC) 25, the biogeography-based heterogeneous cuckoo search (BHCS) 26, and the trustregion reflective artificial bee colony (ABC-TRR) [27].

\subsection{Contribution}

The above short literature review shows that parameter estimation of PV models is still a challenging task that requires more effective and efficient tools. Recent development suggests that MHAs have good potential for parameter estimation of PV models. In this work, we will explore a particular MHA, the stochastic fractal search (SFS), for PV modeling.

SFS is an MHA which uses the diffusion and update processes based on random fractal properties [28]. This algorithm is developed to overcome the weaknesses of MHAs such as premature convergence and low robustness. SFS has been applied in solving several real-world problems, such as system reliability optimization [29], PID controller design for an automatic voltage regulator system [30, and electric power economic dispatch [31].

Inspired by the recent progress of SFS in applications, in this study, we investigate the further development of SFS for the challenging PV parameter estimation problems. Specifically, we propose a perturbed stochastic fractal search (pSFS) algorithm, which employs the diffusion and updating operators in searching. Moreover, a simple but efficient chaotic elitist perturbation is incorporated into the pSFS, which drives the local search around the best solution during the searching process. This designed perturbation can further improve the solution accuracy and robustness. The proposed pSFS is applied to solve three PV parameter estimation problems with different diode models, and the results are compared with several recently-developed MHAs to 
verify its performance.

The contributions of this paper are listed as follows:

(1) A new perturbed SFS (pSFS) approach is proposed for solving PV parameter estimation problems.

(2) A chaotic elitist perturbation strategy is implemented to improve the search efficiency.

(3) pSFS is applied to three PV parameter estimation problems with different diode models, as well as three PV modules using survey data.

(4) By comparing with the recently-developed algorithms, the superiority of pSFS in solution accuracy and robustness are demonstrated.

\subsection{Paper organization}

The remaining of this paper is organized as follows. In Section 2, three PV parameter estimation problems are defined for SDM, DDM and PV module, respectively. The basic SFS and the proposed pSFS algorithms are presented in Section 3. Comparisons are made in Section 4 between the pSFS and several recently-developed algorithms. In Section 5, the practical use of pSFS is examined by using surveyed experimental data from three different PV modules. Finally, conclusions are given in Section 6.

\section{Problem statement}

This section presents the mathematical formulation of PV parameter estimation problems with three different diode models, i.e., SDM, DDM, and PV module models.

\subsection{Single diode model}

The equivalent electric circuit of a SDM is shown in Fig.1. In SDM, the output current $I_{L}$ is calculated as follows [32, 33]:

$$
I_{L}=I_{p h}-I_{d}-I_{s h}=I_{p h}-I_{s d} \cdot\left[\exp \left(\frac{V_{L}+R_{S} \cdot I_{L}}{a \cdot V_{t}}\right)-1\right]-\frac{V_{L}+R_{S} \cdot I_{L}}{R_{s h}}
$$

where $I_{p h}, I_{d}$ and $I_{s h}$ are the photo-generated current, diode current and shunt resistor current, respectively; $V_{L}$ is the cell output voltage; $I_{s d}$ is the reverse saturation current; $R_{S}$ and $R_{s h}$ are the series resistance and shunt resistance, respectively; $a$ is the diode ideality constant; and $V_{t}$ is the junction thermal voltage calculated as follows:

$$
V_{t}=\frac{k T}{q}
$$

where $k=1.3806503 \times 10^{23} \mathrm{~J} / \mathrm{K}$ is the Boltzmann constant, $q=1.60217646 \times 10^{-19} \mathrm{C}$ is the electron charge, and $T$ is the cell temperature $(K)$. 


\subsection{Double diode model}

The equivalent electric circuit of the DDM is shown in Fig.2. In DDM, the cell output current $I_{L}$ can be calculated as follows 32,33 :

$$
\begin{aligned}
I_{L} & =I_{p h}-I_{d 1}-I_{d 2}-I_{s h} \\
& =I_{p h}-I_{s d 1} \cdot\left(\exp \left(\frac{V_{L}+I_{L} R_{S}}{a_{1} V_{t}}\right)-1\right)-I_{s d 2} \cdot\left(\exp \left(\frac{V_{L}+I_{L} R_{S}}{a_{2} V_{t}}\right)-1\right)-\frac{V_{L}+I_{L} R_{s}}{R_{s h}}
\end{aligned}
$$

where $I_{d 1}$ and $I_{d 2}$ denote the first and the second diode currents, respectively; $I_{s d 1}$ and $I_{s d 2}$ denote the diffusion and saturation currents, respectively; $a_{1}$ and $a_{2}$ are the diffusion and recombination diode ideality factors, respectively.

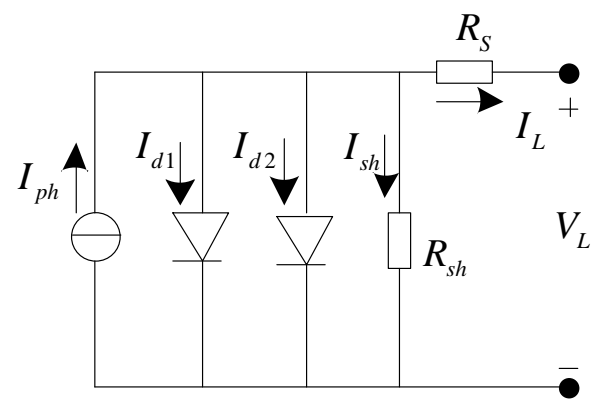

Figure 2: Equivalent electric circuit of double diode model

\subsection{PV module model}

The PV module model consists of $N_{s} \times N_{p}$ solar cells connected in series and/or in parallel as shown in Fig.3. The cell output current $I_{L}$ can be formulated as follows [32, 33]:

$$
I_{L}=N_{p} \cdot I_{p h}-N_{p} \cdot I_{s d} \cdot\left[\exp \left(\frac{V_{L} / N_{S}+R_{S} \cdot I_{L} / N_{p}}{a \cdot V_{t}}\right)-1\right]-\frac{N_{p} \cdot V_{L} / N_{S}+R_{S} \cdot I_{L}}{R_{s h}}
$$

where $N_{p}$ and $N_{s}$ are the numbers of solar cells in parallel and in series, respectively. 


\subsection{Objective function of $P V$ estimation problem}

In order to extract the unknown parameters of PV models based on the experimental data, the PV parameter estimation is converted into an optimization problem. The objective function is defined as the overall root mean square error (RMSE) between experimental data and simulated data as follows [18, 34]:

$$
\operatorname{RMSE}(\boldsymbol{x})=\sqrt{\frac{1}{N} \sum_{k=1}^{N} f_{k}\left(V_{L}, I_{L}, \boldsymbol{x}\right)^{2}}
$$

where $N$ is the number of experimental data, and $\boldsymbol{x}$ is the vector of unknown model parameters.

For the SDM,

$$
\begin{gathered}
f_{k}\left(V_{L}, I_{L}, \boldsymbol{x}\right)=I_{p h}-I_{s d}\left(\exp \left(\frac{V_{L}+I_{L} R_{S}}{a V_{t}}\right)-1\right)-\frac{V_{L}+I_{L} R_{s}}{R_{s h}}-I_{L} \\
\boldsymbol{x}=\left\{I_{p h}, I_{s d}, R_{S}, R_{s h}, a\right\}
\end{gathered}
$$

For the DDM,

$$
\begin{gathered}
f_{k}\left(V_{L}, I_{L}, \boldsymbol{x}\right)=I_{p h}-I_{s d 1}\left(\exp \left(\frac{V_{L}+I_{L} R_{S}}{a_{1} V_{t}}\right)-1\right)-I_{s d 2}\left(\exp \left(\frac{V_{L}+I_{L} R_{S}}{a_{2} V_{t}}\right)-1\right)-\frac{V_{L}+I_{L} R_{s}}{R_{s h}}-I_{L} \\
\boldsymbol{x}=\left\{I_{p h}, I_{s d 1}, I_{s d 2}, R_{S}, R_{s h}, a_{1}, a_{2}\right\}
\end{gathered}
$$

For the PV module model,

$$
\begin{gathered}
f_{k}\left(V_{L}, I_{L}, \boldsymbol{x}\right)=N_{p} I_{p h}-N_{p} I_{s d}\left[\exp \left(\frac{V_{L} / N_{S}+R_{S} I_{L} / N_{p}}{a V_{t}}\right)-1\right]-\frac{N_{p} V_{L} / N_{S}+R_{S} I_{L}}{R_{s h}}-I_{L} \\
\boldsymbol{x}=\left\{I_{p h}, I_{s d}, R_{S}, R_{s h}, a\right\}
\end{gathered}
$$




\section{Perturbed stochastic fractal search for $\mathbf{P V}$ model estimation}

\subsection{Stochastic fractal search}

SFS is a recently developed MHA proposed by Salimi [28, taking inspiration from the natural growth phenomenon of random fractal. The SFS algorithm mainly uses two processes namely diffusion and updating to improve the searching. In the diffusion process, each particle (i.e., candidate solution) diffuses around its own location, and carries out the exploitation task. By contrast, in the updating process, each particle is updated according to the location of other particles, and this process leads to exploration properties. The two processes of SFS are shown in Fig. 4.
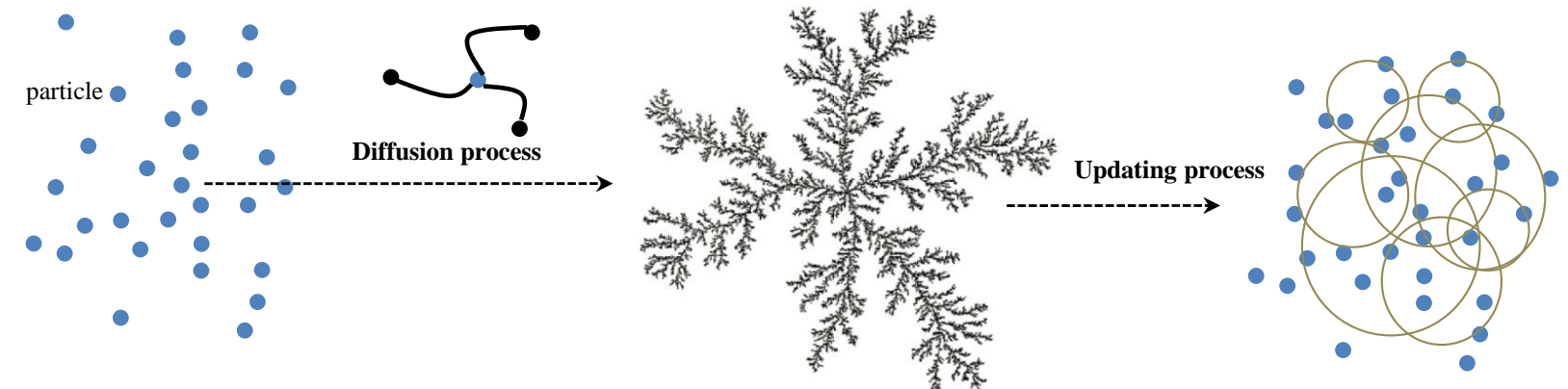

Figure 4: The main procedure of SFS algorithm

The diffusion process uses Gaussian random walks to generate points around each particle until a predetermined maximum diffusion number $(\mathrm{MDN})$ is reached. There are two types of Gaussian walks in the diffusion process, which are described as follows:

$$
\begin{gathered}
G W_{1}=\operatorname{Gaussian}\left(\mu_{B P}, \delta\right)+\left(\operatorname{rand}(0,1) \times P_{\text {best }}-\operatorname{rand}(0,1) \times P_{i}\right) \\
G W_{2}=\operatorname{Gaussian}\left(\mu_{P}, \delta\right)
\end{gathered}
$$

where $P_{i}$ and $P_{b e s t}$ are the positions of the $i$-th and the best particles, respectively; $i=1,2, \cdots, N P, N P$ is the population size; $\operatorname{rand}(0,1)$ is a random number generated within $[0,1]$. Gaussian parameters $\mu_{B P}$ and $\mu_{P}$ are equal to $P_{\text {best }}$ and $P_{i}$, respectively. The standard deviation $\delta$ is dynamically adjusted based on the number of the generation $G$ :

$$
\delta=\left|\frac{\log (G)}{G} \times\left(P_{i}-P_{\text {best }}\right)\right|
$$

The update process employs two statistical procedures to undertake the exploration in SFS. In the first updating process, each particle position is updated as follows:

$$
P_{i}^{\prime}(j)=\left\{\begin{array}{l}
P_{r 1}(j)-\operatorname{rand}(0,1) \times\left(P_{r 2}(j)-P_{i}(j)\right) \quad \text { if } \gamma_{i}<\operatorname{rand}(0,1) \\
P_{i}(j) \quad \text { otherwise }
\end{array}\right.
$$

where $j \in\{1,2, \cdots, D\}$ is the index for each optimization variable; $D$ is the number of optimization variables; 
$P_{i}^{\prime}$ is the new position of $P_{i} ; P_{r 1}$ and $P_{r 2}$ are the positions of two randomly selected particles; $\gamma_{i}$ is the selection probability for particle $P_{i}$ that can be calculated as:

$$
\gamma_{i}=1-\frac{\operatorname{rank}\left(P_{i}\right)}{N P}
$$

where $\operatorname{rank}\left(P_{i}\right)$ is the fitness order from the best to the worst of the $i$-th particles in the population. Obviously, the worse particle has a smaller $\gamma_{i}$ undergoing the first update process.

In the second updating process, the probability $\gamma_{i}$ is recalculated and used to determine whether a particle $P_{i}$ should be updated as follows:

$$
P_{i}^{\prime}(j)= \begin{cases}P_{i}(j)-\operatorname{rand}(0,1) \times\left(P_{r 1}^{\prime}(j)-P_{\text {best }}(j)\right) & \text { if } \operatorname{rand}(0,1)<0.5 \\ P_{i}(j)+\operatorname{rand}(0,1) \times\left(P_{r 1}^{\prime}(j)-P_{r 2}^{\prime}(j)\right) & \text { otherwise }\end{cases}
$$

where $P_{r 1}^{\prime}$ and $P_{r 2}^{\prime}$ are the positions of two randomly selected particles.

\subsection{Perturbed stochastic fractal search}

\subsubsection{Chaotic elitist perturbation strategy}

During the search process of SFS, the best solution found is recorded in each generation. Its quality affects both the search process and the final solution. Chaotic elitist perturbation strategy is introduced to perform adaptive local search around the best solution as follows:

$$
P^{*}(j)=\left\{\begin{array}{l}
P_{\text {best }}(j)+\operatorname{rand}(0,1) \cdot\left(2 z_{k}-1\right) \quad \text { if } \operatorname{rand}(0,1)<1-F E S / F E S_{\max } \\
P_{\text {best }}(j) \text { otherwise }
\end{array}\right.
$$

where the logistic map $z_{k}=4 z_{k-1}\left(1-z_{k-1}\right)$ is used to generate the $k$-th chaotic iteration value, with its initial value $z_{0}$ randomly generated within $[0,1] ; F E S$ and $F E S_{\max }$ are the current and maximum number of function evaluations. The new solution $P^{*}$ is compared with the worst solution $P_{\text {worst }}$ in current population, and the better one is selected for the next generation.

According to Eq. (18), the chaotic elitist perturbation strategy can perform a self-adaptive perturbation during the optimization process. To be specific, the value of FES is relative small in the early search stage, thus more perturbations will be added to the best solution, which is useful for global exploration. By contrast, the value of $F E S$ is close to 1 in the latter search stage, therefore more information will be inherited from the best solution, which is beneficial for local exploitation.

\subsubsection{Algorithmic framework}

The flowchart of the proposed pSFS algorithm is presented in Fig.5. A more detailed description of pSFS using the pseudocode is presented in Appendix 1. It can be seen that, the proposed pSFS takes four searching stages, including the diffusion process, the first and second updating processes, and the chaotic elitist perturbation. These four stages have different roles in the searching process. The diffusion process focuses on 


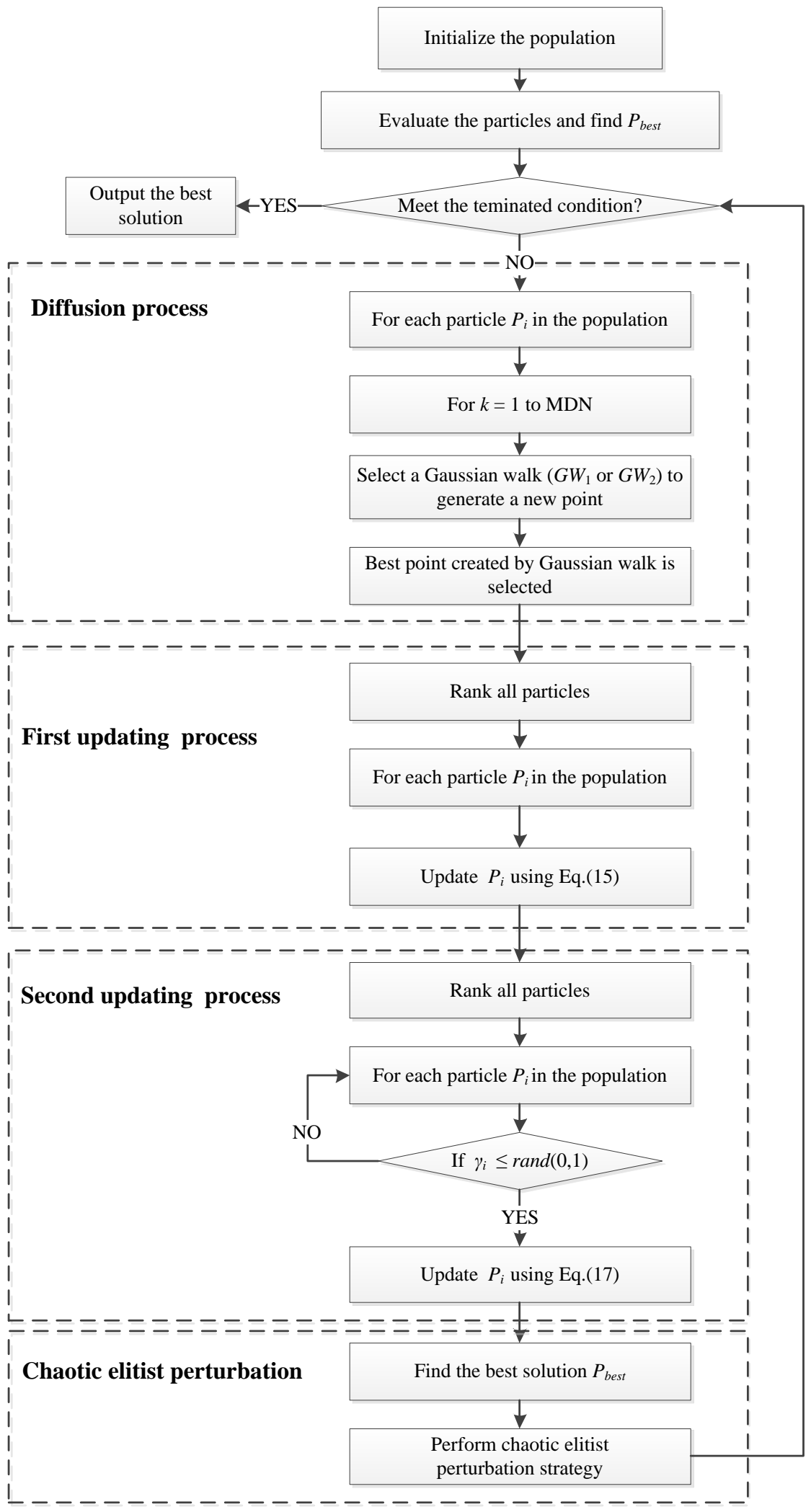

Figure 5: Flowchart of the pSFS algorithm 
local exploitation, the two updating processes focus on global exploration, and the chaotic elitist perturbation strategy performs adaptive local search to further improve the solution accuracy and robustness.

The computational complexity of pSFS mainly includes: (a) the time for the diffusion process $T_{d i}$, (b) the time for the first updating process $T_{u p 1}$, (c) the time for the second updating process $T_{u p 2}$, and (d) the time for the chaotic elitist perturbation strategy $T_{p e}$. The total computational complexity of pSFS can be represented by a time metric as follows:

$$
\begin{aligned}
T_{p S F S}= & \left(T_{d i}+T_{u p 1}+T_{u p 2}+T_{p e}\right) \cdot G_{\max } \\
= & (O(N P \cdot M D N \cdot D)+O(N P \cdot \log (N P)+N P \cdot D) \\
& +O(N P \cdot \log (N P)+N P \cdot D)+O(D)) \cdot G_{\max } \\
= & O(M D N \cdot D+2 \log (N P)+2 D) \cdot N P \cdot G_{\max } \\
= & O(M D N \cdot D+2 \log (N P)+2 D) \cdot F E S_{\max }
\end{aligned}
$$

where $D$ is the problem dimension, $N P$ is the population size, $M D N$ is the maximum diffusion number, $G_{m a x}$ is the maximum number of generations, and $F E S_{\max }$ is the maximum number of function evaluations.

\section{Results and analysis}

The proposed pSFS algorithm is evaluated by solving three PV parameter estimation problems as described in Section 2. The experimental current-voltage data of the SDM and DDM are measured from a RTC France solar cell (under $1000 \mathrm{~W} / \mathrm{m}^{2}$ at $33{ }^{\circ} \mathrm{C}$ ) and are taken from [6]. The experimental current-voltage data of the PV module are from Photowatt-PWP201 module (under $1000 \mathrm{~W} / \mathrm{m}^{2}$ at $45{ }^{\circ} \mathrm{C}$ ) [6]. The searching ranges for the model parameters are listed in Table 1, which are the same as those in [4, 35, 36]. This will ensure comparisons are made under the same system settings.

The pSFS is compared with seven recently-developed algorithms: basic SFS [28], CLPSO (comprehensive learning PSO) 37], BLPSO (biogeography-based learning PSO) [38, ABC (artificial bee colony) [39], GOTLBO (generalized oppositional TLBO) [18, TLABC (teaching-learning-based ABC) [25], and IJAYA (improved JAYA) 33. These algorithms show good performance for PV parameter estimation, therefore chosen for comparison in this study.

The parameter settings for these algorithms under comparison are given in Table 2, mainly taken from their corresponding literature. The tuning parameters for the proposed pSFS are also given in Table 2 . The maximum number of function evaluations is set to be $F E S_{\max }=50000$ for all three PV parameter estimation problems [25, 4, 36]. All the algorithms are coded in MATLAB and run 30 times independently to obtain the statistical results. The experiments are carried out on a DELL computer with a Core i5-4460 processor and 8G RAM.

\subsection{Comparisons based on the best results \\ 4.1.1. Results on the single diode mode}

We first compare the proposed pSFS with the other seven algorithms on the SDM. There are five unknown parameters to be estimated in the SDM model. Table 3 presents the comparison results for all these algorithms. 


\begin{tabular}{ccccc}
\multicolumn{2}{c}{ Table 1: Parameter boundaries of three PV models. } \\
\hline Parameter & \multicolumn{2}{c}{ SDM/DDM } & \multicolumn{2}{c}{ PV module } \\
& Lower & Upper & Lower & Upper \\
\hline$I_{p h}(\mathrm{~A})$ & 0 & 1 & 0 & 2 \\
$I_{s d}, I_{s d 1}, I_{s d 2}(\mu \mathrm{A})$ & 0 & 1 & 0 & 50 \\
$R_{S}(\Omega)$ & 0 & 0.5 & 0 & 2 \\
$R_{s h}(\Omega)$ & 0 & 100 & 0 & 2000 \\
$a, a_{1}, a_{2}$ & 1 & 2 & 1 & 50 \\
\hline
\end{tabular}

Table 2: Parameter settings for pSFS and the other algorithms.

\begin{tabular}{lll}
\hline Algorithm & Year & Parameter settings \\
\hline CLPSO & 2006 & $N P=40, w=0.9 \sim 0.2, c=1.496, m=5$ \\
BLPSO & 2017 & $N P=40, w=0.9 \sim 0.2, c=1.496, I=E=1$ \\
ABC & 2007 & $N P=50$, limit $=200$ \\
GOTLBO & 2016 & $N P=50$, Jr $=0.3$ \\
TLABC & 2018 & $N P=50$, limit $=200, F=\operatorname{rand}(0,1)$ \\
IJAYA & 2017 & $N P=20$ \\
SFS & 2015 & $N P=30, M D N=1$, Gaussian walk $G W_{1}$ \\
pSFS & proposed & $N P=30, M D N=1$, Gaussian walk $G W_{1}$ \\
\hline
\end{tabular}

The extracted model parameters are also presented. Note that, the results reported in Table 3 are the best RMSE values among 30 independent runs. The statistical results will be compared in Section 4.2.

From the results in Table 3, the proposed pSFS, together with SFS and TLABC achieve the best RMSE value (i.e., 9.8602E-4). The RMSE reflects the estimation accuracy of the algorithms. Thus, the highest estimation accuracy has been achieved by pSFS, SFS and TLABC. IJAVA achieves the RMSE value of 9.8603E04, followed by GOTLBO (9.8658E-04) and ABC (9.8815E-04). The performance of CLPSO and BLPSO stays on the lower end of the comparison, and their RMSE values are 9.9207E-04 and 1.1239E-03, respectively.

The extracted parameters of pSFS are used to plot the $I-V$ and $P-V$ curves. As shown in Fig.6, both $I-V$ and $P-V$ characteristics curves demonstrate that the estimated model curves are highly consistent with the experimental data. Besides, Fig.7 plots the individual absolute current error (IACE) and individual absolute power error (IAPE) over the whole voltage range. The maximal IACE value is smaller than 2.5E-3, and the maximal IAPE value is smaller than 1.5E-3. All these observations demonstrate the high estimation accuracy of the pSFS algorithm.

Table 3: Results of pSFS and the compared algorithms on the single diode model

\begin{tabular}{cccccccc}
\hline Algorithm & $I_{p h}(\mathrm{~A})$ & $I_{s d}(\mu \mathrm{A})$ & $R_{s h}(\Omega)$ & $R_{S}(\Omega)$ & $a$ & RMSE & Rank \\
\hline CLPSO & 0.76064 & 0.33454 & 56.03420 & 0.03623 & 1.48469 & $9.9207 \mathrm{E}-04$ & 7 \\
BLPSO & 0.76063 & 0.42518 & 62.58528 & 0.03523 & 1.50940 & $1.1239 \mathrm{E}-03$ & 8 \\
ABC & 0.76085 & 0.33016 & 53.59884 & 0.03629 & 1.48339 & $9.8815 \mathrm{E}-04$ & 6 \\
GOTLBO & 0.76077 & 0.32256 & 53.33877 & 0.03637 & 1.48106 & $9.8658 \mathrm{E}-04$ & 5 \\
TLABC & 0.76078 & 0.32302 & 53.71636 & 0.03638 & 1.48118 & $\mathbf{9 . 8 6 0 2 E - 0 4}$ & 1 \\
IJAYA & 0.76078 & 0.32304 & 53.71441 & 0.03638 & 1.48119 & $9.8603 \mathrm{E}-04$ & 4 \\
SFS & 0.76078 & 0.32302 & 53.71852 & 0.03638 & 1.48118 & $\mathbf{9 . 8 6 0 2 E - 0 4}$ & 1 \\
pSFS & 0.76078 & 0.32302 & 53.71852 & 0.03638 & 1.48118 & $\mathbf{9 . 8 6 0 2 E - 0 4}$ & 1 \\
\hline
\end{tabular}

The best results are highlighted in bold font. 


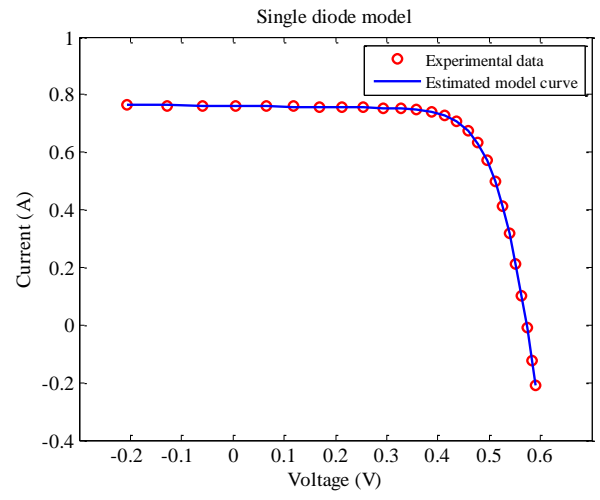

(a)

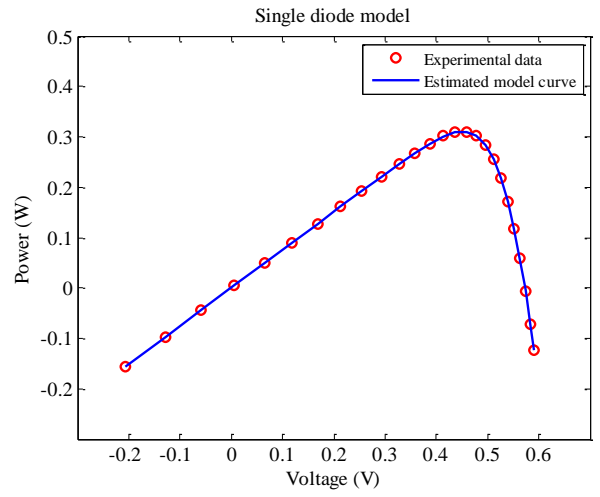

(b)

Figure 6: Comparisons between experimental data and the data estimated from pSFS for the single diode model: (a) $I$ - $V$ characteristics, (b) $P-V$ characteristics

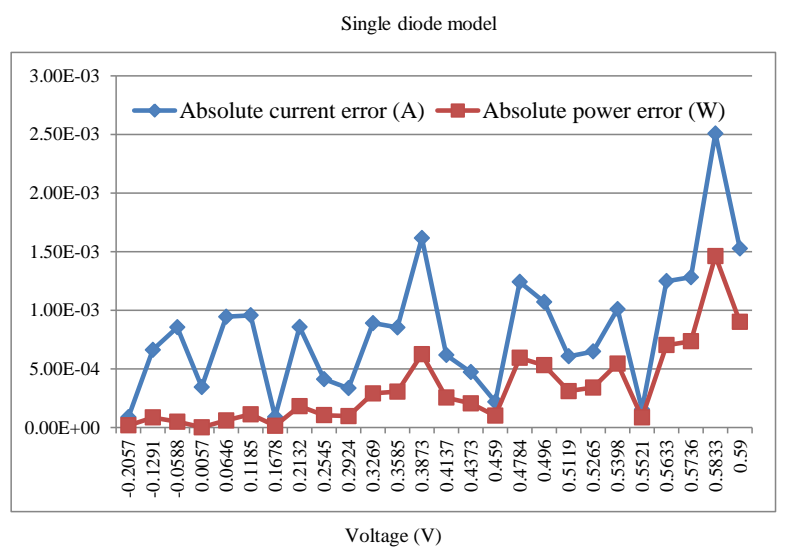

Figure 7: Individual absolute errors for current and power using pSFS (single diode model)

\subsubsection{Results on the double diode mode}

The comparison results of pSFS with the other seven algorithms for the DDM are presented in Table 4. Since there are seven unknown parameters for a DDM model, the estimation of the DDM is more complicated than that of the SDM.

From Table 4, it can be found that the proposed pSFS and the basic SFS attain the best RMSE value (i.e., 9.8255E-04). This indcates the higher accuracy of SFS algorithms over those non-SFS algorithms for PV parameter estimation. TLABC achieves the third best RMSE value (i.e., 9.8414E-04), and IJAVA obtains the fourth best RMSE value (i.e., 9.8423E-04). CLPSO and BLPSO exihit relative poor performance for this case, and their RMSE values are the worst among the eight algorithms.

To further evaluate the pSFS's estimation accuracy over the whole voltage range, Fig. 8 plots the $I$ - $V$ and $P-V$ characteristic curves. The estimated model curves of pSFS are in good agreement with the experimental data. Meanwhile, from Fig.9, the maximal IACE value is less than 2.6E-3, and the maximal IAPE value is less than 1.5E-3. These results show that the proposed pSFS also achieves a very high estimation accuracy for the DDM. 


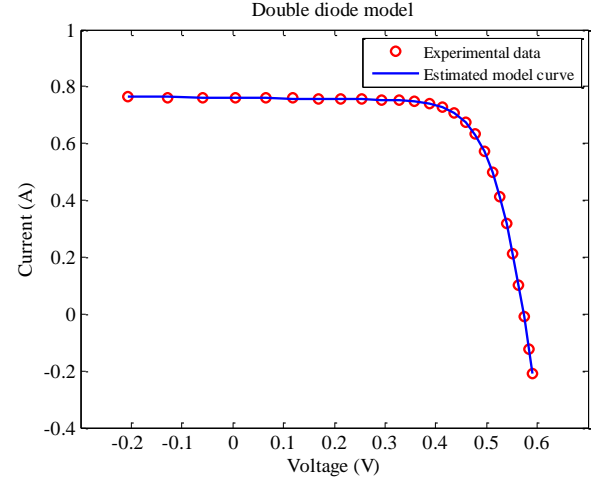

(a)

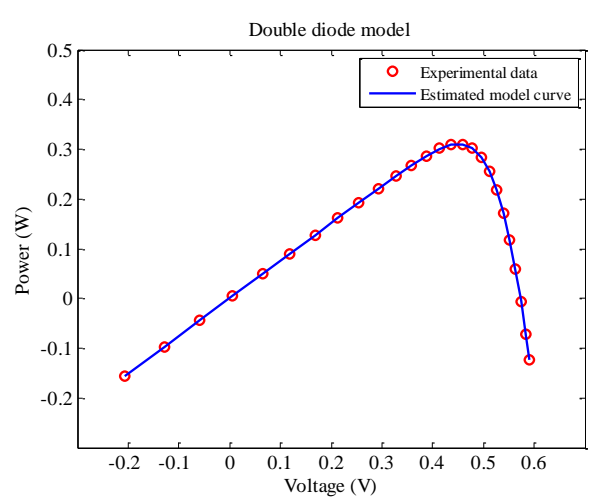

(b)

Figure 8: Comparisons between experimental data and the data estimated from pSFS for the double diode model: (a) $I$ - $V$ characteristics, (b) $P-V$ characteristics

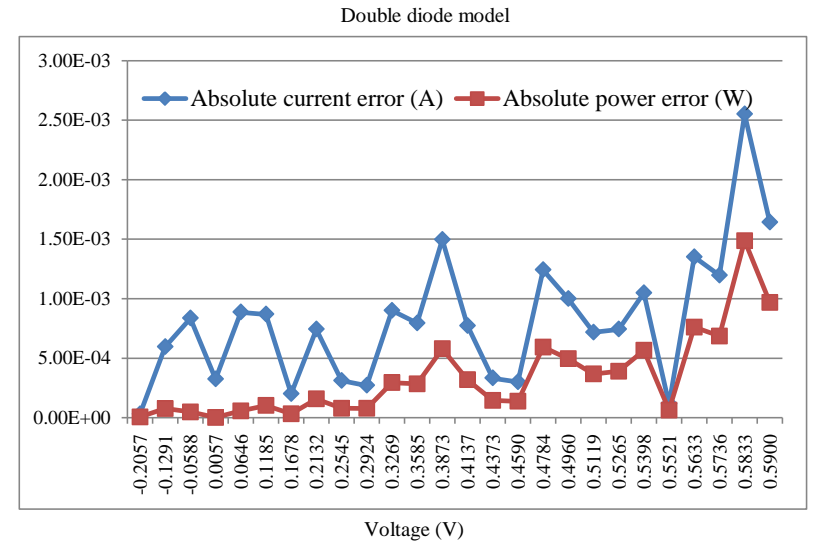

Figure 9: Individual absolute errors for current and power using pSFS (double diode model)

\subsubsection{Results on the PV module mode}

A PV module model that has five estimation parameters is taken to further evaluate the estimation accuracy of the proposed pSFS. Table 5 presents the comparison results of pSFS and the other algorithms. From the results in Table 5, three algorithms (i.e., pSFS, SFS and TLABC) achieve the best RMSE value (i.e., 2.42507E03), followed by IJAVA(2.42512E-03), GOTLBO(2.42513E-03), BLPSO (2.42520E-03), CLPSO (2.42661E-03) and $\mathrm{ABC}(2.44692 \mathrm{E}-03)$.

The optimal model parameters extracted by pSFS are used to construct the $I-V$ and $P-V$ curves. As 
shown in Fig 10, high consistency can be observed between the estimated model curves and the experimental data for both $I-V$ and $P-V$ characteristics. From Fig.11, it can be observed that the maximal IACE value is less than 8.0E-2, and the maximal IAPE value is less than 5.0E-3. All these comparisons demonstrate the high estimation accuracy of the proposed pSFS for the PV module model.

Table 5: Results of pSFS and the compared algorithms on the PV module model

\begin{tabular}{|c|c|c|c|c|c|c|c|}
\hline Algorithm & $I_{p h}(\mathrm{~A})$ & $I_{s d}(\mu \mathrm{A})$ & $R_{s h}(\Omega)$ & $R_{S}(\Omega)$ & $a$ & RMSE & Rank \\
\hline CLPSO & 1.03036 & 3.45208 & 1001.18353 & 1.20269 & 48.60837 & $2.42661 \mathrm{E}-03$ & 7 \\
\hline BLPSO & 1.03052 & 3.50258 & 983.15030 & 1.20062 & 48.66526 & $2.42520 \mathrm{E}-03$ & 6 \\
\hline $\mathrm{ABC}$ & 1.03008 & 3.30190 & 968.65121 & 1.20631 & 48.44085 & $2.44692 \mathrm{E}-03$ & 8 \\
\hline GOTLBO & 1.03046 & 3.49907 & 989.68885 & 1.20080 & 48.66113 & $2.42513 \mathrm{E}-03$ & 5 \\
\hline TLABC & 1.03052 & 3.48226 & 981.84265 & 1.20127 & 48.64284 & $2.42507 \mathrm{E}-03$ & 1 \\
\hline IJAYA & 1.03054 & 3.47922 & 981.13257 & 1.20145 & 48.63942 & $2.42512 \mathrm{E}-03$ & 4 \\
\hline SFS & 1.03051 & 3.48226 & 981.98237 & 1.20127 & 48.64283 & $2.42507 \mathrm{E}-03$ & 1 \\
\hline pSFS & 1.03051 & 3.48226 & 981.98223 & 1.20127 & 48.64283 & $2.42507 \mathrm{E}-03$ & 1 \\
\hline
\end{tabular}

The best results are highlighted in bold font.

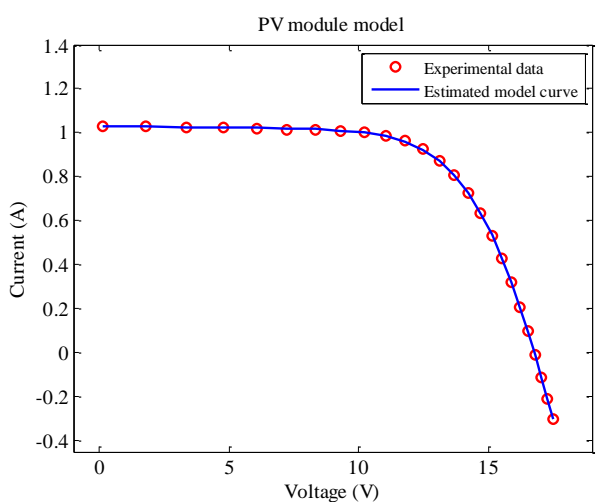

(a)

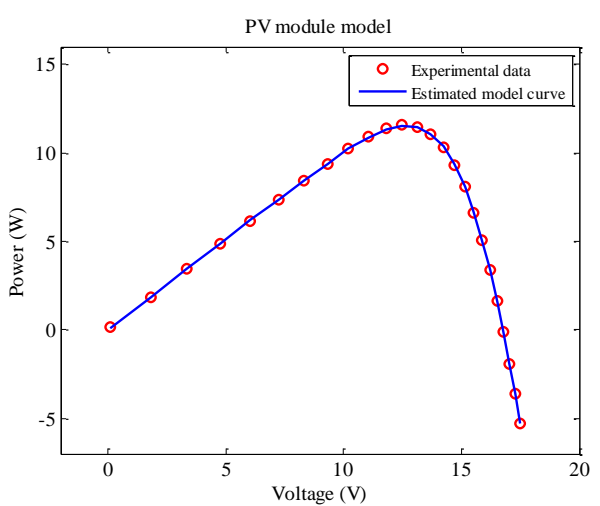

(b)

Figure 10: Comparisons between experimental data and the data estimated from pSFS for the PV module model: (a) $I$ - $V$ characteristics, (b) $P-V$ characteristics

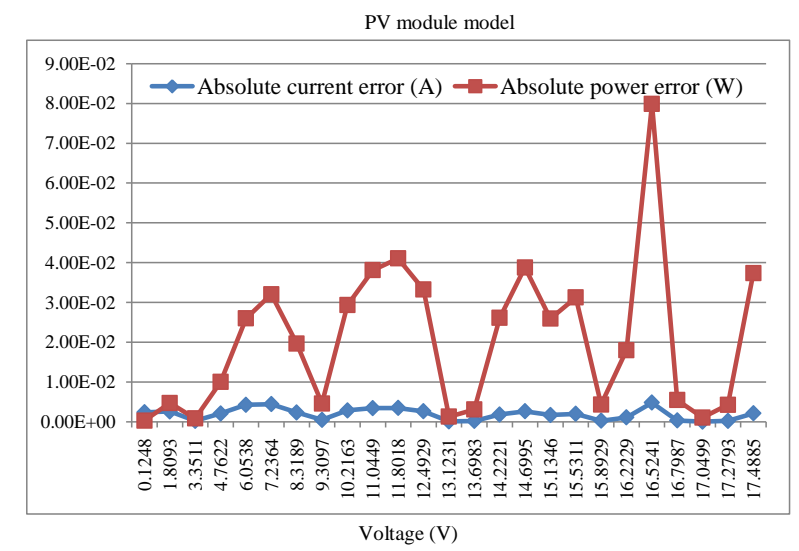

Figure 11: Individual absolute errors for current and power using pSFS (PV module model)

\subsection{Comparisons based on the statistical results}

Since all the eight PV parameter estimation algorithms are stochastic approaches, it is helpful to compare their statistical results. Table 6 presents the best, the mean, the worst and the standard deviation (SD) 
of RMSE of the eight algorithms over 30 independent runs. Again three PV models are considered in the comparison. In addition, the Wilcoxon rank sum test is conducted to compare pSFS with the other algorithms, so as to establish a statistical view. The symbols "+" and "=" indicate that pSFS performs significantly better or similar to others, with a significance level of $p=0.05$. The average computational times are also given in Table 6.

From Table 6, it can be found that:

- In terms of the best RMSE value, two SFS algorithms, i.e., pSFS and SFS, achieve the best results for all three PV models. TLABC achieves the best results for the SDM and PV module models. The other five algorithms, including CLPSO, BLPSO, ABC, GOTLBO and IJAVA, cannot obtain the best RMSE value on any $\mathrm{PV}$ models.

- Considering the mean and worst RMSE values, the proposed pSFS achieves the best results on two PV models, i.e., SDM and PV module. SFS gets the best result on the PV module model, but it performs worse than pSFS on SDM and DDM. This indicates that the chaotic elitist perturbation strategy improves the estimation accuracy of SFS. IJAVA is another competitive algorithm, as it attains the best result on DDM.

- The SD of RMSE can reflect the robustness of an algorithm in different runs. For this performance index, the proposed pSFS achieves the best results on the SDM and the PV module models. IJAVA gets the best SD result on DDM. It can also be observed that, pSFS achieves smaller SD values than SFS on all three PV models, which means the robustness of pSFS is enhanced by using the chaotic elitist perturbation strategy.

- According to the Wilcoxon rank sum test, the proposed pSFS performs significantly better than CLPSO, BLPSO, ABC, GOTLBO and TLABC on all three PV models. Compared with IJAVA and SFS, pSFS exhibits significantly better performance on the SDM and PV module.

- In terms of computational time, the eight algorithms use about 20 seconds for all three different PV models. Therefore, there are no clear differences among these algorithms.

Table 7 presents the Friedman rank values of the eight algorithms on the three problems PV parameter estimation problems. The proposed pSFS attains the best rank value (1.50), SFS the second(2.17), followed by IJAVA(2.67), TLABC(4.33), CLPSO(5.67), GOTLBO(6.33), ABC(6.33) and BLPSO(7.00).

Based on the above statistical comparisons and analysis, it can be concluded that the proposed pSFS achieves the overall best parameter estimation accuracy and robustness among the eight algorithms. The chaotic elitist perturbation strategy enhances the performance of the pSFS algorithm. Therefore, pSFS can be used as an accurate and robust method for PV parameter estimation problems with different diode models. 
Table 6: Statistical results of pSFS and the compared algorithms for different PV models

Table 7: Friedman rank values of theses algorithms on all three problems

\begin{tabular}{lcc}
\hline & Friedman rank & Final rank \\
\hline CLPSO & 5.67 & 5 \\
BLPSO & 7.00 & 8 \\
ABC & 6.33 & 6 \\
GOTLBO & 6.33 & 7 \\
TLABC & 4.33 & 4 \\
IJAYA & 2.67 & 3 \\
SFS & 2.17 & 2 \\
pSFS & 1.50 & 1 \\
\hline
\end{tabular}

\subsection{Comparisons of the convergence}

We also compare the convergence of the eight algorithms for the three PV models. Fig.12 plots the convergence curves in terms of average RMSE. From Fig.12, it can be observed that:

- IJAVA has the fastest convergence speed, followed by pSFS, SFS, TLABC and GOTLBO.

- The proposed pSFS has reasonably fast convergence speed. Meanwhile, it achieves high final estimation accuracy for all three PV models.

- pSFS converges faster than SFS for all three PV models. This indicates the chaotic elitist perturbation strategy accelerates its convergence speed.

- GOTLBO and TLABC have the similar convergence speed as that of pSFS, but their final estimation accuracy is worse than pSFS.

- The other three algorithms, BLPSO, CLPSO and ABC, converge relative slow. Also, their estimation accuracy are lower in comparison with other algorithms. 
Based on the above comparisions, it can be concluded that the proposed pSFS has a reasonably fast convergence speed, and its final convergence accuracy is the highest compared with all the other algorithms. In fact, an efficient seach process should balance the exploration and exploitation. In other words, too fast converge speed may make algorithms premature, while too solw converge speed may affect the final solution accuracy. For the multimodal PV parameter estimation problem, the proposed pSFS can keep an effective balance between global exploration and local exploitation. This is probably why pSFS can achieve high final estimation accuracy for all three PV models.

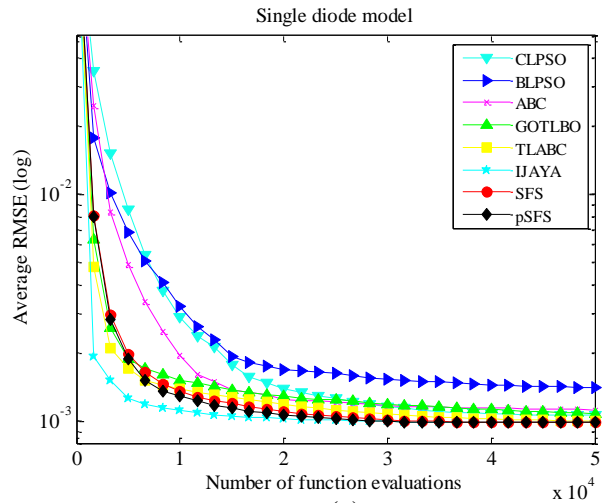

(a)

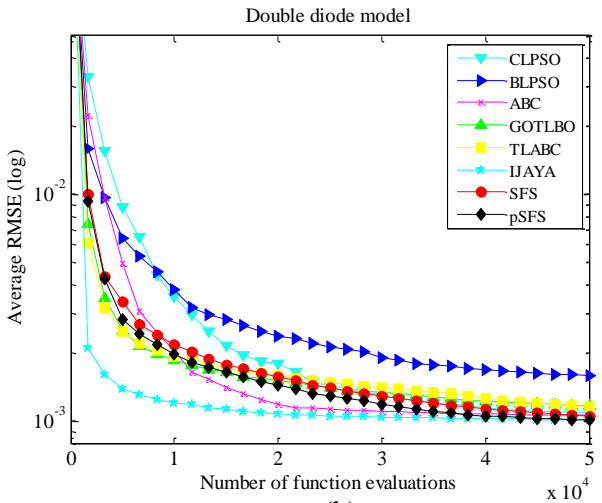

(b)

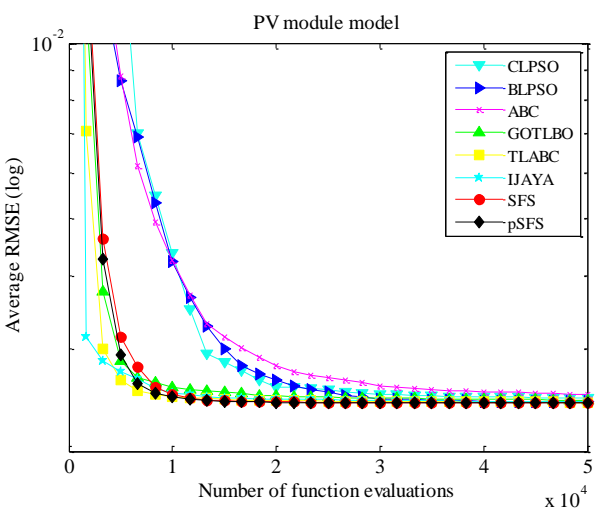

(c)

Figure 12: Convergence graphs of different algorithms for three PV models (a) SDM (b) DDM (c) PV module

\subsection{Parameter analysis}

To implement an efficient algorithm, it is also very important to analyze the tuning parameters. We analyze the impacts of the population size and the two Gaussian random walks on the performance of the proposed pSFS algorithm.

\subsubsection{Population size}

Table 8 shows the results of the proposed pSFS algorithm with population size $N P=10,20,30,40$ and 50 for three PV estimation problems. From the results listed in Table 8, it can be observed that:

- For the SDM, pSFS-NP30 yields the best performance for all four statistical results. Although pSFSNP20, pSFS-NP40, and pSFS-NP50 also provides the best results in terms of best RMSE, their results 
are not as good as pSFS-NP30 when comparing other performance indices.

- For the DDM, pSFS-NP30 obtains the best results in terms of worst and SD of RMSE.

- For the PV module model, pSFS-NP30 yields the best performance in terms of best, mean, worst and SD of RMSE. Meanwhile, pSFS-NP30 is the only algorithm which gets the minimal SD value.

These results indicate that pSFS with a moderate population size (i.e., pSFS-NP30) has the overall highest estimation accuracy and robustness. In fact, when the population size is too small, the population diversity is poor, which affects the global exploration and estimation accuracy. On the contrary, when the population size is too large, the algorithm needs more computational overheads (i.e., number of function evaluations) to converge. When the maximum number of function evaluations is fixed, too large population size may deteriorate the final performance. For the PV estimation problems, a moderate population size $N P=30$ is recommended for pSFS.

Table 8: Comparison of the pSFS algorithm with different population size for three PV models

\begin{tabular}{|c|c|c|c|c|c|}
\hline & & $\begin{array}{l}\text { RMSE } \\
\text { Min }\end{array}$ & Mean & $\operatorname{Max}$ & SD \\
\hline \multirow[t]{5}{*}{$\overline{\mathrm{SDM}}$} & pSFS-N10 & $9.8851 \mathrm{E}-04$ & $1.6630 \mathrm{E}-03$ & $3.6544 \mathrm{E}-03$ & $6.1567 \mathrm{E}-04$ \\
\hline & pSFS-N20 & $9.8602 \mathrm{E}-04$ & $1.0072 \mathrm{E}-03$ & $1.0854 \mathrm{E}-03$ & $3.2138 \mathrm{E}-05$ \\
\hline & pSFS-N30 & $9.8602 \mathrm{E}-04$ & $9.8602 \mathrm{E}-04$ & $9.8608 \mathrm{E}-04$ & $1.1063 \mathrm{E}-08$ \\
\hline & pSFS-N40 & 9.8602E-04 & $9.8603 \mathrm{E}-04$ & $9.8628 \mathrm{E}-04$ & $4.7319 \mathrm{E}-08$ \\
\hline & pSFS-N50 & $9.8602 \mathrm{E}-04$ & $9.8666 \mathrm{E}-04$ & $9.9449 \mathrm{E}-04$ & $1.6666 \mathrm{E}-06$ \\
\hline \multirow[t]{5}{*}{ DDM } & pSFS-N10 & $9.8774 \mathrm{E}-04$ & $1.8195 \mathrm{E}-03$ & $5.5085 \mathrm{E}-03$ & $1.0045 \mathrm{E}-03$ \\
\hline & pSFS-N20 & $9.8248 \mathrm{E}-04$ & $1.0946 \mathrm{E}-03$ & $2.2346 \mathrm{E}-03$ & $2.3917 \mathrm{E}-04$ \\
\hline & pSFS-N30 & $9.8255 \mathrm{E}-04$ & $1.0122 \mathrm{E}-03$ & 1.1930E-03 & 4.6733E-05 \\
\hline & pSFS-N40 & $9.8268 \mathrm{E}-04$ & $1.0118 \mathrm{E}-03$ & $1.3399 \mathrm{E}-03$ & $7.5648 \mathrm{E}-05$ \\
\hline & pSFS-N50 & $9.8291 \mathrm{E}-04$ & $1.0411 \mathrm{E}-03$ & $1.4825 \mathrm{E}-03$ & $1.0548 \mathrm{E}-04$ \\
\hline \multirow[t]{5}{*}{ PV module } & pSFS-N10 & $2.42570 \mathrm{E}-03$ & $1.22912 \mathrm{E}-02$ & $2.74251 \mathrm{E}-01$ & $4.94925 \mathrm{E}-02$ \\
\hline & pSFS-N20 & $2.42507 \mathrm{E}-03$ & $2.46508 \mathrm{E}-03$ & $3.17858 \mathrm{E}-03$ & $1.51147 \mathrm{E}-04$ \\
\hline & pSFS-N30 & 2.42507 E-03 & 2.42507 E-03 & 2.42507E-03 & $6.62209 \mathrm{E}-17$ \\
\hline & pSFS-N40 & $2.42507 \mathrm{E}-03$ & $2.42507 \mathrm{E}-03$ & $2.42508 \mathrm{E}-03$ & $2.53130 \mathrm{E}-10$ \\
\hline & pSFS-N50 & $2.42507 \mathrm{E}-03$ & $2.42507 \mathrm{E}-03$ & $2.42508 \mathrm{E}-03$ & $3.13602 \mathrm{E}-10$ \\
\hline
\end{tabular}

\subsubsection{Gaussian random walks}

Table 9 shows the results of the pSFS with two different Gaussian walks (GW). From Table 9, it can be found that:

- For the SDM, both pSFS-GW1 and pSFS-GW2 achieve the best results for the best RMSE. However, pSFS-GW1 yields better performance than pSFS-GW2 when considering the mean, worst and SD of RMSE.

- For the DDM, pSFS-GW2 achieves the better results for the best RMSE. pSFS-GW1 yields better performance than pSFS-GW2 when considering the mean, worst and SD of RMSE.

- For the PV module model, both pSFS-GW1 and pSFS-GW2 get the best results in terms of best, mean and worst RMSE. pSFS-GW2 obtains a smaller SD value than pSFS-GW1.

Our results indicate that there is no significant difference between pSFS-GW1 and pSFS-GW2 for the PV estimation problems. According to reports [28, pSFS-GW1 has a faster convergence speed and is more suitable for solving simple problems. pSFS-GW2 is more suitable for complicated problems, which needs more 
iteration number to converge. From the case study results in this work, pSFS-GW1 is recommended for the PV estimation problems.

Table 9: Comparison of the pSFS algorithm with two kinds of Gaussian walks

\begin{tabular}{|c|c|c|c|c|c|}
\hline & & $\begin{array}{c}\text { RMSE } \\
\text { Best }\end{array}$ & Mean & Worst & SD \\
\hline \multirow[t]{2}{*}{ SDM } & pSFS-GW1 & $9.8602 \mathrm{E}-04$ & $9.8602 \mathrm{E}-04$ & $9.8608 \mathrm{E}-04$ & $1.1063 \mathrm{E}-08$ \\
\hline & pSFS-GW2 & $9.8602 \mathrm{E}-04$ & $9.8608 \mathrm{E}-04$ & $9.8782 \mathrm{E}-04$ & $3.2842 \mathrm{E}-07$ \\
\hline \multirow[t]{2}{*}{ DDM } & pSFS-GW1 & $9.8255 \mathrm{E}-04$ & 1.0122E-03 & 1.1930E-03 & $4.6733 \mathrm{E}-05$ \\
\hline & pSFS-GW2 & $9.8249 \mathrm{E}-04$ & $1.0113 \mathrm{E}-03$ & $1.4070 \mathrm{E}-03$ & $8.3206 \mathrm{E}-05$ \\
\hline \multirow[t]{2}{*}{ PV module } & pSFS-GW1 & 2.42507 E-03 & $2.42507 \mathrm{E}-03$ & $2.42507 \mathrm{E}-03$ & $6.62209 \mathrm{E}-17$ \\
\hline & pSFS-GW2 & $2.42507 \mathrm{E}-03$ & 2.42507 E-03 & 2.42507 E-03 & $4.98125 \mathrm{E}-17$ \\
\hline
\end{tabular}

\section{Validation of pSFS with survey experimental data}

The above comparison studies demonstrate that pSFS has the highest estimation accuracy and robustness for parameter estimation for PV models. In this section, the practical use of pSFS is further examined by using experimental data from three different PV modules in the manufacturer data sheet: Thin-film ST40, Mono-crystalline SM55 and Multi-crystalline KC200GT [14].

The experimental $I-V$ data are extracted directly from the data sheet of five different irradiation levels at different temperature levels. The searching ranges for the five unknown parameters are: $I_{p h} \in\left[0,2 I_{s c}\right](\mathrm{A})$, $I_{s d} \in[0,100](\mu \mathrm{A}), R_{s} \in[0,2](\Omega), R_{s h} \in[0,5000](\Omega)$ and $a \in[1,4]$. The short circuit current $I_{s c}$ at nonstandard condition is calculated by Eq.(17):

$$
I_{s c}(G, T)=I_{s c \_S T C} \frac{G}{G_{S T C}}+\alpha\left(T-T_{S T C}\right)
$$

where $G$ and $T$ are the irradiation and the temperature levels, respectively; $G_{S T C}=1000 \mathrm{~W} / \mathrm{m}^{2}$ and $T_{S T C}=$ $25^{\circ} \mathrm{C} ; I_{\text {Sc_STC }}$ is short circuit current at standard test condition .

Table 10 and Table 11 present the optimal model parameters extracted by the proposed pSFS algorithm for three PV models under different irradiation and temperature levels, respectively. Furthermore, to verify the accuracy of the model parameters, the $I-V$ characteristics of the three different PV modules under different irradiation and temperature levels are plotted in Fig. 13 and Fig. 14, respectively.

From the results in Table 10 and Table 11, the optimal model parameters extracted by the proposed pSFS are in close with those by MPSO [14, and low RMSE values are achieved at different irradiation and temperature levels. From Fig. 13 and Fig. 14, the model curves calculated from the extracted model parameters match well with the experimental data under various environment conditions, i.e., at different irradiance and temperature levels. These observations indicate that the proposed pSFS approach can accurately extract the model parameters when the PV systems are under certain mismatch conditions such as partial shading. 
Table 10: Model parameters estimated by the pSFS algorithm for three PV modules at different irradiance and temperature of $25^{\circ} \mathrm{C}$

\begin{tabular}{|c|c|c|c|}
\hline Parameters & Thin-film ST40 & Mono-crystalline SM55 & Multi-crystalline $\mathrm{KC} 200 \mathrm{GT}$ \\
\hline \multicolumn{4}{|c|}{$G=1000 \mathrm{~W} / \mathrm{m}^{2}$} \\
\hline$I_{p h}(\mathrm{~A})$ & 2.67580 & 3.45010 & 8.21688 \\
\hline$I_{s d}(\mu \mathrm{A})$ & 1.52880 & 0.17115 & 0.00225 \\
\hline$R_{S}(\Omega)$ & 1.11323 & 0.32915 & 0.34376 \\
\hline$R_{s h}(\Omega)$ & 357.59844 & 483.90046 & 763.51258 \\
\hline$a$ & 1.50028 & 1.39575 & 1.07653 \\
\hline RMSE & $7.34099 \mathrm{E}-04$ & $1.14621 \mathrm{E}-03$ & $1.53933 \mathrm{E}-03$ \\
\hline \multicolumn{4}{|c|}{$G=800 \mathrm{~W} / \mathrm{m}^{2}$} \\
\hline$I_{p h}(\mathrm{~A})$ & 2.13801 & 2.76038 & 6.57104 \\
\hline$I_{s d}(\mu \mathrm{A})$ & 1.15810 & 0.14395 & 0.00098 \\
\hline$R_{S}(\Omega)$ & 1.12529 & 0.33759 & 0.35678 \\
\hline$R_{s h}(\Omega)$ & 332.88893 & 459.87849 & 754.80161 \\
\hline$a$ & 1.47315 & 1.38114 & 1.03680 \\
\hline RMSE & $7.73905 \mathrm{E}-04$ & $6.68579 \mathrm{E}-04$ & $1.64367 \mathrm{E}-03$ \\
\hline \multicolumn{4}{|c|}{$G=600 \mathrm{~W} / \mathrm{m}^{2}$} \\
\hline$I_{p h}(\mathrm{~A})$ & 1.60481 & 2.07090 & 4.93431 \\
\hline$I_{s d}(\mu \mathrm{A})$ & 1.44187 & 0.15551 & 0.00386 \\
\hline$R_{S}(\Omega)$ & 1.11261 & 0.33050 & 0.33734 \\
\hline$R_{s h}(\Omega)$ & 347.69469 & 450.06853 & 743.00159 \\
\hline$a$ & 1.49582 & 1.38753 & 1.10402 \\
\hline RMSE & $6.74036 \mathrm{E}-04$ & $8.23949 \mathrm{E}-04$ & $1.29767 \mathrm{E}-03$ \\
\hline \multicolumn{4}{|c|}{$G=400 \mathrm{~W} / \mathrm{m}^{2}$} \\
\hline$I_{p h}(\mathrm{~A})$ & 1.06754 & 1.38284 & 3.28785 \\
\hline$I_{s d}(\mu \mathrm{A})$ & 1.84875 & 0.10042 & 0.00149 \\
\hline$R_{S}(\Omega)$ & 1.08058 & 0.39665 & 0.35358 \\
\hline$R_{s h}(\Omega)$ & 362.51450 & 427.05044 & 752.08941 \\
\hline$a$ & 1.52445 & 1.35199 & 1.05504 \\
\hline RMSE & $6.30725 \mathrm{E}-04$ & $7.07608 \mathrm{E}-04$ & $1.42620 \mathrm{E}-03$ \\
\hline \multicolumn{4}{|c|}{$G=200 \mathrm{~W} / \mathrm{m}^{2}$} \\
\hline$I_{p h}(\mathrm{~A})$ & 0.53314 & 0.69151 & 1.64615 \\
\hline$I_{s d}(\mu \mathrm{A})$ & 1.42968 & 0.14641 & 0.00052 \\
\hline$R_{S}(\Omega)$ & 1.18572 & 0.28662 & 0.38111 \\
\hline$R_{s h}(\Omega)$ & 344.98324 & 448.21071 & 690.14660 \\
\hline$a$ & 1.49752 & 1.38066 & 1.00324 \\
\hline RMSE & $4.77201 \mathrm{E}-04$ & $3.20688 \mathrm{E}-04$ & $1.41847 \mathrm{E}-03$ \\
\hline
\end{tabular}

Table 11: Model parameters estimated by pSFS for three PV modules at different temperature and irradiance of $1000 \mathrm{~W} / \mathrm{m}^{2}$

\begin{tabular}{|c|c|c|c|c|c|c|c|}
\hline & Temperature & $I_{p h}(\mathrm{~A})$ & $I_{s d}(\mu \mathrm{A})$ & $R_{S}(\Omega)$ & $R_{s h}(\Omega)$ & $a$ & RMSE \\
\hline \multirow{4}{*}{ Thin-film ST40 } & $25^{\circ} \mathrm{C}$ & 2.67580 & 1.52880 & 1.11323 & 357.59844 & 1.50028 & 7.34099E-04 \\
\hline & $40^{\circ} \mathrm{C}$ & 2.68091 & 5.66610 & 1.12930 & 364.10973 & 1.47648 & $1.32141 \mathrm{E}-03$ \\
\hline & $55^{\circ} \mathrm{C}$ & 2.69197 & 18.68073 & 1.14959 & 295.02177 & 1.44978 & $1.82326 \mathrm{E}-03$ \\
\hline & $70^{\circ} \mathrm{C}$ & 2.69233 & 87.52185 & 1.12589 & 367.75322 & 1.54824 & $7.77718 \mathrm{E}-04$ \\
\hline \multirow{3}{*}{ Mono-crystalline SM55 } & $25^{\circ} \mathrm{C}$ & 3.45010 & 0.17115 & 0.32915 & 483.90046 & 1.39575 & $1.14621 \mathrm{E}-03$ \\
\hline & $40^{\circ} \mathrm{C}$ & 3.46914 & 1.14511 & 0.31310 & 533.06920 & 1.41784 & $3.78881 \mathrm{E}-03$ \\
\hline & $60^{\circ} \mathrm{C}$ & 3.49461 & 6.90950 & 0.31871 & 484.88387 & 1.40514 & $3.78039 \mathrm{E}-03$ \\
\hline \multirow{3}{*}{ Multi-crystalline KC200GT } & $25^{\circ} \mathrm{C}$ & 8.21688 & 0.00225 & 0.34376 & 763.51258 & 1.07653 & $1.53933 \mathrm{E}-03$ \\
\hline & $50^{\circ} \mathrm{C}$ & 8.29531 & 0.12595 & 0.33565 & 953.88989 & 1.11729 & $2.74651 \mathrm{E}-03$ \\
\hline & $75^{\circ} \mathrm{C}$ & 8.37766 & 1.63082 & 0.34250 & 790.55822 & 1.10148 & 4.47293E-03 \\
\hline
\end{tabular}




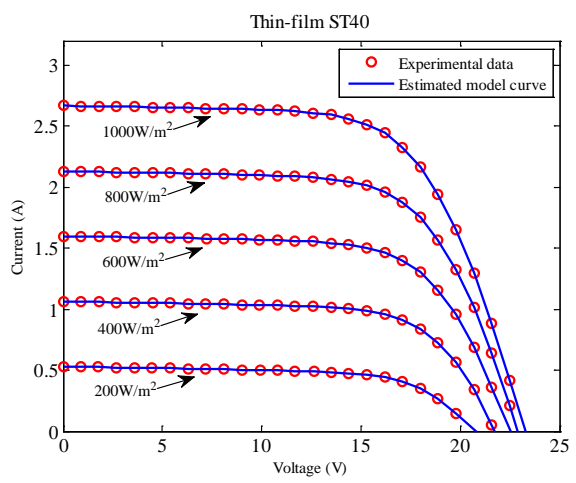

(a)

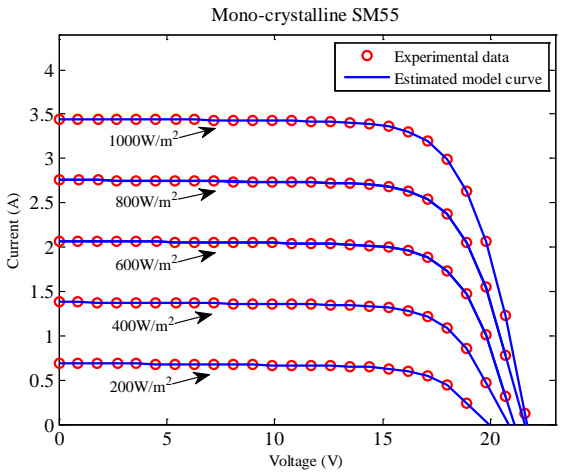

(b)

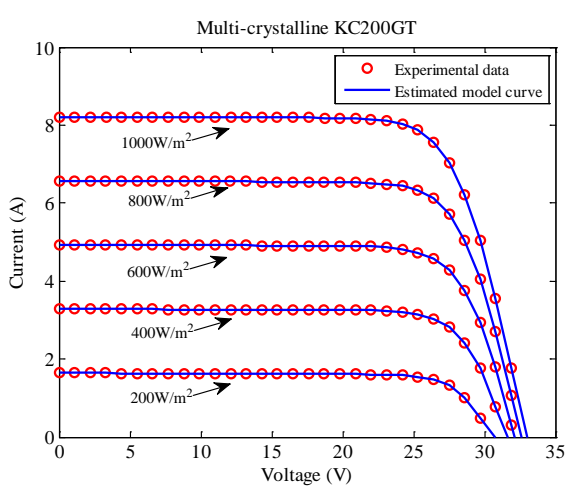

(c)

Figure 13: Comparisons between the experimental data and estimated data obtained by pSFS for three PV models at different irradiance, using real data from: (a) Thin-film ST40; (b) Mon-crystalline SM55; (c) Multi-crystalline KC200GT. 


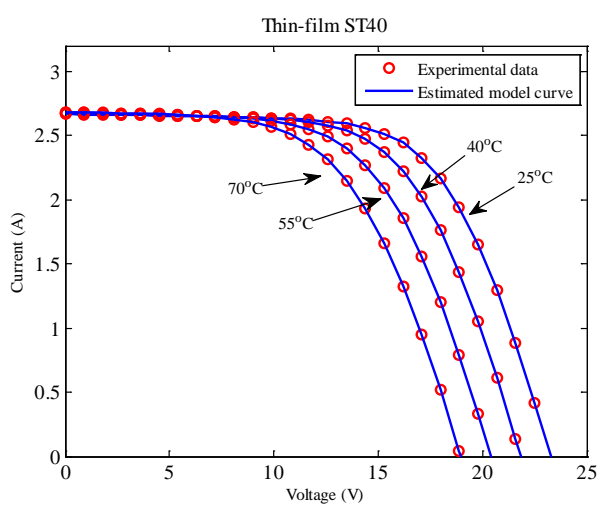

(a)

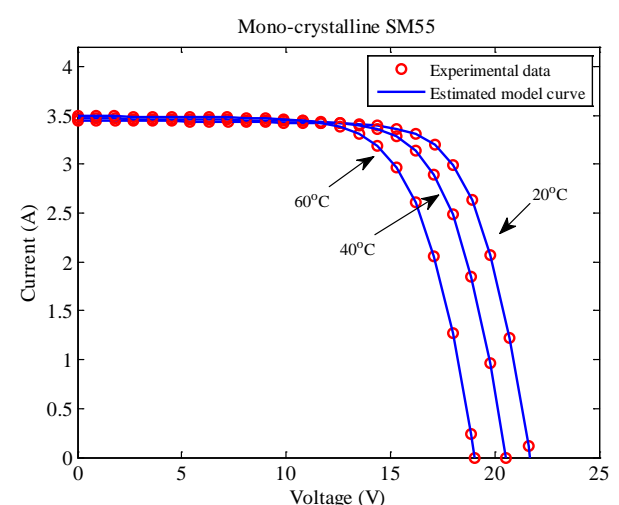

(b)

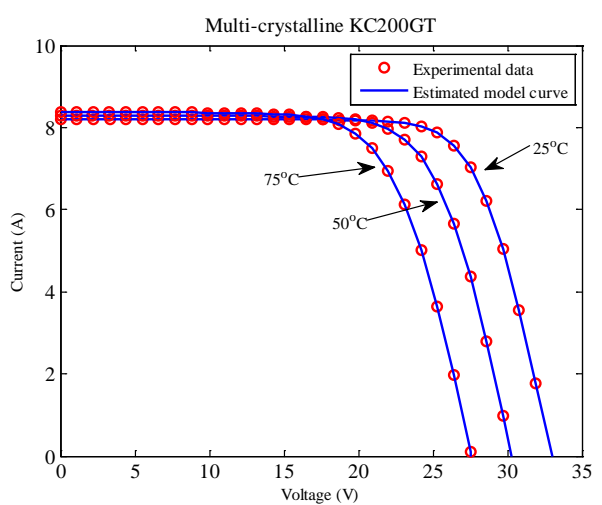

(c)

Figure 14: Comparisons between the experimental data and estimated data obtained by pSFS for three PV models at different temperatures, using real data from: (a) Thin-film ST40; (b) Mon-crystalline SM55; (c) Multi-crystalline KC200GT.

\section{Conclusion}

In this work, we have proposed a perturbed stochastic fractal search (pSFS) algorithm to accurately and robustly extract the PV model parameters. The proposed pSFS algorithm employs diffusion and updating processes inspired from random fractal properties. Meanwhile, a chaotic elitist perturbation strategy is also employed to perform self-adaptive local search around the best solution in each generation. The proposed pSFS algorithm is evaluated on PV parameter estimation problems with different diode models. Also, the performance of pSFS is compared with the basic SFS and six non-SFS algorithms. The following conclusions can be drawn from the numerical results.

- pSFS achieves high parameter estimation accuracy for different PV models. The statistical results demonstrate that pSFS has the best results in terms of the optimal, the mean and the worst RMSE values. Moreover, for the SDM and DDM, the absolute current error of pSFS is smaller than $0.3 \%$. For the PV module, the absolute current error of pSFS is smaller than $8 \%$.

- pSFS also has superiority in robustness compared with the recently developed algorithms including SFS, IJAVA, TLABC and GOTLBO. For the SDM, pSFS achieves the smallest SD value, the value of which 
is smaller than 1e-7. For the PV module, pSFS achieves the smallest SD value less than 1e-16.

- pSFS performs better than the other compared algorithms under comparison according to the Wilcoxon rank sum test.

- pSFS has a reasonably fast convergence speed, and keeps a good balance between global exploration and local exploitation during the searching process by employing two search operators.

- pSFS is robust to various environment conditions. The tests on three real PV modules at different irradiance and temperature levels show that pSFS achieves accurate results under all circumstances.

The proposed pSFS gains the above benefits mainly from two aspects. The first is the diffusion and updating processes from the basic SFS, which helps to achieve balance between global and local search. The second is the chaotic elitist perturbation strategy, which further enhances the estimation accuracy and robustness. In the future, we are interested in applying the pSFS algorithm to modeling of more complicated PV systems such as integral and fractional order dynamic PV system models. This novel algorithm can be applied to wider energy optimization problems, such as economic dispatch and unit commitment.

\section{Acknowledgement}

This work was supported by the UK Engineering and Physical Sciences Research Council (EPSRC) (Grant No. EP/R007497/1), the Natural Science Foundation of Jiangsu Province, China (Grant No. BK 20160540), and the National Natural Science Foundation of China (Grant No. 61806179).

\section{Appendix 1 Pseudocode of the pSFS algorithm}

\section{References}

[1] H. Chen, S. Jiao, A. A. Heidari, M. Wang, X. Chen, X. Zhao, An opposition-based sine cosine approach with local search for parameter estimation of photovoltaic models, Energy Conversion and Management 195 (2019) 927-942.

[2] S. Xu, Y. Wang, Parameter estimation of photovoltaic modules using a hybrid flower pollination algorithm, Energy Conversion and Management 144 (2017) 53-68.

[3] D. Alam, D. Yousri, M. Eteiba, Flower pollination algorithm based solar pv parameter estimation, Energy Conversion and Management 101 (2015) 410-422.

[4] K. Yu, J. Liang, B. Qu, Z. Cheng, H. Wang, Multiple learning backtracking search algorithm for estimating parameters of photovoltaic models, Applied Energy 226 (2018) 408-422.

[5] A. Ortiz-Conde, F. J. G. Sánchez, J. Muci, New method to extract the model parameters of solar cells from the explicit analytic solutions of their illuminated i-v characteristics, Solar Energy Materials and Solar Cells 90 (2006) 352-361. 


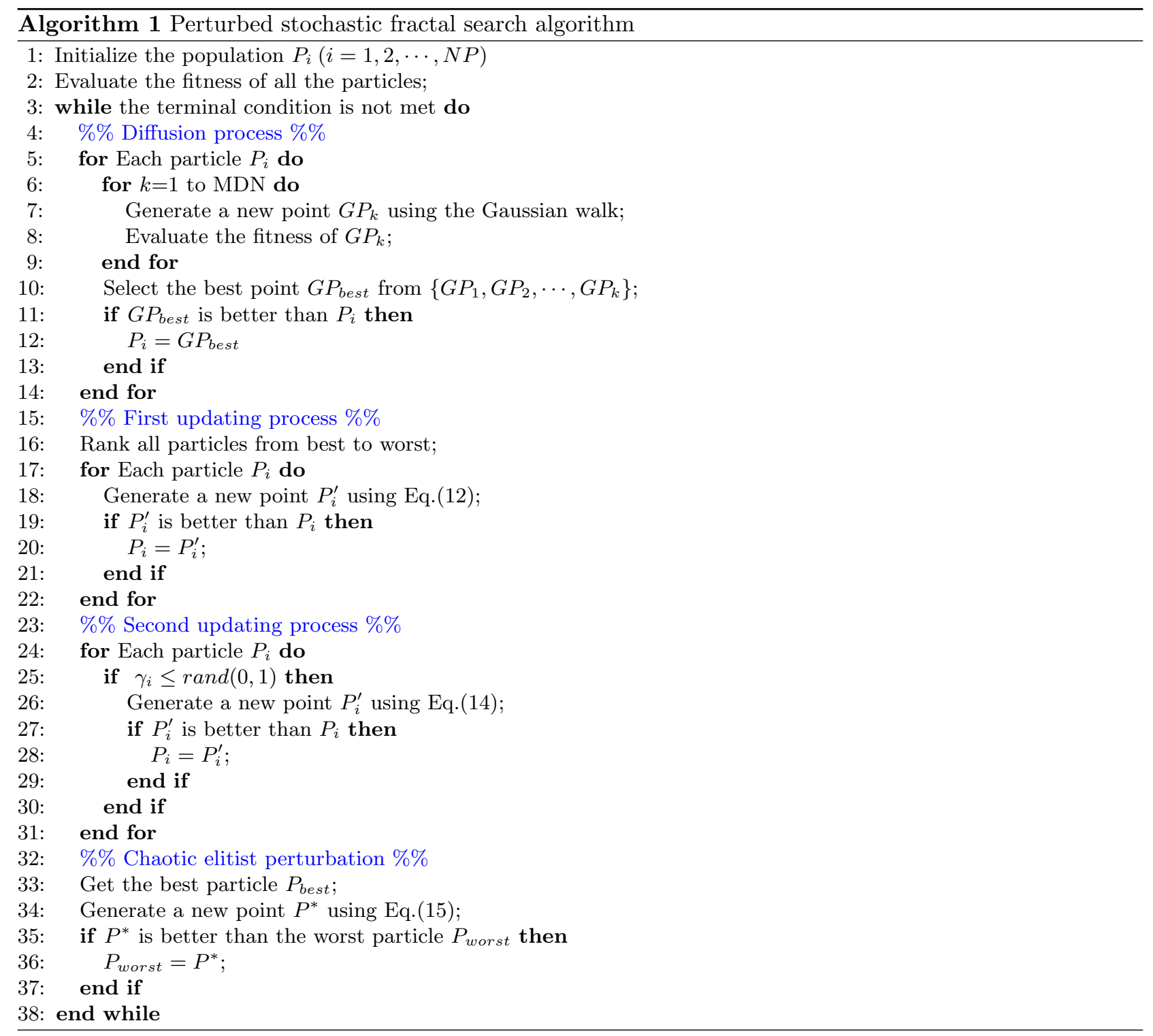

[6] T. Easwarakhanthan, J. Bottin, I. Bouhouch, C. Boutrit, Nonlinear minimization algorithm for determining the solar cell parameters with microcomputers, International Journal of Solar Energy 4 (1986) $1-12$.

[7] D. Chan, J. Phillips, J. Phang, A comparative study of extraction methods for solar cell model parameters, Solid-State Electronics 29 (1986) 329-337.

[8] X. Chen, H. Tianfield, , K. Li, Self-adaptive differential artificial bee colony algorithm for global optimization problems, Swarm and Evolutionary Computation 45 (2019) 70-91.

[9] B. Xu, X. Chen, L. Tao, Differential evolution with adaptive trial vector generation strategy and clusterreplacement-based feasibility rule for constrained optimization, Information Sciences 435 (2018) 240-262.

[10] Z. Zhu, L. Chen, C. Yuan, C. Xia, Global replacement-based differential evolution with neighbor-based memory for dynamic optimization, Applied Intelligence 48 (2018) 3280-3294. 
[11] A. A. Nagra, F. Han, Q.-H. Ling, S. Mehta, An improved hybrid method combining gravitational search algorithm with dynamic multi swarm particle swarm optimization, IEEE Access 7 (2019) 50388-50399.

[12] L. L. Jiang, D. L. Maskell, J. C. Patra, Parameter estimation of solar cells and modules using an improved adaptive differential evolution algorithm, Applied Energy 112 (2013) 185-193.

[13] A. R. Jordehi, Enhanced leader particle swarm optimisation (elpso): An efficient algorithm for parameter estimation of photovoltaic (pv) cells and modules, Solar Energy 159 (2018) 78-87.

[14] M. Merchaoui, A. Sakly, M. F. Mimouni, Particle swarm optimisation with adaptive mutation strategy for photovoltaic solar cell/module parameter extraction, Energy Conversion and Management 175 (2018) $151-163$.

[15] D. Yousri, D. Allam, M. Eteiba, P. N. Suganthan, Static and dynamic photovoltaic models parameters identification using chaotic heterogeneous comprehensive learning particle swarm optimizer variants, Energy Conversion and Management 182 (2019) 546-563.

[16] A. Askarzadeh, A. Rezazadeh, Parameter identification for solar cell models using harmony search-based algorithms, Solar Energy 86 (2012) 3241-3249.

[17] D. Oliva, E. Cuevas, G. Pajares, Parameter identification of solar cells using artificial bee colony optimization, Energy 72 (2014) 93-102.

[18] X. Chen, K. Yu, W. Du, W. Zhao, G. Liu, Parameters identification of solar cell models using generalized oppositional teaching learning based optimization, Energy 99 (2016) 170-180.

[19] D. Oliva, M. A. El Aziz, A. E. Hassanien, Parameter estimation of photovoltaic cells using an improved chaotic whale optimization algorithm, Applied Energy 200 (2017) 141-154.

[20] G. Xiong, J. Zhang, D. Shi, Y. He, Parameter extraction of solar photovoltaic models using an improved whale optimization algorithm, Energy Conversion and Management 174 (2018) 388-405.

[21] K. Yu, B. Qu, C. Yue, S. Ge, X. Chen, J. Liang, A performance-guided jaya algorithm for parameters identification of photovoltaic cell and module, Applied Energy 237 (2019) 241-257.

[22] R. Abbassi, A. Abbassi, A. A. Heidari, S. Mirjalili, An efficient salp swarm-inspired algorithm for parameters identification of photovoltaic cell models, Energy Conversion and Management 179 (2019) 362-372.

[23] T. S. Babu, J. P. Ram, K. Sangeetha, A. Laudani, N. Rajasekar, Parameter extraction of two diode solar pv model using fireworks algorithm, Solar Energy 140 (2016) 265-276.

[24] A. M. Beigi, A. Maroosi, Parameter identification for solar cells and module using a hybrid firefly and pattern search algorithms, Solar Energy 171 (2018) 435-446.

[25] X. Chen, B. Xu, C. Mei, Y. Ding, K. Li, Teaching-learning-based artificial bee colony for solar photovoltaic parameter estimation, Applied Energy 212 (2018) 1578-1588. 
[26] X. Chen, K. Yu, Hybridizing cuckoo search algorithm with biogeography-based optimization for estimating photovoltaic model parameters, Solar Energy 180 (2019) 192-206.

[27] L. Wu, Z. Chen, C. Long, S. Cheng, P. Lin, Y. Chen, H. Chen, Parameter extraction of photovoltaic models from measured iv characteristics curves using a hybrid trust-region reflective algorithm, Applied Energy 232 (2018) 36-53.

[28] H. Salimi, Stochastic fractal search: a powerful metaheuristic algorithm, Knowledge-Based Systems 75 (2015) 1-18.

[29] M. A. Mellal, E. Zio, A penalty guided stochastic fractal search approach for system reliability optimization, Reliability Engineering \& System Safety 152 (2016) 213-227.

[30] E. Çelik, Incorporation of stochastic fractal search algorithm into efficient design of pid controller for an automatic voltage regulator system, Neural Computing and Applications (2018) 1-12.

[31] J. Lin, Z.-J. Wang, Multi-area economic dispatch using an improved stochastic fractal search algorithm, Energy 166 (2019) 47-58.

[32] W. Gong, Z. Cai, Parameter extraction of solar cell models using repaired adaptive differential evolution, Solar Energy 94 (2013) 209-220.

[33] K. Yu, J. Liang, B. Qu, X. Chen, H. Wang, Parameters identification of photovoltaic models using an improved jaya optimization algorithm, Energy Conversion and Management 150 (2017) 742-753.

[34] D. H. Muhsen, A. B. Ghazali, T. Khatib, I. A. Abed, Parameters extraction of double diode photovoltaic modules model based on hybrid evolutionary algorithm, Energy conversion and management 105 (2015) $552-561$.

[35] X. Gao, Y. Cui, J. Hu, G. Xu, Z. Wang, J. Qu, H. Wang, Parameter extraction of solar cell models using improved shuffled complex evolution algorithm, Energy Conversion and Management 157 (2018) 460-479.

[36] S. Li, W. Gong, X. Yan, C. Hu, D. Bai, L. Wang, L. Gao, Parameter extraction of photovoltaic models using an improved teaching-learning-based optimization, Energy Conversion and Management 186 (2019) 293-305.

[37] J. J. Liang, A. K. Qin, P. N. Suganthan, S. Baskar, Comprehensive learning particle swarm optimizer for global optimization of multimodal functions, IEEE transactions on evolutionary computation 10 (2006) 281-295.

[38] X. Chen, H. Tianfield, C. Mei, W. Du, G. Liu, Biogeography-based learning particle swarm optimization, Soft Computing 21 (2017) 7519-7541.

[39] D. Karaboga, B. Basturk, A powerful and efficient algorithm for numerical function optimization: artificial bee colony (abc) algorithm, Journal of global optimization 39 (2007) 459-471. 
${ }^{*}$ Conflict of Interest Form

\section{Declaration of Interest Statement}

The authors have no conflict of interest, financial or otherwise. 
LaTeX Source Files
Click here to download LaTeX Source Files: Latex_elsarticle_pSFS4PV.rar

LaTeX Source Files
Click here to download LaTeX Source Files: Latex_elsarticle_pSFS4PV.rar

Click here to download LaTeX Source Files: Latex_elsarticle $p$ SFS4PVir

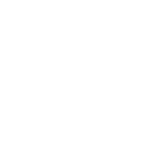

$\sqrt{3}$

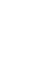
.

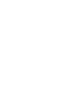
(1) (1) (1) (1) (1) . . . . . . . . . . . .

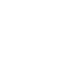

